\title{
Theoretical Comparative Energy Efficiency Analysis of Dual Axis Solar Tracking Systems
}

\author{
Aboubacarine Maiga \\ Ecole Normale d'Enseignement Technique et Professionnel (ENETP), Bamako, Mali \\ Email: crinemaiga@yahoo.fr
}

How to cite this paper: Maiga, A. (2021) Theoretical Comparative Energy Efficiency Analysis of Dual Axis Solar Tracking Systems. Energy and Power Engineering, 13, 448-482.

https://doi.org/10.4236/epe.2021.1312031

Received: November 23, 2021

Accepted: December 28, 2021

Published: December 31, 2021

Copyright $\odot 2021$ by author(s) and Scientific Research Publishing Inc. This work is licensed under the Creative Commons Attribution International License (CC BY 4.0).

http://creativecommons.org/licenses/by/4.0/

\begin{abstract}
This paper developed a theoretical model substantially based on the principle that only the normal component of solar radiation is actually converted into electrical energy. This theoretical model helped to predict minimum and maximum daily energy gain (compared to static PV system tilted with certain angle) when using dual axis PV solar tracking systems, at any given location on earth without prior experimental data. Based on equations derived from model, minimum and maximum energy gain is computed and summarized in tables of minimum and maximum. Furthermore, the model equations could be used to set up future experimental studies related to the matter.
\end{abstract}

\section{Keywords}

Solar Tracking Systems, Dual Axis, PV, Energy Efficiency, Optimum Tilt Angle

\section{Introduction}

The tracking mechanism is an electromechanical system that ensures solar radiation is always perpendicular to the surface of photovoltaic cells which maximize energy harnessing [1]. There are mainly two types of solar trackers based on the basis of their movement degrees of freedom [1]. These are single axis solar tracker and dual axis solar tracker [1]. Active, passive and chronological trackers are three of them [1]. The literature is rich in solar trackers design models developed and experimented [1]-[11].

[1] designed and built a hybrid solar tracking system whose energy efficiency was compared to continuous solar tracking systems (dual axis solar tracker) in terms of power consumption (due to actuators/motors); afterwards energy gain comparisons are performed between the four basic solar trackers: hybrid, continuous (dual), single axis solar trackers and static tilted PV systems. The experi- 
ments performed by [1] in Dhaka Bangladesh $\left(23^{\circ} 42^{\prime} 0^{\prime \prime} \mathrm{N} 90^{\circ} 22^{\prime} 30^{\prime \prime} \mathrm{E}\right)$ concluded that the average power gain of continuous tracking system over the static panel is $28.10 \%$ while [10] concluded that, after experimentation performed in South Africa, the additional energy gain achieved by dual axis solar tracking systems was about $37 \%$ compared to static PV systems.

The study performed by [5] in Eskisehir region $\left(39.78^{\circ} \mathrm{N} 30.52^{\circ} \mathrm{E}\right)$ concluded $33 \%$ energy gain of the experimental solar tracking system over the fixed PV panels. [3] focused on developing better LDR (Light Dependent Resistors) sensors which could increase the PV performance of sunlight tracking and concluded that energy efficiency could be increased up to $60 \%$ compared to static PV.

[4] presented the performance of dual axis solar tracker and static solar system with respect to clearness index in Malaysia and mentioned that the efficiency of DAST (Dual Axis Solar Tracker) over SSS (Static Solar System) varies from $24.91 \%$ at an overcast day to $82.12 \%$ at a clear day; while [11] calculated the efficiency of dual axis solar tracking systems over that of the static panel to be $81.68 \%$.

It appears that the aforementioned investigations concluded different numerical values regarding the energy gain achieved by dual axis solar tracking system over static PV system. The apparent discrepancies should be explained and understood through a theoretical framework: that is one of the motivations of this article. But the primarily motivation comes through the lack of methods or models that enable solar tracking systems efficiency experimentation results generalization, observed in the previous investigations related to the matter.

Therefore the major goal of this paper is to carry out results generalization of dual axis solar tracking systems energy efficiency over tilted static PV array depending on parameters that should be rigorously defined.

\section{Theoretical Assumptions}

Solar radiation is made up of three components: direct, diffuse and reflective. The uncertainty in predicted tilted irradiance is generally caused by the direct/diffuse separation [6]. In order to remove that uncertainty, we assume a clear sky conditions in which the major part of total irradiance is direct irradiance as concluded by many studies. Since the total irradiance is reduced to direct one, the total solar irradiance is represented by the single vector $\boldsymbol{I}_{k}$ (Figure 1). Intuitively we assume that the normal component of $\boldsymbol{I}_{k}$ is the only one which is actually converted into electrical power through PV cells array. Hence, the model assumptions are summarized as follows:

A1: Total solar irradiance is represented by vector $\boldsymbol{I}_{k}$. Magnitude of vector $\boldsymbol{I}_{k}$ is assumed to be the same in all directions.

A2: Normal component of $\boldsymbol{I}_{k}$ is the only one which is actually converted into electrical energy.

\section{Solar Noon, Best Slope Angle of a Tilted PV Cells Array}

The hour angle $\omega$ is zero at local noon (when the sun reaches its highest point 


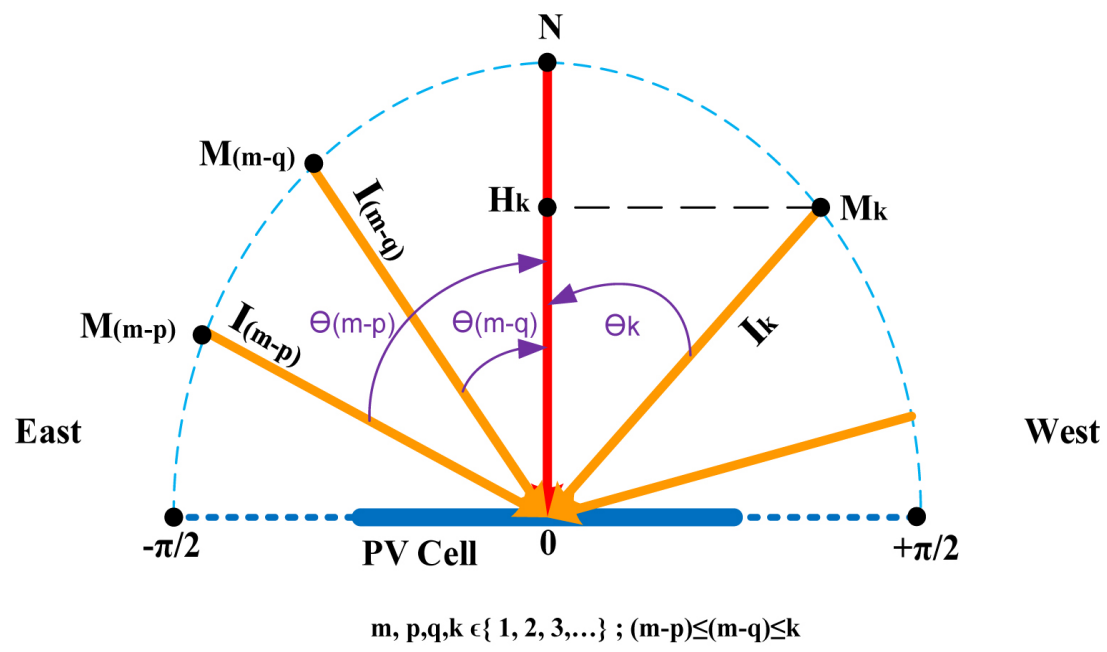

Figure $1^{1}$. Solar radiation normal components.

in the sky) [9]. On any day of the year, the most direct solar radiation at a given location occurs at solar noon [9]. Then, solar elevation angle $\alpha$ is defined as:

1) In the Northern hemisphere:

$$
\alpha=90^{\circ}-(\varphi-\delta)
$$

2) In the southern hemisphere:

$$
\alpha=90^{\circ}+(\varphi-\delta)
$$

where:

$\varphi$ is the latitude of location (See Appendix 1).

$\delta$ is the solar declination angle (See Appendix 1).

Let's consider PV cells array. When PV cells lie down in horizontal position, solar radiation reaches the PV cells under angle $\alpha$ (which is not necessary equal to $90^{\circ}$ ). It points out the need to make PV cells tilt with a certain angle $\beta$ called inclination angle (or tilt angle) so that the solar radiation is perpendicular to PV cells. Therefore, the best slope angle on a particular day of the year can be defined as the angle $\beta$ such that:

$$
\alpha+\beta=90^{\circ}
$$

Most of previous studies show that optimal fixed tilt angle of PV panels is south facing in the Northern Hemisphere and north facing in the Southern Hemisphere [12].

In this paper fixed (static) tilted PV cells are always assumed to be either north facing or south facing according the location in Northern Hemisphere or Southern Hemisphere

\section{PV Dual Axis Solar Tracking System versus Particular Case of Best-Slope-Angle Tilted Static PV Cells Array}

1) Let's consider a static tilted (with the best slope angle) PV cell in a particu${ }^{1} \mathrm{PV}$ cell in Figure 1 should be understood as a tilted PV plane. 
lar location of the earth and in a particular clear sky day of the year. In addition let's denote $T$ the length of the day (i.e., the time interval between the sunrise and the sunset). We could reasonably define a set of discrete points $\left\{M_{1}, M_{2}, \cdots, M_{m-p}, \cdots, M_{m-q}, \cdots, M_{k}, \cdots, M_{m}\right\}$ by which the sun apparent position goes through, as it moves from East to West.

The solar irradiance at time $t_{k}\left(0 \leq t_{k} \leq T\right)$ of the day is defined as:

$$
\boldsymbol{I}_{k}=\boldsymbol{M}_{k} \boldsymbol{O}
$$

2) If integer $m \rightarrow+\infty$, the discrete function $\theta_{k}$ can be expressed as continuous time function $\theta(t)$ :

$$
\theta(t)=\Omega t-\frac{\pi}{2}
$$

where:

- $\Omega=\frac{\pi}{T}$ defines the angular speed of sun apparent rotation;

- $0 \leq t \leq T$.

And the discrete normal component $\boldsymbol{I}_{k n}$ becomes a continuous component $I_{n}(t)$ :

$$
\boldsymbol{I}_{n}(t)=[\cos (\theta(t))] \cdot \mathbf{N O}
$$

The total solar energy (radiation) $R$ captured and actually converted into electrical energy by PV cells is evaluated:

$$
R=\int_{0}^{T}\left|\boldsymbol{I}_{n}(t)\right| \mathrm{d} t=\frac{2}{\pi}\left(I_{\max } * T\right)
$$

3) Dual axis solar tracking system ensures that solar radiation is normal at any time of the day. Therefore, the total solar energy (radiation) $R_{\max }$ captured by such a system is computed as:

$$
R_{\max }=I_{\text {max }} * T
$$

(7) and (8) yield:

$$
R_{\max }=\frac{\pi}{2} R \cong R+(0.57 * R)
$$

As the output electrical energy is in general considered to be proportional to the solar energy radiation, from (9) it can be stated that PV dual axis solar tracking system energy efficiency is about $60 \%$ higher than that of a best-slope-angle static tilted PV system.

\section{PV Dual Axis Solar Tracking System versus Static Tilted PV Cells Array}

Let's consider a static tilted PV cell located in Northern Hemisphere (Figure 2).

Furthermore, we consider an orthonormal rectangular coordinates system associated with the static tilted PV plane as shown in Figure 4.

The normal irradiance to $\mathrm{PV}$ plane is represented by $\mathrm{ON}$ and defined as: 


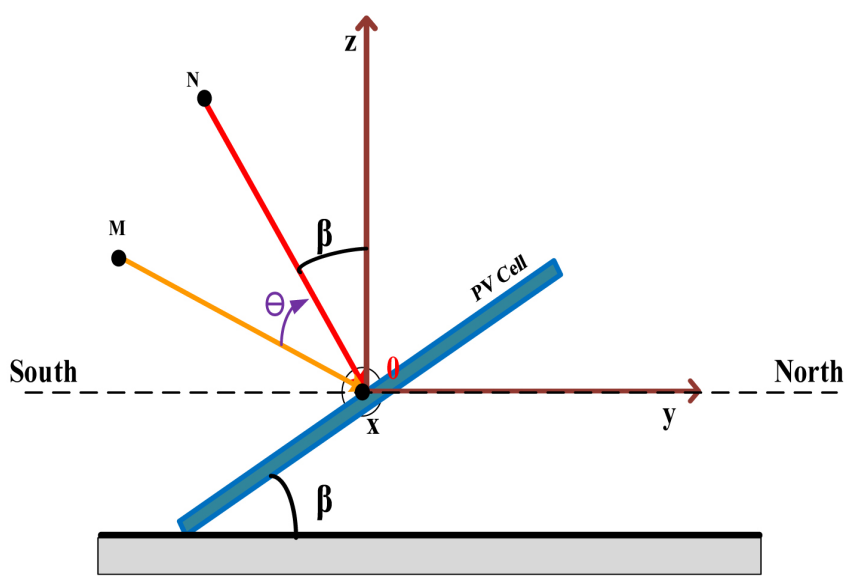

Figure 2. Axes of coordinates system tied to static tilted PV plane.

$$
\boldsymbol{O N}\left(\begin{array}{c}
0 \\
-I_{\max } \sin (\beta) \\
I_{\max } \cos (\beta)
\end{array}\right)
$$

where:

$\beta$ is the slope angle of $\mathrm{PV}$ cell plane.

$I_{\max }$ denotes the magnitude of $\boldsymbol{O N}$.

Solar apparent position, at any time, of the day is located by:

$$
\boldsymbol{O M}\left(\begin{array}{c}
I_{\max } \cos (\alpha) \sin (\gamma) \\
I_{\max } \cos (\alpha) \cos (\gamma) \\
I_{\max } \sin (\alpha)
\end{array}\right)
$$

where:

$\alpha$ denotes solar elevation (See Appendix 1);

$\gamma$ denotes solar azimuth angle (See Appendix 1).

Equation (11) is valid only if the horizontal axe of PV plane is rigorously aligned (parallel) with North-South direction.

Let's introduce angle $\lambda$ to take into account the fact that the horizontal axe of PV plane could be lightly either South-East and North-West oriented or South-West and North-East oriented (Figure 3).

(11) becomes then:

$$
\boldsymbol{O M}\left(\begin{array}{c}
I_{\max } \cos (\alpha) \sin (\gamma+\lambda) \\
I_{\max } \cos (\alpha) \cos (\gamma+\lambda) \\
I_{\max } \sin (\alpha)
\end{array}\right)
$$

Computing the scalar product of $O M$ and $O N$ it comes:

$$
\cos \theta=C_{1}+C_{2} \cos \omega+C_{3} \sin \omega
$$

where:

$$
\begin{aligned}
& C_{1}=\sin \delta(\cos \beta \sin \varphi-\sin \beta \cos \varphi \cos \lambda) \\
& C_{2}=\cos \delta(\cos \beta \cos \varphi+\sin \beta \sin \varphi \cos \lambda)
\end{aligned}
$$




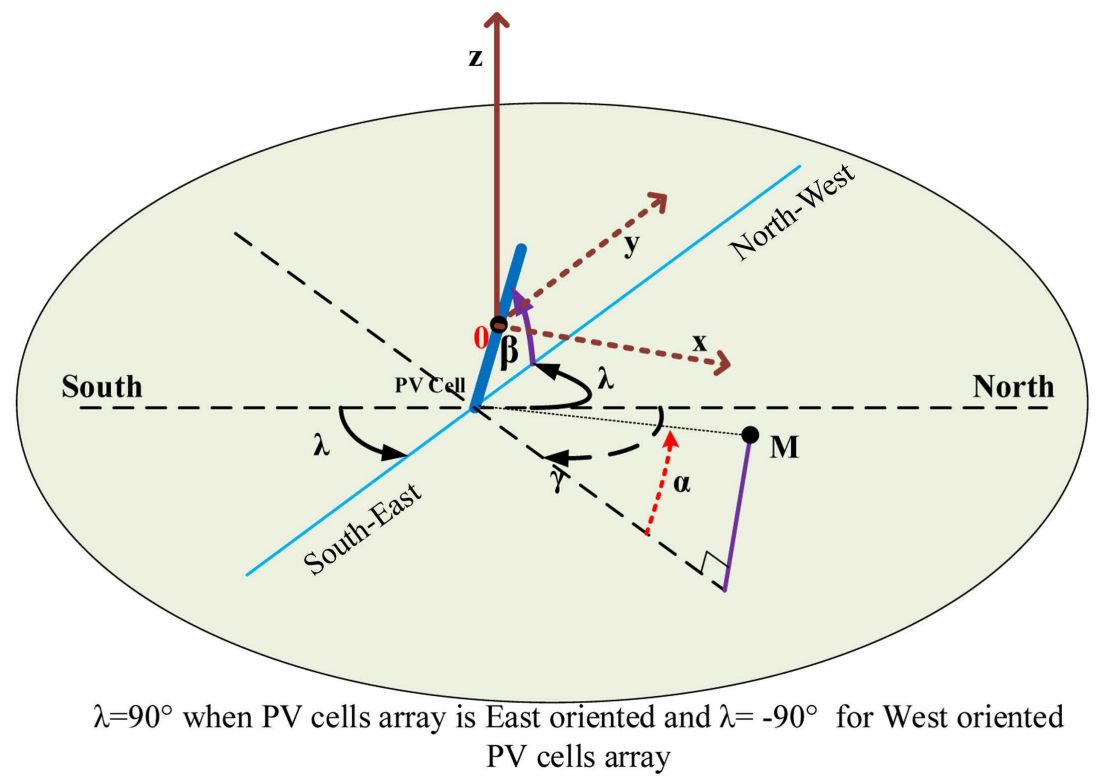

Figure 3. Deviation angle $(\lambda)$ of static tilted PV plane from north-south direction.

$$
C_{3}=\sin \beta \cos \delta \sin \lambda
$$

Let's denote $R$ the total solar energy captured by tilted PV plane with slope angle $\beta$ and azimuthal direction $(\pi-\lambda)$. Likewise (7):

$$
R=\int_{T_{\text {rise }}}^{T_{\text {set }}}\left|\boldsymbol{I}_{n}(t)\right| \mathrm{d} t=I_{\max } \int_{T_{\text {rise }}}^{T_{\text {set }}}|\cos \theta| \mathrm{d} t
$$

where:

$T_{\text {rise }}$ denotes time of sunrise (see Appendix 1);

$T_{\text {set }}$ denotes time of sunset (see Appendix 1).

From (13) and (17), derives:

$$
R_{\max }=R \frac{\pi T_{0}}{\left|C_{1} \pi T_{0}+12 C_{2} \sin \left(\frac{\pi T_{0}}{12}\right)\right|}
$$

Let' set:

$$
K=\frac{\pi T_{0}}{\left|C_{1} \pi T_{0}+12 C_{2} \sin \left(\frac{\pi T_{0}}{12}\right)\right|}
$$

(18) yields:

$$
R_{\max }=R+(K-1) R
$$

The amount of energy increase by a dual axis solar tracking system compared to static tilted PV system, in Northern Hemisphere, can be measured by:

$$
\eta(\varphi, \beta, n)=K(\varphi, \beta, n)-1
$$

In Southern Hemisphere variables $\varphi$ and $\beta$ should be replaced by their opposites, i.e. $-\varphi$ and $-\beta$.

${ }^{2}(18)$ is valid only if $\lambda$ is set to zero. 


\section{Efficiency Computations Results and Discussion}

The efficiency factor $\eta$ is computed using Matlab for a range of latitudes $\varphi$ (that are not in particular cases of latitudes for which there is no sunrise or sunset), slope angles $\beta$ of PV plane and for each day of the year.

Two tables are computed: the first (Tables A1-A6) gives minimum daily efficiencies (in percent) and the second (Tables A7-A12) gives maximum daily efficiencies, when latitude and inclination angles are set to be constant.

The plots of minimum efficiencies (Figure 4), as an illustration show, depending on inclination angle, that minimum efficiencies could be either increased or decreased.

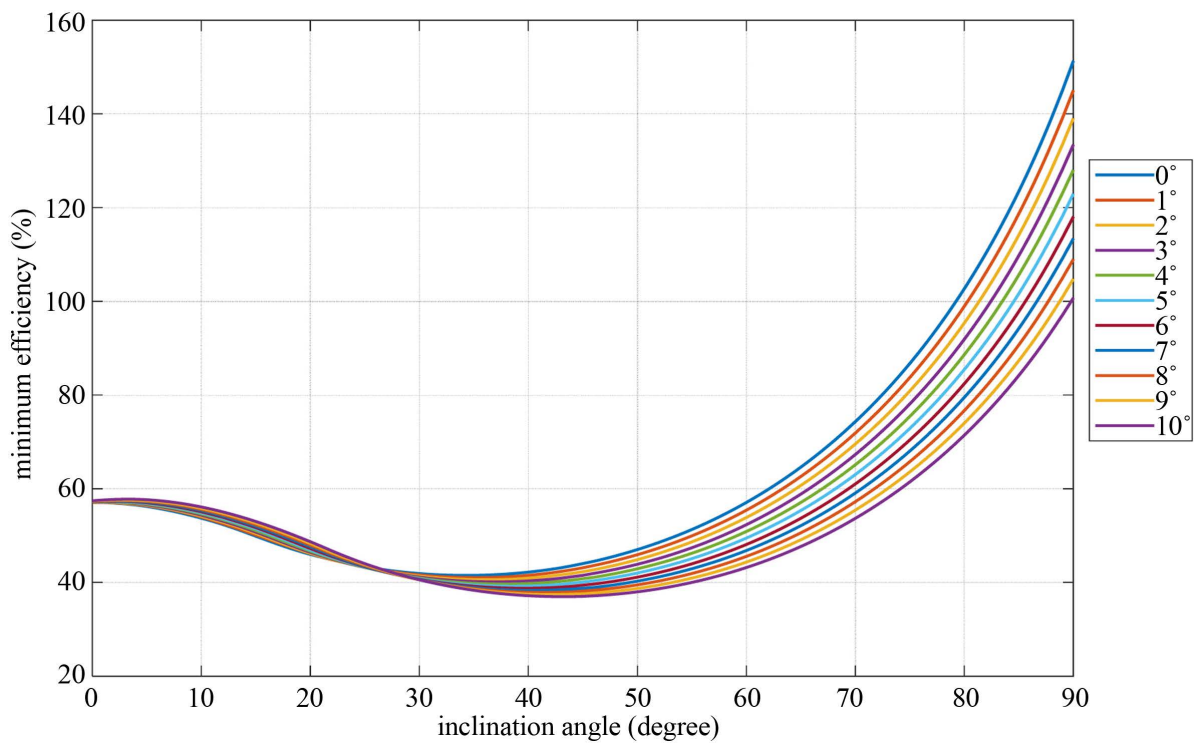

(a)
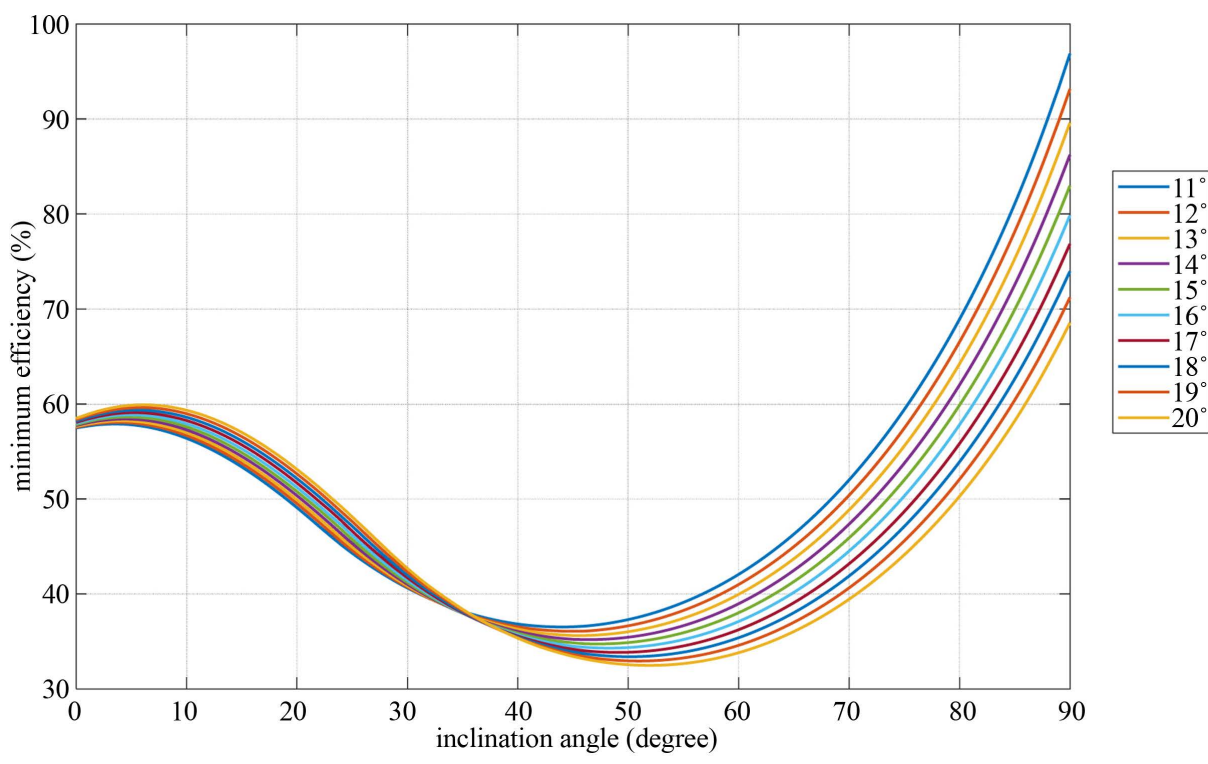

(b)

Figure 4. Minimum efficiencies plot (a) for latitudes $0^{\circ}$ to $10^{\circ}$; (b) for latitudes $11^{\circ}$ to $20^{\circ}$. 
The first major result which derives from minimum efficiencies table (Tables A1-A6) is that for any location on the earth there is particular slope angle which achieves the minimum of minimum efficiencies on a specific day.

Example 1: For latitude $40^{\circ}$, the minimum of minimum efficiencies is reached at slope angle $\beta=69^{\circ}$ on day number $n=355$.

The second major result which derives from minimum efficiencies table is a straightforward method for yearly optimum slope angle determination of static PV cells array: the optimum slope angle is the (minimum in case of more than one) slope angle for which minimum efficiency is closest to $\left(\frac{\pi}{2}-1\right)$, i.e. to $57 \%$.

Example 2: For latitude $0^{\circ}$ the slope angle which has the minimum efficiency closest to $57 \%$ is $0^{\circ}$, so $0^{\circ}$ is the yearly optimum angle, and for latitude $12^{\circ}$, the optimum slope angle would be $9^{\circ}$.

The experimental efficiencies held in previous works could not be accurately compared to data of Tables A1-A6 and Tables A7-A12 due to the fact that efficiency parameters (latitude, inclination angle of static PV cells array and day number), very often, are not explicitly and completely mentioned.

\section{Conclusions}

In this paper, energy gain factor (efficiency) of dual axis PV solar tracking systems compared to static (fixed) tilted PV arrays has been explicitly defined as a function of latitude, inclination angle, azimuthal deviation and day number, in a theoretical framework from theoretical model. Afterwards equation (21) computations using Matlab yielded Tables A1-A6 and Tables A7-A12 which, respectively, give minimum daily and maximum daily efficiencies for a wide range of latitudes and inclination angles.

The work carried out also gives an important theoretical framework necessary for precise experimental investigations so that Tables A1-A6 and Tables A7-A12 data could be compared to experimental data. It also suggested a straightforward method for optimum slope angle determination of static PV cells array. That method should be fully investigated and assessed in future works related to the matter. Another important research direction could be to analyze how meteorological conditions (cloudy or clear sky conditions) could alter the results of this paper.

\section{Acknowledgements}

The author thanks Dr. Maliki Guindo from National School of Engineers (ENI-ABT) for having revised the content of this article. Thanks to the Academic Staff of ENETP and National Higher Education Ministry for their support to the publication of this paper. Special thanks to Dr. Hamadoun Toure for his mentorship.

\section{Conflicts of Interest}

The author declares no conflicts of interest regarding the publication of this paper. 


\section{References}

[1] Ferdaus, R.A., Mohammed, M.A., Rahman, S., Salehin, S. and Mannan, M.A. (2014) Energy Efficient Hybrid Dual Axis Solar Tracking System. Journal of Renewable Energy, 2014, Article ID: 629717. https://doi.org/10.1155/2014/629717

[2] Allw, A.S. and Shallal, I.H. (2020) Evaluation of Photovoltaic Solar Power of a Dual-Axis Solar Tracking System. Journal of Southwest Jiaotong University, 55, 9 p. https://doi.org/10.35741/issn.0258-2724.55.1.52

[3] bin Ibrahim, A.I., binti Abdul Rahman, F.D. and bin Rohaizat, M. (2018) Dual Axes Solar Tracker. International Journal of Electrical and Computer Engineering (IJECE), 8, 1887-1892. https://doi.org/10.11591/ijece.v8i3.pp1887-1892

[4] Lee, J.F., Rahim, N.Abd. and. Al-Turki, Y.A. (2013) Performance of Dual-Axis Solar Tracker versus Static Solar System by Segmented Clearness Index in Malaysia. International Journal of Photoenergy, 2013, Article ID: 820714. https://doi.org/10.1155/2013/820714

[5] Filik, T. and Başaran Filik, Ü. (2017) Efficiency Analysis of the Solar Tracking PV Systems in ESKIŞEHIR Region. Anadolu University Journal of Science and Technology A-Applied Sciences and Engineering, 18, 209-217. https://doi.org/10.18038/aubtda.267116

[6] Gueymard, C.A. (2008) From Global Horizontal to Global Tilted Irradiance: How Accurate Are Solar Energy Engineering Predictions in Practice? Solar Conf., San Diego, American Solar Energy Society.

[7] Hussein, M.T. and Albarqouni, S.N. (2010) Enhanced Model of One Axis-Two Positions Manual Tracking Photovoltaic Panels for Lighting Projects in Palestine. Proceedings of the Second IASTED International Conference, Solar Energy, 15-17 July 2010, Alberta, Canada, 937-943.

[8] Gilman, P. (2015) SAM Model Technical Reference. National Renewable Energy Laboratory (NREL), Technical Report.

[9] Introduction to Solar Geometry. http://mypages.iit.edu

[10] Vermaak, H.J. (2014) Technico-Economic Analysis of Solar Tracking Systems in South Africa. Energy Procedia, 61, 2435-2438.

https://doi.org/10.1016/j.egypro.2014.12.018

[11] Dhanabal, R., Bharathi, V., Ranjitha, R., Ponni, A., Deepthi, S. and Mageshkannan, P. (2013) Comparison of Efficiencies of Solar Tracker Systems with Static Panel Single Axis Tracking System and Dual Axis Tracking System with Fixed Mount. Proceedings of the Second IASTED International Journal of Engineering and Technology (IJET), 5, No. 2.

[12] Rouholamini, A., Pourgharibshahi, H., Fadaeinedjad, R. and Moschopoulos, G. (2013) Optimal Tilt Angle Determination of Photovoltaic Panels And Comparing of Their Mathematical Model Predictions to Experimental Data in Kerman. 2013 26th IEEE Canadian Conference of Electrical and Computer Engineering (CCECE). Regina, 5-8 May 2013, 1-4. https://doi.org/10.1109/CCECE.2013.6567674 


\section{Appendix}

The earth follows a complex motion that consists of the daily motion and the annual motion. The daily motion causes the sun to appear in the east to west direction over the earth whereas the annual motion causes the sun to tilt at a particular angle while moving along east to west direction [1]. Declination angle $\delta$ (Figure A1 [7]) accounts for annual motion; azimuth angle $\gamma$ (Figure A2 [8]) and elevation (or altitude) angle $\alpha$ (Figure A2 [8]) characterize the daily motion.

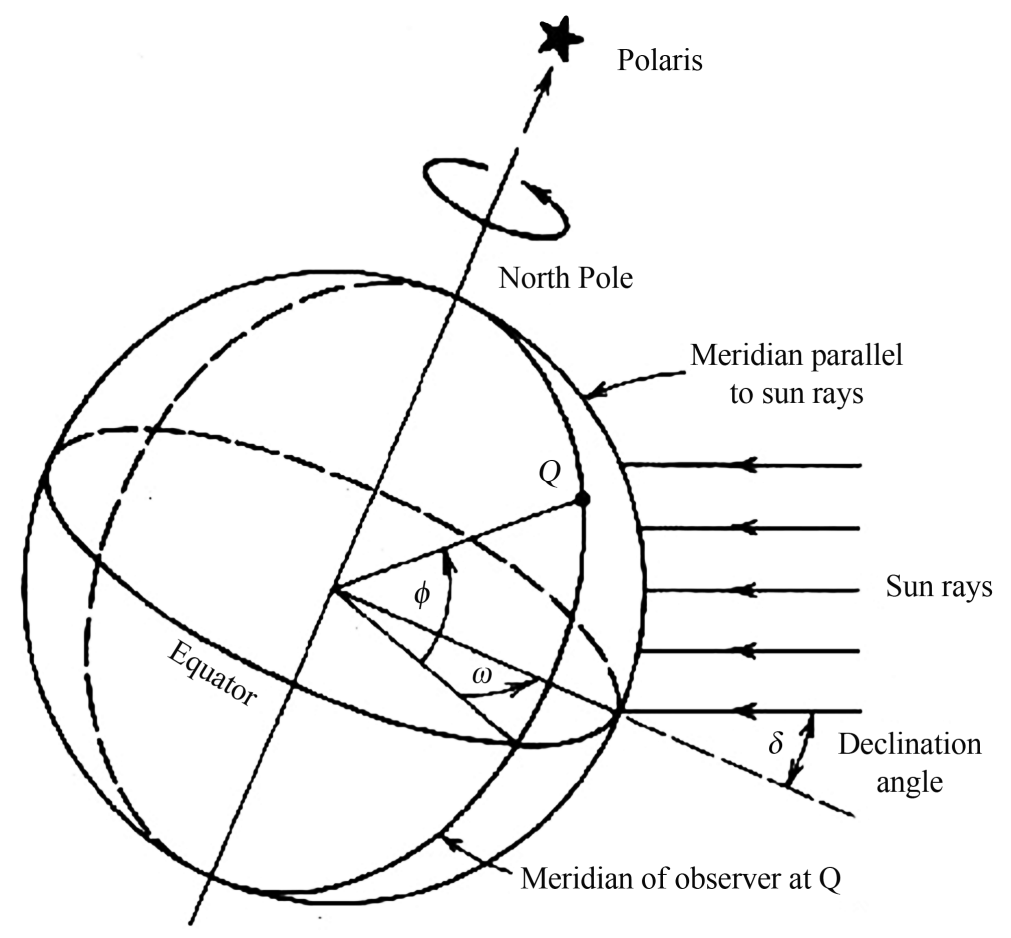

Figure A1. Declination angle $(\delta)$, Latitude $(\phi)$ and Hour angle $(\omega)$.

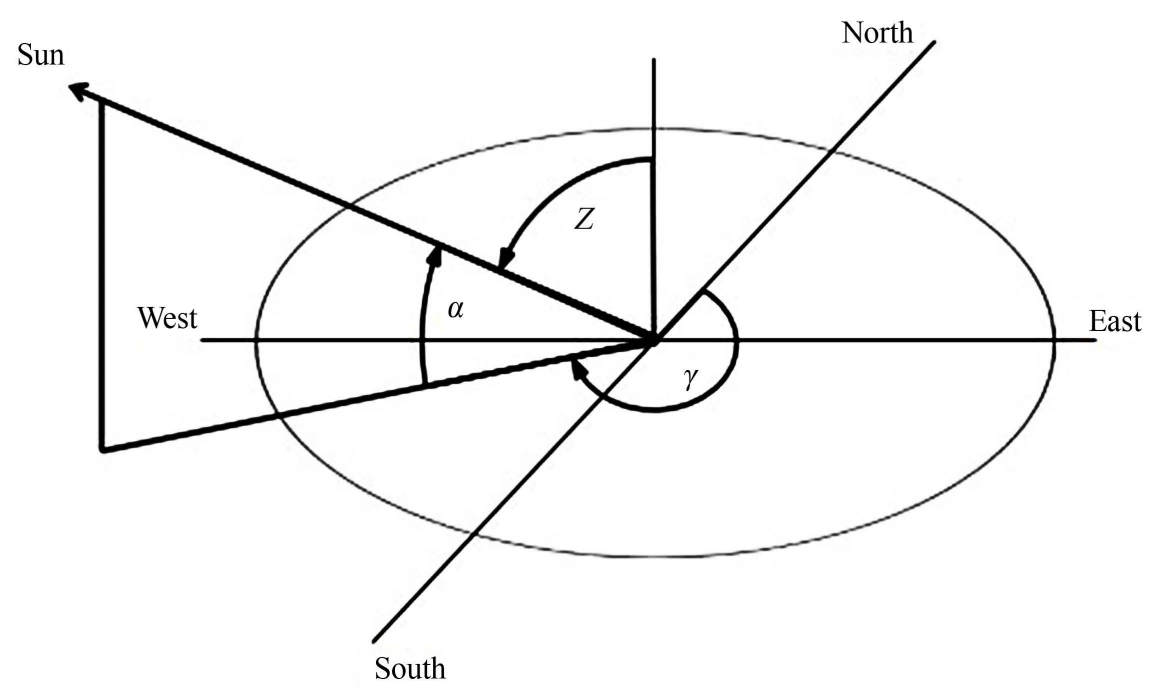

Figure A2. Solar Azimuth angle ( $\gamma)$, Elevation angle $(\alpha)$ and Zenith angle $(Z)$. 
1) Sun declination angle $\delta$ is defined to be that angle made between a ray of the sun, when extended to the center of the earth and the equatorial plane [9].

$$
\delta=23.45 \sin \left[360\left(\frac{284+n}{365}\right)\right]
$$

where:

$\delta:$ Declination angle in degrees;

$n$ : day number ( $n=1$ at the first of January).

2) Solar elevation (altitude) angle $\alpha$ (Figure A2) is the angle between the projection of the sun's rays on the horizontal plane and the direction on the sun's rays [7].

From [9]:

$$
\alpha=\sin ^{-1}(\sin \delta \sin \varphi+\cos \delta \cos \varphi \cos \omega)
$$

where:

$$
\begin{aligned}
& \delta \text { : Declination angle; } \\
& \varphi \text { : Observer's latitude; } \\
& \omega \text { : hour angle. }
\end{aligned}
$$

3) Hour angle $(\omega)$ is the angular displacement from east to west of the local meridian due to rotation of the earth on its axis at $15^{\circ}$ per hour [7]:

$$
\omega=15(t-12)
$$

4) Latitude $\varphi$ (Figure A1) of a location on the earth is the angle between the line joining that location to the center of the earth and the equatorial plane [9].

5) Zenith angle $Z$ (Figure A2) is the complement of altitude angle [7]:

$$
Z=90^{\circ}-\alpha
$$

6) Azimuth angle $\gamma$ is the local angle between the direction of due north and that of the perpendicular projection of the sun down onto the horizon line measured clockwise [9].

The following relation relates the sun azimuth angle and its elevation angle:

$$
\cos \alpha \cos \gamma=\sin \delta \cos \varphi-\cos \delta \sin \varphi \cos \omega
$$

It can also be shown that:

$$
\cos \alpha \sin \gamma=\cos \delta \sin \omega
$$

7) Latitude $\varphi$ (denoted by $\phi$ on Figure A1) of a location on the earth is the angle between the line joining that location to the center of the earth and the equatorial plane [9].

8) Sunrise and sunset time equations are defined as follows:

$$
\begin{gathered}
T_{\text {rise }}=12-T_{0} \\
T_{\text {set }}=12+T_{0}
\end{gathered}
$$

With

$$
T_{0}=\frac{12}{\pi} \cos ^{-1}(-\tan \varphi \tan \delta)
$$


Table A1. Minimum daily efficiencies (in percent) for latitudes $\left[0^{\circ}, 65^{\circ}\right]$; slope angles $\left[0^{\circ}, 15^{\circ}\right]$.

\begin{tabular}{|c|c|c|c|c|c|c|c|c|c|c|c|c|c|c|c|c|}
\hline & 0 & 1 & 2 & 3 & 4 & 5 & 6 & 7 & 8 & 9 & 10 & 1 & 12 & 13 & 14 & 5 \\
\hline 0 & 3 & 7.04 & 5.94 & 7 & & .21 & & & & & & & & & 4 & 39 \\
\hline 1 & & .07 & & & 56.61 & 56.32 & & & 7 & 54.52 & 2 & 7 & 6 & 1 & 1 & 0.17 \\
\hline 2 & 9 & 57.10 & 7.04 & L & 56.71 & 5 & 56.11 & 1 & 5 & 2 & 4.14 & .50 & .81 & 2.07 & 1.28 & 0.46 \\
\hline 3 & 1 & 57.14 & 10 & 9 & 5 & 5 & 5 & 7 & .43 & 2 & 4.36 & 4 & 6 & 4 & 57 & 50.75 \\
\hline 4 & 13 & 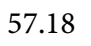 & 1 & 3 & 3 & 5 & 1 & 5 & 2 & 4 & 9 & 8 & & 1 & 6 & 51.05 \\
\hline 5 & 16 & 57.2 & 24 & 18 & 57.0 & 5 & 56.56 & 56.23 & 55.82 & 5 & 54.82 & 4.24 & 53.59 & 2.90 & 2.15 & 51.36 \\
\hline 6 & 19 & .29 & 57.3 & - & 5 & 5 & 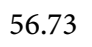 & 1 & 56.03 & 8 & 6 & 9 & 7 & 9 & 15 & 51.67 \\
\hline 7 & 23 & 7.35 & 57.4 & 57.38 & 57.29 & 57.1 & 56.90 & 56.60 & 5 & 5 & 55.31 & 54.76 & 54. & 8 & 2.76 & 52.00 \\
\hline 8 & 28 & 57.42 & 57.50 & .50 & 5 & 5 & 5 & 5 & 6 & 5 & 7 & 3 & & 8 & 8 & 52.33 \\
\hline 9 & .33 & 7.50 & 57.60 & 62 & 57.5 & 57.46 & ' & 1 & 56.68 & - & 55.83 & 55.31 & & & & 52.66 \\
\hline 10 & 39 & 5 & 57.7 & 5 & 57.7 & 57 & 57. & 57. & 5 & & 56 & 0 & & & 4 & 53.01 \\
\hline 11 & 46 & 57.68 & 57.82 & 9 & 3 & 5 & 57 & & & & 8 & & & & & 53.36 \\
\hline 12 & 4 & . & s & & 58 & 58 & 57 & 3 & 11 & & 7 & 0 & & & & 53.73 \\
\hline 13 & 57.62 & 788 & 07 & 19 & 58.23 & 58.20 & 58 & 5 & 57.67 & 57 & 56 & 56 & 0 & & 9 & 54.10 \\
\hline 14 & 72 & 8.00 & & & 8.41 & & & & & & & & & & & 54.48 \\
\hline 15 & 57.82 & 0.12 & 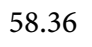 & 52 & 61 & 2 & 5 & & 22 & & 9 & & & & & 37 \\
\hline 16 & 93 & 26,5 & 5 & 70 & 20 & 5 & 58 & 5 & 5 & & 57 & 2 & 6 & 2 & 3 & 55.27 \\
\hline 17 & 05 & 0 & 5 & & & & & & & & & & & & & 55.69 \\
\hline 18 & 18 & 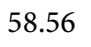 & & & & & & & & & & & & & & \\
\hline 19 & 32 & 872 & 59.05 & 0 & 59.48 & & 5 & & 5 & & & 2 & & & 7 & \\
\hline 20 & 48 & 90 & & & & & & & & & & & & & & 57.00 \\
\hline 21 & & 59.09 & & & & & & & & & & & & & & 57.46 \\
\hline 22 & 3.82 & & & & & & & & & & & 4 & & & & 57.94 \\
\hline 23 & 02 & 51 & 93 & 28 & 5 & 6 & & & 7 & & & 88 & 2 & 0 & .00 & 58.44 \\
\hline 24 & 39.22 & 39.14 & & & & & & & & & & & & & & 58.95 \\
\hline 25 & 59.45 & & & & & & & & & & & & & & & 5 \\
\hline 26 & 59.69 & 60.26 & 60.75 & 17 & 2 & 6 & 7 & 8 & 0 & & 0 & 68 & 8 & 0 & .55 & 60.02 \\
\hline 27 & 97 & 60.55 & 6 & 51 & 8 & 17 & 8 & 0 & 5 & & 9 & 9 & 0 & 4 & 10 & 60.58 \\
\hline 28 & 0 & 6 & 61.40 & 6 & 6 & 6 & 0 & & 2 & 0 & 0 & 72 & 5 & 0 & 67 & 61.17 \\
\hline 29 & 68 & 61.20 & 01. & 4 & 6 & 62.99 & 5 & & 1 & & 3 & 7 & 1 & 8 & 27 & 61.77 \\
\hline 30 & .11 & 01.00 & 62.13 & 55 & 6 & 0 & 2 & 2 & 3 & 5 & 9 & 34 & 50 & 9 & 89 & 62.4 \\
\hline 31 & 61.60 & 62.05 & 02.50 & 63.08 & 63.54 & 03.92 & 64.22 & 64.44 & 64.57 & 64.61 & 64.57 & 64.44 & 64.22 & 63.92 & 63.53 & 63.06 \\
\hline 32 & 62.15 & 62.56 & 63.02 & 63.54 & 64.02 & 64.43 & 64.75 & 64.99 & 65.14 & 65.20 & 65.18 & 65.06 & 64.86 & 64.57 & 64.20 & 63.74 \\
\hline
\end{tabular}




\section{Continued}

\begin{tabular}{|c|c|c|c|c|c|c|c|c|c|c|c|c|c|c|c|c|}
\hline 33 & 62.75 & 63.13 & 63.56 & 64.03 & 64.53 & 64.96 & 65.31 & 65.57 & 65.74 & 65.82 & 65.82 & 65.72 & 65.54 & 65.26 & 64.90 & 64.45 \\
\hline 34 & 63.42 & 63.76 & 64.15 & 64.59 & 65.08 & 65.53 & 65.90 & 66.18 & 66.37 & 66.48 & 66.49 & 66.41 & 6.24 & 65.98 & 65.63 & 65.19 \\
\hline 35 & 64.14 & 64.45 & 64.80 & 65.21 & 65.67 & 66.14 & 66.53 & 66.83 & 7.04 & 67.16 & 67.19 & 7.13 & 6.98 & 66.73 & 66.39 & 65.96 \\
\hline 36 & 64.93 & 65.20 & 65.52 & 65.89 & 66.32 & 66.79 & 67.19 & 67.52 & 67.75 & 67.89 & 67.94 & 67.89 & 7.75 & 67.52 & 67.19 & 66.77 \\
\hline 37 & 65.78 & 66.02 & 66.30 & 66.64 & 67.03 & 67.48 & 67.91 & 68.25 & 68.50 & 68.66 & 68.72 & 68.69 & 8.57 & 68.34 & 68.03 & 67.62 \\
\hline 38 & 66.70 & 66.90 & 67.15 & 67.46 & 67.82 & 68.23 & 68.66 & 69.02 & 69.29 & 69.47 & 69.55 & 69.54 & 69.42 & 69.21 & 68.91 & 68.51 \\
\hline 39 & 67.68 & 67.85 & 68.07 & 68.35 & 68.67 & 69.05 & 69.47 & 69.85 & 70.14 & 70.33 & 70.43 & 70.43 & 70.33 & 70.13 & 69.83 & 69.44 \\
\hline 40 & 68.74 & 68.88 & 69.07 & 69.31 & 69.60 & 69.94 & 70.34 & 70.73 & 71.04 & 71.24 & 71.36 & 71.37 & 71.28 & 71.09 & 70.80 & 70.41 \\
\hline 41 & 69.87 & 69.97 & 70.13 & 70.34 & 70.60 & 70.91 & 71.28 & 71.68 & 71.99 & 72.22 & 72.34 & 72.36 & 72.29 & 72.11 & 71.83 & 71.44 \\
\hline 42 & 71.08 & 71.15 & 71.27 & 71.45 & 71.68 & 71.97 & 72.30 & 72.69 & 73.02 & 73.25 & 73.39 & 73.42 & 73.35 & 73.18 & 72.90 & 72.52 \\
\hline 43 & 72.36 & 72.40 & 72.50 & 72.65 & 72.85 & 73.10 & 73.41 & 73.77 & 74.11 & 74.36 & 74.50 & 74.55 & 74.48 & 74.32 & 74.05 & 3.67 \\
\hline 44 & 73.73 & 73.74 & 73.81 & 73.93 & 74.10 & 74.32 & 74.60 & 74.94 & 75.28 & 75.54 & 75.69 & 75.74 & 75.69 & 75.52 & 75.25 & 74.87 \\
\hline 45 & 75.19 & 75.17 & 75.21 & 75.30 & 75.44 & 75.64 & 75.89 & 76.20 & 76.54 & 76.80 & 76.96 & 77.01 & 76.96 & 76.80 & 76.53 & 76.15 \\
\hline 46 & 76.73 & 76.69 & 76.70 & 76.76 & 76.88 & 77.05 & 77.28 & 77.56 & 77.88 & 78.15 & 78.31 & 78.37 & 78.32 & 78.16 & 77.89 & 77.50 \\
\hline 47 & 78.38 & 78.31 & 78.29 & 78.33 & 78.42 & 78.57 & 78.77 & 79.02 & 79.33 & 79.60 & 79.76 & 79.82 & 79.77 & 79.61 & 79.33 & 78.94 \\
\hline 48 & 80.12 & 80.03 & 79.99 & 80.00 & 80.07 & 80.19 & 80.37 & 80.60 & 80.89 & 81.16 & 81.32 & 81.37 & 81.32 & 81.15 & 80.86 & 80.46 \\
\hline 49 & 81.98 & 81.86 & 81.80 & 81.79 & 81.83 & 81.93 & 82.09 & 82.31 & 82.57 & 82.83 & 82.99 & 83.03 & 82.97 & 82.79 & 82.49 & 82.08 \\
\hline 50 & 83.95 & 83.81 & 83.72 & 83.69 & 83.72 & 83.80 & 83.94 & 84.14 & 84.39 & 84.63 & 84.78 & 84.82 & 84.74 & 84.55 & 84.24 & 83.80 \\
\hline 51 & 86.05 & 85.89 & 85.78 & 85.73 & 85.74 & 85.81 & 85.93 & 86.11 & 86.35 & 86.58 & 86.71 & 86.73 & 86.64 & 86.43 & 86.10 & 85.64 \\
\hline 52 & 88.28 & 88.10 & 87.98 & 87.92 & 87.91 & 87.96 & 88.07 & 88.24 & 88.46 & 88.67 & 88.79 & 88.79 & 88.67 & 88.44 & 88.08 & 87.60 \\
\hline 53 & 90.65 & 90.46 & 90.33 & 90.25 & 90.24 & 90.28 & 90.38 & 90.53 & 90.75 & 90.94 & 91.03 & 91.00 & 90.86 & 90.60 & 90.21 & 89.70 \\
\hline 54 & 93.19 & 92.99 & 92.84 & 92.76 & 92.74 & 92.77 & 92.86 & 93.02 & 93.22 & 93.38 & 93.44 & 93.39 & 93.21 & 92.92 & 92.50 & 91.94 \\
\hline 55 & 95.90 & 95.69 & 95.54 & 95.46 & 95.43 & 95.46 & 95.56 & 95.71 & 95.91 & 96.03 & 96.05 & 95.96 & 95.75 & 95.41 & 94.94 & 94.35 \\
\hline 56 & 98.80 & 98.59 & 98.45 & 98.36 & 98.34 & 98.38 & 98.48 & 98.63 & 98.81 & 98.89 & 87 & 98.73 & 98.47 & 98.09 & 97.57 & 96.92 \\
\hline 57 & 101.91 & 101.72 & 101.58 & 101.51 & 101.50 & 101.55 & 101.66 & 101.83 & 101.96 & 101.99 & 101.92 & 101.73 & 101.42 & 100.97 & 100.40 & 99.69 \\
\hline 58 & 105.28 & 105.10 & 104.98 & 104.93 & 104.94 & 105.01 & 105.14 & 105.30 & 105.38 & 105.35 & 105.22 & 104.97 & 104.59 & 104.09 & 103.44 & 102.66 \\
\hline 59 & 108.93 & 108.78 & 108.69 & 108.67 & 108.71 & 108.81 & 108.97 & 109.08 & 109.09 & 109.00 & 108.80 & 108.47 & 108.02 & 107.44 & 106.72 & 105.85 \\
\hline 60 & 112.91 & 112.80 & 112.76 & 112.79 & 112.87 & 113.02 & 113.15 & 113.18 & 113.12 & 112.95 & 112.67 & 112.27 & 111.73 & 111.06 & 110.25 & 109.28 \\
\hline 61 & 117.31 & 117.26 & 117.28 & 117.37 & 117.52 & 117.65 & 117.69 & 117.65 & 117.50 & 117.25 & 116.88 & 116.38 & 115.75 & 114.98 & 114.06 & 112.98 \\
\hline 62 & 122.21 & 122.26 & 122.37 & 122.54 & 122.65 & 122.69 & 122.65 & 122.51 & 122.27 & 121.92 & 121.45 & 120.84 & 120.10 & 119.21 & 118.17 & 116.97 \\
\hline 63 & 127.81 & 127.98 & 128.13 & 128.23 & 128.25 & 128.19 & 128.05 & 127.81 & 127.46 & 127.00 & 126.41 & 125.68 & 124.81 & 123.79 & 122.61 & 121.27 \\
\hline 64 & 134.27 & 134.39 & 134.45 & 134.45 & 134.37 & 134.21 & 133.96 & 133.60 & 133.13 & 132.54 & 131.81 & 130.95 & 129.93 & 128.76 & 127.42 & 125.91 \\
\hline 65 & 141.40 & 141.42 & 141.38 & 141.27 & 141.08 & 140.80 & 140.42 & 139.93 & 139.32 & 138.58 & 137.70 & 136.67 & 135.49 & 134.14 & 132.62 & 130.92 \\
\hline
\end{tabular}


Table A2. Minimum daily efficiencies (in percent) for latitudes $\left[0^{\circ}, 65^{\circ}\right]$; slope angles $\left[16^{\circ}, 30^{\circ}\right]$.

\begin{tabular}{|c|c|c|c|c|c|c|c|c|c|c|c|c|c|c|c|}
\hline & 16 & 17 & 18 & 19 & 20 & 21 & 22 & 23 & 24 & 25 & 26 & 27 & 28 & 29 & 30 \\
\hline 0 & 49.01 & 48.18 & 47.40 & 46.67 & 46.00 & 45.38 & 44.80 & 44.28 & 43.80 & 43.37 & 42.98 & 42.64 & 42.35 & 42.10 & 41.89 \\
\hline 1 & 49.29 & 48.41 & 47.59 & 46.82 & 46.11 & 45.44 & 44.83 & 44.26 & 43.74 & 43.27 & 42.85 & 42.47 & 42.14 & 41.85 & 41.60 \\
\hline 2 & 49.59 & 48.68 & 47.82 & 47.01 & 46.25 & 45.54 & 44.88 & 44.28 & 43.72 & 43.21 & 42.75 & 42.33 & 41.96 & 41.63 & 41.35 \\
\hline 3 & 49.89 & 49.00 & 48.09 & 47.23 & 46.43 & 45.68 & 44.98 & 44.33 & 43.74 & 43.19 & 42.68 & 42.23 & 41.82 & 41.45 & 41.13 \\
\hline 4 & 50.20 & 49.32 & 48.40 & 47.50 & 46.65 & 45.86 & 45.12 & 44.43 & 43.79 & 43.20 & 42.65 & 42.16 & 41.71 & 41.31 & 40.95 \\
\hline 5 & 50.52 & 49.65 & 48.74 & 47.80 & 46.91 & 46.07 & 45.29 & 44.56 & 43.88 & 43.24 & 42.66 & 42.13 & 41.64 & 41.20 & 40.80 \\
\hline 6 & 50.85 & 49.99 & 49.08 & 48.15 & 47.21 & 46.33 & 45.50 & 44.72 & 44.00 & 43.33 & 42.70 & 42.13 & 41.60 & 41.12 & 40.69 \\
\hline 7 & 51.18 & 50.33 & 49.44 & 48.51 & 47.55 & 46.62 & 45.75 & 44.93 & 44.16 & 43.45 & 42.78 & 42.17 & 41.60 & 41.08 & 40.61 \\
\hline 8 & 51.52 & 50.68 & 49.80 & 48.88 & 47.92 & 46.95 & 46.04 & 45.17 & 44.36 & 43.61 & 42.90 & 42.24 & 41.64 & 41.08 & 40.56 \\
\hline 9 & 51.87 & 51.04 & 50.16 & 49.25 & 48.31 & 47.33 & 46.36 & 45.45 & 44.60 & 43.80 & 43.05 & 42.35 & 41.70 & 41.11 & 40.55 \\
\hline 10 & 52.23 & 51.41 & 50.54 & 49.64 & 48.70 & 47.72 & 46.73 & 45.77 & 44.87 & 44.03 & 43.24 & 42.50 & 41.81 & 41.17 & 40.58 \\
\hline 11 & 52.60 & 51.78 & 50.93 & 50.03 & 49.09 & 48.13 & 47.13 & 46.13 & 45.19 & 44.30 & 43.46 & 42.68 & 41.95 & 41.27 & 40.63 \\
\hline 12 & 52.97 & 52.17 & 51.32 & 50.43 & 49.50 & 48.54 & 47.55 & 46.53 & 45.54 & 44.60 & 43.72 & 42.90 & 42.12 & 41.40 & 40.73 \\
\hline 13 & 53.35 & 52.56 & 51.72 & 50.84 & 49.92 & 48.96 & 47.97 & 46.96 & 45.93 & 44.94 & 44.02 & 43.15 & 42.33 & 41.57 & 40.85 \\
\hline 14 & 53.75 & 52.96 & 52.13 & 51.26 & 50.34 & 49.39 & 48.41 & 47.39 & 46.36 & 45.33 & 44.35 & 43.44 & 42.58 & 41.77 & 41.01 \\
\hline 15 & 54.15 & 53.38 & 52.55 & 51.69 & 50.78 & 49.83 & 48.85 & 47.84 & 46.80 & 45.74 & 44.72 & 43.76 & 42.86 & 42.00 & 41.20 \\
\hline 16 & 54.56 & 53.80 & 52.99 & 52.13 & 51.22 & 50.28 & 49.30 & 48.30 & 47.26 & 46.20 & 45.13 & 44.12 & 43.17 & 42.28 & 41.43 \\
\hline 17 & 54.99 & 54.23 & 53.43 & 52.58 & 51.68 & 50.74 & 49.77 & 48.76 & 47.73 & 46.66 & 45.58 & 44.52 & 43.52 & 42.58 & 41.69 \\
\hline 18 & 55.42 & 54.68 & 53.88 & 53.04 & 52.15 & 51.21 & 50.24 & 49.24 & 48.20 & 47.14 & 46.06 & 44.96 & 43.91 & 42.92 & 41.99 \\
\hline 19 & 55.87 & 55.14 & 54.35 & 53.51 & 52.63 & 51.70 & 50.73 & 49.73 & 48.69 & 47.63 & 46.54 & 45.43 & 44.34 & 43.30 & 42.32 \\
\hline 20 & 56.33 & 55.61 & 54.83 & 54.00 & 53.12 & 52.19 & 51.23 & 50.23 & 49.19 & 48.13 & 47.04 & 45.93 & 44.80 & 43.72 & 42.69 \\
\hline 21 & 56.81 & 56.09 & 55.32 & 54.50 & 53.62 & 52.70 & 51.74 & 50.74 & 49.70 & 48.64 & 47.55 & 46.43 & 45.30 & 44.17 & 43.09 \\
\hline 22 & 57.30 & 56.59 & 55.83 & 55.01 & 54.14 & 53.22 & 52.26 & 51.26 & 50.23 & 49.16 & 48.07 & 46.95 & 45.81 & 44.65 & 43.53 \\
\hline 23 & 57.80 & 57.11 & 56.35 & 55.54 & 54.67 & 53.76 & 52.80 & 51.80 & 50.77 & 49.70 & 48.60 & 47.48 & 46.33 & 45.17 & 44.00 \\
\hline 24 & 58.32 & 57.64 & 56.89 & 56.08 & 55.22 & 54.31 & 53.36 & 52.36 & 51.32 & 50.25 & 49.15 & 48.02 & 46.87 & 45.70 & 44.51 \\
\hline 25 & 58.86 & 58.18 & 57.44 & 56.64 & 55.79 & 54.88 & 53.92 & 52.92 & 51.89 & 50.81 & 49.71 & 48.57 & 47.42 & 46.24 & 45.05 \\
\hline 26 & 59.42 & 58.75 & 58.02 & 57.22 & 56.37 & 55.46 & 54.51 & 53.51 & 52.47 & 51.39 & 50.28 & 49.14 & 47.98 & 46.80 & 45.59 \\
\hline 27 & 59.99 & 59.33 & 58.61 & 57.82 & 56.97 & 56.07 & 55.11 & 54.11 & 53.07 & 51.99 & 50.87 & 49.73 & 48.56 & 47.36 & 46.15 \\
\hline 28 & 60.59 & 59.94 & 59.22 & 58.43 & 57.59 & 56.69 & 55.73 & 54.73 & 53.69 & 52.60 & 51.48 & 50.33 & 49.15 & 47.95 & 46.73 \\
\hline 29 & 61.20 & 60.56 & 59.85 & 59.07 & 58.23 & 57.33 & 56.37 & 55.37 & 54.32 & 53.23 & 52.10 & 50.94 & 49.76 & 48.55 & 47.31 \\
\hline 30 & 61.84 & 61.21 & 60.50 & 59.73 & 58.89 & 57.99 & 57.04 & 56.03 & 54.98 & 53.88 & 52.75 & 51.58 & 50.38 & 49.16 & 47.92 \\
\hline 31 & 62.51 & 61.88 & 61.18 & 60.41 & 59.57 & 58.68 & 57.72 & 56.71 & 55.65 & 54.55 & 53.41 & 52.23 & 51.03 & 49.79 & 48.54 \\
\hline 32 & 63.20 & 62.58 & 61.89 & 61.12 & 60.28 & 59.39 & 58.43 & 57.41 & 56.35 & 55.24 & 54.09 & 52.91 & 51.69 & 50.44 & 49.17 \\
\hline
\end{tabular}




\section{Continued}

\begin{tabular}{|c|c|c|c|c|c|c|c|c|c|c|c|c|c|c|c|}
\hline 33 & 63.92 & 3.31 & 62.62 & 61.85 & 61.02 & 60.12 & 59.16 & 58.14 & 57.07 & 55.96 & 54.80 & 53.60 & 52.37 & 1.11 & 49.83 \\
\hline 34 & 64.67 & 4.06 & 63.38 & 6262 & 61.78 & 6088 & 59.92 & 8.89 & 57.82 & 56.69 & 55.52 & 4.31 & 53.07 & 1.80 & 0.50 \\
\hline 35 & 65.45 & .85 & 64.17 & 1 & 8 & 7 & 0 & 7 & 58.59 & 6 & 27 & 5 & & 51 & 1.19 \\
\hline 36 & 66.26 & .67 & 64.99 & 3 & 0 & 9 & 2 & 0.48 & 9 & 4 & 5 & 2 & 4 & 4 & 1.91 \\
\hline 37 & 67.12 & 66.53 & 65.85 & - 0.0 & 64.26 & 63.35 & 62.37 & 61.32 & 60.22 & 59.06 & 57.86 & 56.61 & 55.32 & .99 & 52.64 \\
\hline 38 & 68.01 & .42 & 66.75 & 99 & 5.15 & 4 & 5 & 2.20 & 61.08 & 59.91 & 58.69 & 7.42 & 6.12 & 4.77 & 3.40 \\
\hline 39 & 68.95 & 8.36 & 67.69 & 66.93 & 66.08 & 65.16 & 64.17 & 63.10 & 61.97 & 60.79 & 59.55 & 27 & 56.94 & .58 & 4.19 \\
\hline 40 & 69.93 & .34 & 68.67 & 67.91 & 67.06 & 3 & 2 & 5 & 0 & 61.70 & 60.45 & 4 & 0 & 41 & 5.00 \\
\hline 41 & 70.96 & 70.38 & 69.70 & 3.93 & 68.08 & 67.14 & 5.12 & 65.03 & 63.87 & 62.65 & 61.38 & .05 & 58 & 7.28 & 5.84 \\
\hline 42 & 72.04 & 71.46 & 70.78 & 70.00 & 69.14 & 68.19 & 67.16 & 66.06 & 64.88 & 63.64 & 62.35 & 61.00 & 59.60 & 8.17 & 56.70 \\
\hline 43 & 73.18 & .60 & 1 & 3 & 6 & 9 & 5 & 3 & 3 & 7 & 5 & 8 & 6 & 10 & .60 \\
\hline 44 & 74.39 & 73.80 & 73.11 & 2.31 & 71.43 & 45 & 39 & 68.25 & 3 & 74 & 40 & .00 & 55 & 0.06 & 8.53 \\
\hline 45 & 75.66 & 75.06 & 74.36 & 3.56 & 72.66 & 71.66 & 70.58 & 69.42 & 68.17 & 66.86 & 65.49 & 1.06 & 62.58 & 1.05 & 59.49 \\
\hline 46 & 77.01 & 76.40 & 75.69 & 87 & 73.95 & 72.94 & 83 & 70.64 & 69.37 & 68.03 & & & & 2.09 & 0.49 \\
\hline 47 & 78.43 & 7.81 & 77.08 & 625 & 75.31 & 74.27 & 14 & 71.93 & 70.63 & 69.26 & 82 & 32 & 64.76 & 3.16 & 1.52 \\
\hline 48 & 79.94 & 79.31 & 78.56 & 71 & 76.74 & 75 & 2 & 73.27 & 94 & 3 & 69.06 & & 2 & 4.28 & 2.60 \\
\hline 49 & 81.5 & .89 & 80.13 & 4 & 78.26 & 77.16 & 7 & 74.69 & 32 & 7 & 7 & 77 & 67 & 14 & 63.71 \\
\hline 50 & 83.25 & 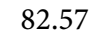 & 8 & 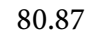 & 79.85 & & & 76.17 & 74 & 73 & 71 & .07 & 68 & & 4.87 \\
\hline 51 & 85.06 & 0 & 54 & 00 & 81.54 & 80.38 & 1 & 77.74 & 76 & 74.74 & 73.12 & 1.44 & 69 & 90 & 66.07 \\
\hline 52 & 87.00 & .26 & 40 & 43 & 33 & 12 & 80 & 9.39 & 88 & 76.28 & 1 & 87 & 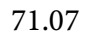 & 21 & 32 \\
\hline 53 & .06 & 8.29 & 39 & 7 & & & & 12 & 55 & 77.90 & & & & 70.57 & 8.61 \\
\hline 54 & 26 & 44 & 50 & 3 & 23 & & 18 & 95 & 32 & 60 & 79 & 92 & & 99 & 9.96 \\
\hline 55 & 1 & 75 & 9 & & 6 & & & 38 & & & & & & 47 & 1.35 \\
\hline 56 & 3 & & & & 91.62 & & & 2 & & & & 26 & 6 & 00 & 80 \\
\hline 57 & 8.83 & 97.84 & 96.70 & & & & & 7 & & & & 4 & 5 & 0 & 30 \\
\hline 58 & 3 & 5 & 99.44 & & & & & & & & & & & & \\
\hline 59 & 4.83 & 3.67 & 102.36 & 0.91 & (1) & & & 4 & & & & & & 79.97 & 77.47 \\
\hline 60 & 08.17 & 106.90 & 105.49 & 103.92 & 102.21 & 100.36 & 98.38 & 96.28 & & & & & & 81.75 & 79.13 \\
\hline 61 & 11.76 & 0.37 & 108.83 & 107.14 & 05.30 & 103.32 & 01.20 & 98.96 & 1 & 94.16 & & & & 60 & 80.83 \\
\hline 62 & 115.61 & 114.09 & 112.41 & 110.58 & 108.59 & 106.46 & 104.19 & 101.79 & 99.28 & 96.67 & 93.98 & 91.20 & 88.37 & 85.49 & 82.58 \\
\hline 63 & 119.76 & 118.09 & 116.25 & 114.25 & 112.10 & 109.79 & 107.35 & 104.78 & 102.09 & 99.31 & 96.44 & 93.49 & 90.49 & 87.44 & 84.37 \\
\hline 64 & 124.23 & 122.38 & 120.36 & 118.18 & 115.83 & 113.33 & 110.69 & 107.93 & 105.04 & 102.06 & 98.99 & 95.86 & 92.67 & 89.44 & 86.19 \\
\hline 65 & 29.05 & 127.00 & 124.77 & 122.37 & 119.81 & 117.09 & 114.23 & 111.24 & 108.13 & 104.93 & 101.64 & 98.29 & 94.90 & 91.47 & 88.02 \\
\hline
\end{tabular}


Table A3. Minimum daily efficiencies (in percent) for latitudes $\left[0^{\circ}, 65^{\circ}\right]$; slope angles $\left[31^{\circ}, 45^{\circ}\right]$.

\begin{tabular}{|c|c|c|c|c|c|c|c|c|c|c|c|c|c|c|c|}
\hline & 31 & 32 & 33 & 34 & 35 & 36 & 37 & 38 & 39 & 40 & 41 & 42 & 43 & 44 & 45 \\
\hline 0 & 41.73 & 41.61 & 41.53 & 41.50 & 41.51 & 41.56 & 41.66 & 41.80 & 41.98 & 42.21 & 42.48 & 42.80 & 43.16 & 43.56 & 44.02 \\
\hline 1 & 41.40 & 41.25 & 41.13 & 41.06 & 41.03 & 41.05 & 41.11 & 41.21 & 41.35 & 41.54 & 41.77 & 42.05 & 42.37 & 42.74 & 43.15 \\
\hline 2 & 41.11 & 40.92 & 40.77 & 40.66 & 40.59 & 40.57 & 40.59 & 40.66 & 40.76 & 40.91 & 41.11 & 41.34 & 41.62 & 41.95 & 42.32 \\
\hline 3 & 40.86 & 40.63 & 40.44 & 40.29 & 40.19 & 40.13 & 40.11 & 40.14 & 40.21 & 40.32 & 40.48 & 40.67 & 40.91 & 41.20 & 41.53 \\
\hline 4 & 40.64 & 40.37 & 40.14 & 39.96 & 39.82 & 39.72 & 39.67 & 39.66 & 39.69 & 39.76 & 39.88 & 40.04 & 40.24 & 40.49 & 40.78 \\
\hline 5 & 40.45 & 40.14 & 39.88 & 39.66 & 39.49 & 39.35 & 39.26 & 39.21 & 39.21 & 39.24 & 39.32 & 39.44 & 39.61 & 39.82 & 40.07 \\
\hline 6 & 40.30 & 39.96 & 39.65 & 39.40 & 39.18 & 39.01 & 38.89 & 38.80 & 38.76 & 38.76 & 38.80 & 38.88 & 39.01 & 39.18 & 39.39 \\
\hline 7 & 40.18 & 39.80 & 39.46 & 39.17 & 38.92 & 38.71 & 38.55 & 38.42 & 38.34 & 38.31 & 38.31 & 38.36 & 38.45 & 38.58 & 38.75 \\
\hline 8 & 40.10 & 39.68 & 39.30 & 38.97 & 38.68 & 38.44 & 38.24 & 38.08 & 37.96 & 37.89 & 37.86 & 37.87 & 37.92 & 38.01 & 38.15 \\
\hline 9 & 40.05 & 39.59 & 39.18 & 38.81 & 38.48 & 38.20 & 37.96 & 37.77 & 37.61 & 37.50 & 37.43 & 37.41 & 37.42 & 37.48 & 37.58 \\
\hline 10 & 40.03 & 39.54 & 39.08 & 38.68 & 38.31 & 38.00 & 37.72 & 37.49 & 37.30 & 37.15 & 37.05 & 36.98 & 36.96 & 36.98 & 37.04 \\
\hline 11 & 40.05 & 39.51 & 39.02 & 38.58 & 38.18 & 37.82 & 37.51 & 37.24 & 37.01 & 36.83 & 36.69 & 36.59 & 36.53 & 36.51 & 36.54 \\
\hline 12 & 40.10 & 39.53 & 39.00 & 38.51 & 38.08 & 37.68 & 37.33 & 37.03 & 36.76 & 36.54 & 36.36 & 36.23 & 36.13 & 36.08 & 36.07 \\
\hline 13 & 40.19 & 39.57 & 39.00 & 38.48 & 38.00 & 37.57 & 37.19 & 36.84 & 36.54 & 36.29 & 36.07 & 35.90 & 35.77 & 35.68 & 35.63 \\
\hline 14 & 40.30 & 39.65 & 39.04 & 38.48 & 37.96 & 37.49 & 37.07 & 36.69 & 36.35 & 36.06 & 35.81 & 35.60 & 35.43 & 35.31 & 35.22 \\
\hline 15 & 40.46 & 39.76 & 39.11 & 38.51 & 37.96 & 37.45 & 36.99 & 36.57 & 36.20 & 35.87 & 35.58 & 35.33 & 35.13 & 34.97 & 34.85 \\
\hline 16 & 40.64 & 39.90 & 39.21 & 38.57 & 37.98 & 37.43 & 36.93 & 36.48 & 36.07 & 35.70 & 35.38 & 35.09 & 34.86 & 34.66 & 34.50 \\
\hline 17 & 40.86 & 40.08 & 39.35 & 38.67 & 38.04 & 37.45 & 36.91 & 36.42 & 35.97 & 35.57 & 35.21 & 34.89 & 34.61 & 34.38 & 34.19 \\
\hline 18 & 41.11 & 40.29 & 39.52 & 38.79 & 38.12 & 37.50 & 36.92 & 36.39 & 35.90 & 35.46 & 35.06 & 34.71 & 34.40 & 34.13 & 33.90 \\
\hline 19 & 41.40 & 40.53 & 39.72 & 38.95 & 38.24 & 37.58 & 36.96 & 36.39 & 35.86 & 35.39 & 34.95 & 34.56 & 34.21 & 33.91 & 33.64 \\
\hline 20 & 41.72 & 40.81 & 39.95 & 39.14 & 38.39 & 37.68 & 37.03 & 36.42 & 35.86 & 35.34 & 34.87 & 34.44 & 34.06 & 33.71 & 33.42 \\
\hline 21 & 42.08 & 41.12 & 40.22 & 39.37 & 38.57 & 37.82 & 37.13 & 36.48 & 35.88 & 35.32 & 34.81 & 34.35 & 33.93 & 33.55 & 33.22 \\
\hline 22 & 42.47 & 41.46 & 40.52 & 39.62 & 38.78 & 37.99 & 37.26 & 36.57 & 35.93 & 35.33 & 34.79 & 34.29 & 33.83 & 33.41 & 33.04 \\
\hline 23 & 42.89 & 41.84 & 40.85 & 39.91 & 39.03 & 38.20 & 37.42 & 36.69 & 36.01 & 35.38 & 34.79 & 34.25 & 33.76 & 33.30 & 32.90 \\
\hline 24 & 43.35 & 42.25 & 41.21 & 40.23 & 39.30 & 38.43 & 37.61 & 36.84 & 36.12 & 35.44 & 34.82 & 34.24 & 33.71 & 33.22 & 32.78 \\
\hline 25 & 43.85 & 42.70 & 41.61 & 40.58 & 39.61 & 38.69 & 37.82 & 37.01 & 36.25 & 35.54 & 34.88 & 34.26 & 33.69 & 33.17 & 32.69 \\
\hline 26 & 44.38 & 43.18 & 42.04 & 40.96 & 39.94 & 38.98 & 38.07 & 37.22 & 36.42 & 35.67 & 34.96 & 34.31 & 33.70 & 33.14 & 32.62 \\
\hline 27 & 44.93 & 43.69 & 42.50 & 41.38 & 40.31 & 39.30 & 38.35 & 37.46 & 36.61 & 35.82 & 35.08 & 34.38 & 33.74 & 33.14 & 32.58 \\
\hline 28 & 45.49 & 44.24 & 43.00 & 41.83 & 40.71 & 39.66 & 38.66 & 37.72 & 36.83 & 36.00 & 35.22 & 34.48 & 33.80 & 33.16 & 32.57 \\
\hline 29 & 46.06 & 44.80 & 43.54 & 42.31 & 41.15 & 40.04 & 39.00 & 38.01 & 37.08 & 36.21 & 35.38 & 34.61 & 33.88 & 33.21 & 32.58 \\
\hline 30 & 46.66 & 45.38 & 44.10 & 42.82 & 41.61 & 40.46 & 39.37 & 38.34 & 37.36 & 36.44 & 35.58 & 34.76 & 34.00 & 33.28 & 32.61 \\
\hline 31 & 47.26 & 45.97 & 44.67 & 43.37 & 42.10 & 40.90 & 39.77 & 38.69 & 37.67 & 36.70 & 35.79 & 34.94 & 34.13 & 33.38 & 32.67 \\
\hline 32 & 47.88 & 46.58 & 45.27 & 43.94 & 42.63 & 41.38 & 40.19 & 39.07 & 38.00 & 36.99 & 36.04 & 35.14 & 34.29 & 33.50 & 32.75 \\
\hline
\end{tabular}




\section{Continued}

\begin{tabular}{|c|c|c|c|c|c|c|c|c|c|c|c|c|c|c|c|}
\hline 33 & 48.52 & 47.20 & 45.87 & 44.53 & 43.19 & 41.89 & 40.65 & 39.47 & 38.36 & 37.31 & 36.31 & 35.37 & 34.48 & 33.65 & 32.86 \\
\hline 34 & 49.18 & 47.84 & 46.50 & 45.14 & 43.78 & 42.42 & 41.13 & 39.91 & 38.75 & 37.65 & 36.61 & 35.62 & 34.69 & 33.82 & 32.99 \\
\hline 35 & 49.86 & 48.50 & 47.14 & 45.76 & 44.38 & 42.99 & 41.65 & 40.37 & 39.16 & 38.02 & 36.93 & 35.90 & 34.93 & 34.01 & 33.14 \\
\hline 36 & 50.55 & 49.18 & 47.79 & 46.40 & 44.99 & 43.59 & 42.19 & 40.87 & 39.61 & 38.41 & 37.28 & 36.20 & 35.18 & 34.22 & 33.31 \\
\hline 37 & 51.27 & 49.88 & 48.47 & 47.05 & 45.62 & 44.19 & 42.77 & 41.39 & 40.07 & 38.83 & 37.65 & 36.53 & 35.46 & 34.46 & 33.51 \\
\hline 38 & 52.01 & 50.59 & 49.16 & 47.72 & 46.27 & 44.82 & 43.37 & 41.93 & 40.57 & 39.27 & 38.04 & 36.87 & 35.77 & 34.72 & 33.72 \\
\hline 39 & 52.77 & 51.33 & 49.88 & 48.41 & 46.94 & 45.46 & 43.98 & 42.51 & 41.09 & 39.74 & 38.46 & 37.24 & 36.09 & 35.00 & 33.96 \\
\hline 40 & 53.56 & 52.09 & 50.61 & 49.12 & 47.62 & 46.11 & 44.61 & 43.11 & 41.63 & 40.23 & 38.90 & 37.64 & 36.43 & 35.30 & 34.22 \\
\hline 41 & 54.37 & 52.88 & 51.37 & 49.85 & 48.32 & 46.79 & 45.25 & 43.72 & 42.20 & 40.75 & 39.36 & 38.05 & 36.80 & 35.61 & 34.49 \\
\hline 42 & 55.21 & 53.69 & 52.15 & 50.60 & 49.04 & 47.48 & 45.91 & 44.35 & 42.80 & 41.29 & 39.85 & 38.48 & 37.18 & 35.95 & 34.78 \\
\hline 43 & 56.07 & 54.52 & 52.95 & 51.37 & 49.78 & 48.18 & 46.59 & 45.00 & 43.41 & 41.85 & 40.35 & 38.94 & 37.59 & 36.31 & 35.09 \\
\hline 44 & 56.97 & 55.39 & 53.78 & 52.17 & 50.54 & 48.91 & 47.28 & 45.65 & 44.03 & 42.43 & 40.88 & 39.41 & 38.01 & 36.68 & 35.41 \\
\hline 45 & 57.90 & 56.28 & 54.64 & 52.99 & 51.32 & 49.66 & 47.99 & 46.33 & 44.67 & 43.03 & 41.42 & 39.90 & 38.44 & 37.06 & 35.75 \\
\hline 46 & 58.86 & 57.20 & 55.52 & 53.83 & 52.13 & 50.42 & 48.72 & 47.02 & 45.32 & 43.64 & 41.99 & 40.40 & 38.90 & 37.46 & 36.10 \\
\hline 47 & 59.85 & 58.15 & 56.43 & 54.70 & 52.96 & 51.21 & 49.46 & 47.72 & 45.99 & 44.27 & 42.56 & 40.92 & 39.36 & 37.88 & 36.47 \\
\hline 48 & 60.88 & 59.14 & 57.37 & 55.59 & 53.81 & 52.01 & 50.22 & 48.44 & 46.66 & 44.90 & 43.16 & 41.46 & 39.84 & 38.30 & 36.84 \\
\hline 49 & 61.95 & 60.15 & 58.34 & 56.52 & 54.68 & 52.84 & 51.00 & 49.17 & 47.35 & 45.55 & 43.76 & 42.00 & 40.33 & 38.74 & 37.22 \\
\hline 50 & 63.05 & 61.21 & 59.34 & 57.47 & 55.58 & 53.69 & 51.80 & 49.92 & 48.05 & 46.20 & 44.37 & 42.56 & 40.83 & 39.18 & 37.61 \\
\hline 51 & 64.20 & 62.30 & 60.38 & 58.44 & 56.50 & 54.56 & 52.62 & 50.68 & 48.76 & 46.86 & 44.98 & 43.12 & 41.33 & 39.62 & 38.00 \\
\hline 52 & 65.38 & 63.42 & 61.44 & 59.45 & 57.45 & 55.45 & 53.45 & 51.46 & 49.49 & 47.53 & 45.60 & 43.69 & 41.84 & 40.07 & 38.39 \\
\hline 53 & 66.61 & 64.59 & 62.54 & 60.48 & 58.42 & 56.35 & 54.30 & 52.25 & 50.22 & 48.20 & 46.22 & 44.25 & 42.34 & 40.52 & 38.79 \\
\hline 54 & 67.89 & 65.79 & 63.67 & 61.55 & 59.41 & 57.28 & 55.16 & 53.05 & 50.95 & 48.88 & 46.84 & 44.82 & 42.85 & 40.97 & 39.17 \\
\hline 55 & 69.21 & 67.03 & 64.84 & 62.64 & 60.43 & 58.23 & 56.03 & 53.85 & 51.69 & 49.56 & 47.45 & 45.38 & 43.34 & 41.40 & 39.55 \\
\hline 56 & 70.57 & 68.31 & 66.04 & 63.75 & 61.47 & 59.19 & 56.92 & 54.67 & 52.44 & 50.23 & 48.06 & 45.93 & 43.83 & 41.83 & 39.92 \\
\hline 57 & 71.98 & 69.63 & 67.26 & 64.89 & 62.52 & 60.16 & 57.81 & 55.48 & 53.18 & 50.90 & 48.66 & 46.46 & 44.30 & 42.24 & 40.27 \\
\hline 58 & 73.43 & 70.98 & 68.52 & 66.05 & 63.59 & 61.14 & 58.70 & 56.29 & 53.91 & 51.56 & 49.25 & 46.98 & 44.75 & 42.62 & 40.60 \\
\hline 59 & 74.93 & 72.37 & 69.80 & 67.24 & 64.67 & 62.13 & 59.60 & 57.10 & 54.63 & 52.20 & 49.82 & 47.47 & 45.18 & 42.98 & 40.90 \\
\hline 60 & 76.47 & 73.79 & 71.11 & 68.43 & 65.76 & 63.11 & 60.49 & 57.89 & 55.34 & 52.82 & 50.35 & 47.93 & 45.57 & 43.31 & 41.16 \\
\hline 61 & 78.04 & 75.24 & 72.43 & 69.64 & 66.85 & 64.09 & 61.36 & 58.67 & 56.02 & 53.41 & 50.86 & 48.35 & 45.92 & 43.59 & 41.39 \\
\hline 62 & 79.65 & 76.71 & 73.77 & 70.84 & 67.93 & 65.05 & 62.21 & 59.41 & 56.66 & 53.96 & 51.31 & 48.72 & 46.21 & 43.83 & 41.56 \\
\hline 63 & 81.28 & 78.19 & 75.10 & 72.03 & 68.99 & 65.99 & 63.03 & 60.11 & 57.25 & 54.45 & 51.71 & 49.03 & 46.45 & 44.00 & 41.67 \\
\hline 64 & 82.93 & 79.67 & 76.42 & 73.20 & 70.02 & 66.88 & 63.79 & 60.76 & 57.78 & 54.88 & 52.04 & 49.27 & 46.62 & 44.10 & 41.72 \\
\hline 65 & 84.57 & 81.13 & 77.72 & 74.34 & 71.00 & 67.72 & 64.49 & 61.33 & 58.24 & 55.22 & 52.28 & 49.42 & 46.70 & 44.12 & 41.68 \\
\hline
\end{tabular}


Table A4. Minimum daily efficiencies (in percent) for latitudes $\left[0^{\circ}, 65^{\circ}\right]$; slope angles $\left[46^{\circ}, 60^{\circ}\right]$.

\begin{tabular}{|c|c|c|c|c|c|c|c|c|c|c|c|c|c|c|c|}
\hline & 46 & 47 & 48 & 49 & 50 & 51 & 52 & 53 & 54 & 55 & 56 & 57 & 58 & 59 & 60 \\
\hline 0 & 44.52 & 45.06 & 45.66 & 46.31 & 47.00 & 47.75 & 48.55 & 49.41 & 50.32 & 51.29 & 52.32 & 53.41 & 54.57 & 55.79 & 57.07 \\
\hline 1 & 43.60 & 44.11 & 44.66 & 45.26 & 45.91 & 46.61 & 47.37 & 48.17 & 49.03 & 49.95 & 50.92 & 51.96 & 53.05 & 54.21 & 55.43 \\
\hline 2 & 42.73 & 43.20 & 43.70 & 44.26 & 44.87 & 45.52 & 46.23 & 46.98 & 47.79 & 48.66 & 49.58 & 50.56 & 51.60 & 52.70 & 53.86 \\
\hline 3 & 41.90 & 42.32 & 42.79 & 43.30 & 43.86 & 44.47 & 45.13 & 45.84 & 46.60 & 47.42 & 48.29 & 49.21 & 50.19 & 51.24 & 52.34 \\
\hline 4 & 41.11 & 41.49 & 41.92 & 42.39 & 42.90 & 43.47 & 44.08 & 44.75 & 45.46 & 46.23 & 47.04 & 47.92 & 48.85 & 49.83 & 50.88 \\
\hline 5 & 40.36 & 40.70 & 41.08 & 41.51 & 41.99 & 42.51 & 43.08 & 43.69 & 44.36 & 45.08 & 45.85 & 46.67 & 47.55 & 48.48 & 49.47 \\
\hline 6 & 39.65 & 39.95 & 40.29 & 40.68 & 41.11 & 41.59 & 42.11 & 42.69 & 43.31 & 43.98 & 44.70 & 45.47 & 46.30 & 47.18 & 48.12 \\
\hline 7 & 38.97 & 39.23 & 39.53 & 39.88 & 40.27 & 40.71 & 41.19 & 41.72 & 42.30 & 42.92 & 43.60 & 44.32 & 45.10 & 45.93 & 46.82 \\
\hline 8 & 38.33 & 38.55 & 38.81 & 39.12 & 39.47 & 39.87 & 40.31 & 40.79 & 41.33 & 41.91 & 42.54 & 43.22 & 43.95 & 44.73 & 45.56 \\
\hline 9 & 37.72 & 37.90 & 38.13 & 38.39 & 38.71 & 39.06 & 39.46 & 39.91 & 40.40 & 40.94 & 41.52 & 42.16 & 42.84 & 43.57 & 44.36 \\
\hline 10 & 37.14 & 37.29 & 37.48 & 37.71 & 37.98 & 38.30 & 38.66 & 39.06 & 39.51 & 40.01 & 40.55 & 41.14 & 41.77 & 42.46 & 43.20 \\
\hline 11 & 36.60 & 36.71 & 36.86 & 37.05 & 37.29 & 37.57 & 37.89 & 38.25 & 38.66 & 39.11 & 39.61 & 40.16 & 40.75 & 41.39 & 42.08 \\
\hline 12 & 36.10 & 36.17 & 36.28 & 36.44 & 36.63 & 36.87 & 37.15 & 37.48 & 37.85 & 38.26 & 38.72 & 39.22 & 39.77 & 40.37 & 41.01 \\
\hline 13 & 35.62 & 35.66 & 35.73 & 35.85 & 36.01 & 36.21 & 36.45 & 36.74 & 37.07 & 37.44 & 37.86 & 38.32 & 38.83 & 39.38 & 39.98 \\
\hline 14 & 35.18 & 35.18 & 35.22 & 35.30 & 35.42 & 35.58 & 35.79 & 36.04 & 36.33 & 36.66 & 37.04 & 37.46 & 37.92 & 38.43 & 38.99 \\
\hline 15 & 34.77 & 34.73 & 34.73 & 34.78 & 34.86 & 34.99 & 35.16 & 35.37 & 35.62 & 35.91 & 36.25 & 36.63 & 37.06 & 37.53 & 38.04 \\
\hline 16 & 34.39 & 34.31 & 34.28 & 34.29 & 34.34 & 34.43 & 34.56 & 34.73 & 34.95 & 35.20 & 35.50 & 35.84 & 36.23 & 36.65 & 37.13 \\
\hline 17 & 34.04 & 33.93 & 33.86 & 33.83 & 33.84 & 33.90 & 33.99 & 34.13 & 34.31 & 34.52 & 34.78 & 35.09 & 35.43 & 35.82 & 36.25 \\
\hline 18 & 33.71 & 33.57 & 33.47 & 33.40 & 33.38 & 33.40 & 33.46 & 33.56 & 33.70 & 33.88 & 34.10 & 34.36 & 34.67 & 35.02 & 35.41 \\
\hline 19 & 33.42 & 33.24 & 33.10 & 33.00 & 32.94 & 32.93 & 32.95 & 33.01 & 33.12 & 33.26 & 33.45 & 33.68 & 33.94 & 34.25 & 34.61 \\
\hline 20 & 33.16 & 32.94 & 32.77 & 32.63 & 32.54 & 32.49 & 32.47 & 32.50 & 32.57 & 32.68 & 32.83 & 33.02 & 33.25 & 33.52 & 33.84 \\
\hline 21 & 32.92 & 32.67 & 32.46 & 32.29 & 32.16 & 32.07 & 32.03 & 32.02 & 32.05 & 32.12 & 32.24 & 32.39 & 32.58 & 32.82 & 33.10 \\
\hline 22 & 32.71 & 32.43 & 32.18 & 31.98 & 31.81 & 31.69 & 31.61 & 31.56 & 31.56 & 31.60 & 31.68 & 31.79 & 31.95 & 32.15 & 32.39 \\
\hline 23 & 32.53 & 32.21 & 31.93 & 31.69 & 31.49 & 31.33 & 31.21 & 31.14 & 31.10 & 31.10 & 31.14 & 31.23 & 31.35 & 31.51 & 31.71 \\
\hline 24 & 32.38 & 32.02 & 31.70 & 31.43 & 31.20 & 31.00 & 30.85 & 30.74 & 30.66 & 30.63 & 30.64 & 30.69 & 30.77 & 30.90 & 31.07 \\
\hline 25 & 32.25 & 31.86 & 31.50 & 31.20 & 30.93 & 30.70 & 30.51 & 30.36 & 30.26 & 30.19 & 30.16 & 30.17 & 30.23 & 30.32 & 30.45 \\
\hline 26 & 32.15 & 31.72 & 31.33 & 30.99 & 30.68 & 30.42 & 30.20 & 30.02 & 29.88 & 29.77 & 29.71 & 29.69 & 29.71 & 29.76 & 29.86 \\
\hline 27 & 32.07 & 31.61 & 31.18 & 30.80 & 30.46 & 30.17 & 29.91 & 29.69 & 29.52 & 29.38 & 29.29 & 29.23 & 29.21 & 29.23 & 29.30 \\
\hline 28 & 32.02 & 31.52 & 31.06 & 30.64 & 30.27 & 29.94 & 29.65 & 29.40 & 29.19 & 29.02 & 28.89 & 28.80 & 28.74 & 28.73 & 28.76 \\
\hline 29 & 31.99 & 31.45 & 30.96 & 30.51 & 30.10 & 29.73 & 29.41 & 29.12 & 28.88 & 28.68 & 28.51 & 28.39 & 28.30 & 28.25 & 28.25 \\
\hline 30 & 31.99 & 31.42 & 30.88 & 30.40 & 29.95 & 29.55 & 29.19 & 28.87 & 28.59 & 28.36 & 28.16 & 28.00 & 27.88 & 27.80 & 27.76 \\
\hline 31 & 32.01 & 31.40 & 30.83 & 30.31 & 29.83 & 29.39 & 29.00 & 28.65 & 28.33 & 28.06 & 27.83 & 27.64 & 27.48 & 27.37 & 27.30 \\
\hline 32 & 32.06 & 31.41 & 30.80 & 30.24 & 29.73 & 29.26 & 28.83 & 28.44 & 28.09 & 27.79 & 27.52 & 27.30 & 27.11 & 26.96 & 26.86 \\
\hline
\end{tabular}




\section{Continued}

\begin{tabular}{|c|c|c|c|c|c|c|c|c|c|c|c|c|c|c|c|}
\hline 33 & 32.12 & 31.44 & 30.79 & 30.20 & 29.65 & 29.14 & 28.68 & 28.25 & 27.87 & 27.53 & 27.24 & 26.98 & 26.76 & 26.58 & 26.44 \\
\hline 34 & 32.21 & 31.49 & 30.81 & 30.18 & 29.59 & 29.05 & 28.55 & 28.09 & 27.68 & 27.30 & 26.97 & 26.68 & 26.43 & 26.21 & 26.04 \\
\hline 35 & 32.33 & 31.56 & 30.84 & 30.17 & 29.55 & 28.97 & 28.44 & 27.95 & 27.50 & 27.09 & 26.72 & 26.40 & 26.11 & 25.87 & 25.66 \\
\hline 36 & 32.46 & 31.65 & 30.90 & 30.19 & 29.53 & 28.92 & 28.35 & 27.82 & 27.34 & 26.90 & 26.50 & 26.14 & 25.82 & 25.54 & 25.30 \\
\hline 37 & 32.61 & 31.77 & 30.97 & 30.23 & 29.53 & 28.88 & 28.28 & 27.71 & 27.20 & 26.72 & 26.29 & 25.90 & 25.54 & 25.23 & 24.96 \\
\hline 38 & 32.79 & 31.90 & 31.07 & 30.29 & 29.55 & 28.86 & 28.22 & 27.62 & 27.07 & 26.56 & 26.10 & 25.67 & 25.29 & 24.94 & 24.64 \\
\hline 39 & 32.98 & 32.05 & 31.18 & 30.36 & 29.59 & 28.86 & 28.18 & 27.55 & 26.96 & 26.42 & 25.92 & 25.46 & 25.04 & 24.67 & 24.33 \\
\hline 40 & 33.19 & 32.23 & 31.31 & 30.45 & 29.64 & 28.88 & 28.16 & 27.50 & 26.87 & 26.29 & 25.76 & 25.27 & 24.82 & 24.41 & 24.04 \\
\hline 41 & 33.42 & 32.41 & 31.46 & 30.56 & 29.71 & 28.91 & 28.16 & 27.45 & 26.80 & 26.18 & 25.61 & 25.09 & 24.60 & 24.16 & 23.76 \\
\hline 42 & 33.67 & 32.62 & 31.62 & 30.68 & 29.79 & 28.95 & 28.17 & 27.42 & 26.73 & 26.08 & 25.48 & 24.92 & 24.40 & 23.93 & 23.50 \\
\hline 43 & 33.93 & 32.84 & 31.80 & 30.82 & 29.89 & 29.01 & 28.19 & 27.41 & 26.68 & 26.00 & 25.36 & 24.77 & 24.22 & 23.71 & 23.24 \\
\hline 44 & 34.21 & 33.07 & 31.99 & 30.97 & 30.00 & 29.08 & 28.22 & 27.41 & 26.64 & 25.92 & 25.25 & 24.62 & 24.04 & 23.50 & 23.00 \\
\hline 45 & 34.50 & 33.32 & 32.19 & 31.13 & 30.12 & 29.16 & 28.26 & 27.41 & 26.61 & 25.86 & 25.15 & 24.49 & 23.87 & 23.30 & 22.77 \\
\hline 46 & 34.81 & 33.58 & 32.41 & 31.30 & 30.25 & 29.26 & 28.31 & 27.43 & 26.59 & 25.80 & 25.06 & 24.36 & 23.71 & 23.11 & 22.54 \\
\hline 47 & 35.12 & 33.85 & 32.63 & 31.48 & 30.39 & 29.35 & 28.37 & 27.45 & 26.57 & 25.75 & 24.97 & 24.24 & 23.56 & 22.92 & 22.32 \\
\hline 48 & 35.45 & 34.12 & 32.86 & 31.67 & 30.54 & 29.46 & 28.44 & 27.48 & 26.56 & 25.70 & 24.89 & 24.13 & 23.41 & 22.74 & 22.11 \\
\hline 49 & 35.78 & 34.41 & 33.10 & 31.86 & 30.69 & 29.57 & 28.51 & 27.51 & 26.56 & 25.66 & 24.81 & 24.01 & 23.26 & 22.56 & 21.90 \\
\hline 50 & 36.12 & 34.70 & 33.34 & 32.06 & 30.84 & 29.68 & 28.58 & 27.54 & 26.55 & 25.62 & 24.74 & 23.90 & 23.12 & 22.38 & 21.69 \\
\hline 51 & 36.46 & 34.99 & 33.59 & 32.26 & 31.00 & 29.79 & 28.65 & 27.57 & 26.55 & 25.58 & 24.66 & 23.79 & 22.97 & 22.20 & 21.47 \\
\hline 52 & 36.80 & 35.28 & 33.83 & 32.46 & 31.15 & 29.90 & 28.72 & 27.60 & 26.54 & 25.53 & 24.58 & 23.67 & 22.82 & 22.02 & 21.26 \\
\hline 53 & 37.14 & 35.57 & 34.07 & 32.65 & 31.30 & 30.01 & 28.79 & 27.63 & 26.52 & 25.48 & 24.49 & 23.55 & 22.66 & 21.83 & 21.04 \\
\hline 54 & 37.47 & 35.85 & 34.30 & 32.83 & 31.44 & 30.11 & 28.84 & 27.64 & 26.50 & 25.42 & 24.39 & 23.42 & 22.50 & 21.63 & 20.81 \\
\hline 55 & 37.79 & 36.12 & 34.53 & 33.01 & 1.57 & 30.19 & 28.89 & 27.65 & 26.47 & 25.35 & 24.28 & 23.27 & 22.32 & 21.42 & 20.56 \\
\hline 56 & 38.11 & 36.38 & 34.73 & 33.17 & 31.68 & 30.26 & 28.92 & 27.63 & 26.42 & 25.26 & 24.16 & 23.12 & 22.13 & 21.19 & 20.31 \\
\hline 57 & 38.40 & 36.62 & 34.92 & 33.31 & 31.78 & 30.32 & 28.93 & 27.60 & 26.35 & 25.15 & 24.02 & 22.94 & 21.92 & 20.95 & 20.03 \\
\hline 58 & 38.67 & 36.84 & 35.09 & 33.43 & 31.85 & 30.34 & 28.91 & 27.55 & 26.25 & 25.02 & 23.85 & 22.74 & 21.68 & 20.68 & 19.73 \\
\hline 59 & 38.91 & 37.03 & 35.23 & 33.52 & 31.89 & 30.34 & 28.87 & 27.46 & 26.13 & 24.86 & 23.65 & 22.51 & 21.42 & 20.38 & 19.40 \\
\hline 60 & 39.12 & 37.18 & 35.33 & 33.57 & 31.90 & 30.31 & 28.79 & 27.35 & 25.97 & 24.67 & 23.42 & 22.24 & 21.12 & 20.06 & 19.05 \\
\hline 61 & 39.29 & 37.29 & 35.39 & 33.59 & 31.87 & 30.23 & 28.67 & 27.19 & 25.78 & 24.43 & 23.16 & 21.94 & 20.79 & 19.69 & 18.65 \\
\hline 62 & 39.40 & 37.35 & 35.40 & 33.55 & 31.78 & 30.10 & 28.50 & 26.98 & 25.53 & 24.15 & 22.84 & 21.60 & 20.41 & 19.28 & 18.21 \\
\hline 63 & 39.46 & 37.36 & 35.36 & 33.45 & 31.64 & 29.92 & 28.28 & 26.72 & 25.24 & 23.82 & 22.48 & 21.20 & 19.98 & 18.82 & 17.72 \\
\hline 64 & 39.45 & 37.29 & 35.24 & 33.29 & 31.44 & 29.67 & 28.00 & 26.40 & 24.88 & 23.43 & 22.05 & 20.74 & 19.49 & 18.30 & 17.18 \\
\hline 65 & 39.35 & 37.15 & 35.05 & 33.05 & 31.16 & 29.35 & 27.63 & 26.00 & 24.44 & 22.96 & 21.55 & 20.21 & 18.94 & 17.72 & 16.57 \\
\hline
\end{tabular}


Table A5. Minimum daily efficiencies (in percent) for latitudes $\left[0^{\circ}, 65^{\circ}\right]$; slope angles $\left[61^{\circ}, 75^{\circ}\right]$.

\begin{tabular}{|c|c|c|c|c|c|c|c|c|c|c|c|c|c|c|c|}
\hline & 61 & 62 & 63 & 64 & 65 & 66 & 67 & 68 & 69 & 70 & 71 & 72 & 73 & 74 & 75 \\
\hline 0 & 58.43 & 59.86 & 61.36 & 62.95 & 64.61 & 66.36 & 68.20 & 70.14 & 72.17 & 74.31 & 76.55 & 78.91 & 81.38 & 83.99 & 86.73 \\
\hline 1 & 56.73 & 58.09 & 59.52 & 61.03 & 62.62 & 64.29 & 66.05 & 67.89 & 69.83 & 71.87 & 74.02 & 76.27 & 78.63 & 81.11 & 83.73 \\
\hline 2 & 55.09 & 56.38 & 57.75 & 59.19 & 60.70 & 62.30 & 63.97 & 65.73 & 67.59 & 69.53 & 71.58 & 73.73 & 75.98 & 78.35 & 80.85 \\
\hline 3 & 53.51 & 54.74 & 56.04 & 57.41 & 58.85 & 60.37 & 61.97 & 63.65 & 65.42 & 67.28 & 69.23 & 71.28 & 73.44 & 75.70 & 78.08 \\
\hline 4 & 51.99 & 53.16 & 54.39 & 55.70 & 57.07 & 58.52 & 60.05 & 61.65 & 63.34 & 65.11 & 66.97 & 68.93 & 70.99 & 73.15 & 75.42 \\
\hline 5 & 50.52 & 51.63 & 52.81 & 54.05 & 55.36 & 56.74 & 58.19 & 59.72 & 61.33 & 63.02 & 64.80 & 66.67 & 68.63 & 70.69 & 72.86 \\
\hline 6 & 49.11 & 50.17 & 51.28 & 52.46 & 53.71 & 55.02 & 56.41 & 57.86 & 59.40 & 61.01 & 62.71 & 64.49 & 66.36 & 68.33 & 70.40 \\
\hline 7 & 47.76 & 48.76 & 49.81 & 50.93 & 52.12 & 53.37 & 54.68 & 56.07 & 57.53 & 59.07 & 60.69 & 62.39 & 64.18 & 66.06 & 68.03 \\
\hline 8 & 46.45 & 47.40 & 48.40 & 49.46 & 50.59 & 51.77 & 53.03 & 54.35 & 55.74 & 57.21 & 58.75 & 60.37 & 62.08 & 63.87 & 65.76 \\
\hline 9 & 45.20 & 46.09 & 47.04 & 48.04 & 49.11 & 50.24 & 51.43 & 52.69 & 54.01 & 55.41 & 56.88 & 58.43 & 60.06 & 61.77 & 63.56 \\
\hline 10 & 43.99 & 44.83 & 45.72 & 46.68 & 47.69 & 48.76 & 49.89 & 51.09 & 52.35 & 53.68 & 55.08 & 56.55 & 58.11 & 59.74 & 61.45 \\
\hline 11 & 42.82 & 43.62 & 44.46 & 45.36 & 46.32 & 47.33 & 48.41 & 49.54 & 50.74 & 52.01 & 53.34 & 54.75 & 56.23 & 57.78 & 59.42 \\
\hline 12 & 41.70 & 42.45 & 43.25 & 44.10 & 45.00 & 45.96 & 46.98 & 48.06 & 49.20 & 50.40 & 51.67 & 53.01 & 54.42 & 55.90 & 57.46 \\
\hline 13 & 40.63 & 41.33 & 42.08 & 42.88 & 43.73 & 44.64 & 45.60 & 46.62 & 47.70 & 48.85 & 50.06 & 51.33 & 52.67 & 54.08 & 55.57 \\
\hline 14 & 39.59 & 40.25 & 40.95 & 41.70 & 42.51 & 43.36 & 44.27 & 45.24 & 46.27 & 47.35 & 48.50 & 49.71 & 50.99 & 52.33 & 53.75 \\
\hline 15 & 38.60 & 39.21 & 39.87 & 40.57 & 41.33 & 42.13 & 42.99 & 43.91 & 44.88 & 45.91 & 47.00 & 48.15 & 49.37 & 50.65 & 52.00 \\
\hline 16 & 37.65 & 38.21 & 38.82 & 39.48 & 40.19 & 40.95 & 41.76 & 42.63 & 43.55 & 44.52 & 45.55 & 46.65 & 47.80 & 49.02 & 50.31 \\
\hline 17 & 36.73 & 37.25 & 37.82 & 38.44 & 39.10 & 39.81 & 40.57 & 41.39 & 42.26 & 43.18 & 44.16 & 45.20 & 46.29 & 47.45 & 48.67 \\
\hline 18 & 35.85 & 36.33 & 36.86 & 37.43 & 38.05 & 38.71 & 39.43 & 40.20 & 41.02 & 41.89 & 42.81 & 43.80 & 44.84 & 45.94 & 47.10 \\
\hline 19 & 35.00 & 35.44 & 35.93 & 36.46 & 37.03 & 37.66 & 38.33 & 39.05 & 39.82 & 40.64 & 41.52 & 42.45 & 43.43 & 44.47 & 45.58 \\
\hline 20 & 34.19 & 34.59 & 35.04 & 35.53 & 36.06 & 36.64 & 37.27 & 37.94 & 38.66 & 39.44 & 40.26 & 41.14 & 42.08 & 43.06 & 44.11 \\
\hline 21 & 33.42 & 33.78 & 34.18 & 34.63 & 35.12 & 35.66 & 36.24 & 36.87 & 37.55 & 38.28 & 39.06 & 39.88 & 40.77 & 41.70 & 42.70 \\
\hline 22 & 32.67 & 32.99 & 33.36 & 33.77 & 34.22 & 34.72 & 35.26 & 35.84 & 36.48 & 37.16 & 37.89 & 38.67 & 39.50 & 40.39 & 41.33 \\
\hline 23 & 31.96 & 32.24 & 32.57 & 32.94 & 33.35 & 33.81 & 34.31 & 34.85 & 35.44 & 36.08 & 36.76 & 37.50 & 38.28 & 39.12 & 40.01 \\
\hline 24 & 31.27 & 31.52 & 31.81 & 32.14 & 32.52 & 32.93 & 33.39 & 33.90 & 34.44 & 35.04 & 35.68 & 36.37 & 37.11 & 37.89 & 38.73 \\
\hline 25 & 30.62 & 30.83 & 31.08 & 31.38 & 31.71 & 32.09 & 32.51 & 32.97 & 33.48 & 34.03 & 34.63 & 35.27 & 35.97 & 36.71 & 37.50 \\
\hline 26 & 29.99 & 30.17 & 30.39 & 30.64 & 30.94 & 31.28 & 31.66 & 32.08 & 32.55 & 33.06 & 33.62 & 34.22 & 34.87 & 35.56 & 36.31 \\
\hline 27 & 29.40 & 29.54 & 29.72 & 29.94 & 30.20 & 30.50 & 30.84 & 31.23 & 31.66 & 32.13 & 32.64 & 33.20 & 33.81 & 34.46 & 35.16 \\
\hline 28 & 28.82 & 28.93 & 29.08 & 29.26 & 29.48 & 29.75 & 30.06 & 30.40 & 30.79 & 31.22 & 31.70 & 32.22 & 32.78 & 33.39 & 34.04 \\
\hline 29 & 28.28 & 28.35 & 28.46 & 28.61 & 28.80 & 29.03 & 29.30 & 29.61 & 29.96 & 30.35 & 30.79 & 31.26 & 31.79 & 32.35 & 32.97 \\
\hline 30 & 27.76 & 27.79 & 27.87 & 27.98 & 28.14 & 28.33 & 28.57 & 28.84 & 29.15 & 29.51 & 29.91 & 30.34 & 30.83 & 31.35 & 31.92 \\
\hline 31 & 27.26 & 27.26 & 27.30 & 27.39 & 27.50 & 27.66 & 27.86 & 28.10 & 28.38 & 28.70 & 29.05 & 29.46 & 29.90 & 30.38 & 30.91 \\
\hline 32 & 26.79 & 26.76 & 26.76 & 26.81 & 26.90 & 27.02 & 27.18 & 27.39 & 27.63 & 27.91 & 28.23 & 28.60 & 29.00 & 29.45 & 29.94 \\
\hline
\end{tabular}




\section{Continued}

\begin{tabular}{|c|c|c|c|c|c|c|c|c|c|c|c|c|c|c|c|}
\hline 33 & 26.33 & 26.27 & 26.24 & 26.26 & 26.31 & 26.40 & 26.53 & 26.70 & 26.90 & 27.15 & 27.44 & 27.76 & 28.13 & 28.54 & 28.99 \\
\hline 34 & 25.90 & 25.81 & 25.75 & 25.73 & 25.75 & 25.80 & 25.90 & 26.03 & 26.20 & 26.42 & 26.67 & 26.96 & 27.29 & 27.66 & 28.07 \\
\hline 35 & 25.49 & 25.36 & 25.27 & 25.22 & 25.20 & 25.23 & 25.29 & 25.39 & 25.53 & 25.70 & 25.92 & 26.18 & 26.47 & 26.81 & 27.18 \\
\hline 36 & 25.10 & 24.94 & 24.82 & 24.73 & 24.68 & 24.67 & 24.70 & 24.77 & 24.87 & 25.02 & 25.20 & 25.42 & 25.68 & 25.98 & 26.32 \\
\hline 37 & 24.73 & 24.53 & 24.38 & 24.26 & 24.18 & 24.14 & 24.13 & 24.17 & 24.24 & 24.35 & 24.50 & 24.68 & 24.91 & 25.17 & 25.48 \\
\hline 38 & 24.37 & 24.15 & 23.96 & 23.81 & 23.70 & 23.62 & 23.59 & 23.59 & 23.63 & 23.70 & 23.82 & 23.97 & 24.16 & 24.39 & 24.66 \\
\hline 39 & 24.03 & 23.77 & 23.55 & 23.37 & 23.23 & 23.12 & 23.06 & 23.03 & 23.03 & 23.08 & 23.16 & 23.28 & 23.44 & 23.63 & 23.87 \\
\hline 40 & 23.71 & 23.42 & 23.17 & 22.95 & 22.78 & 22.64 & 22.54 & 22.48 & 22.46 & 22.47 & 22.52 & 22.61 & 22.73 & 22.89 & 23.09 \\
\hline 41 & 23.40 & 23.08 & 22.79 & 22.55 & 22.34 & 22.18 & 22.04 & 21.95 & 21.90 & 21.88 & 21.89 & 21.95 & 22.04 & 22.17 & 22.34 \\
\hline 42 & 23.10 & 22.75 & 22.44 & 22.16 & 21.92 & 21.72 & 21.56 & 21.44 & 21.35 & 21.30 & 21.29 & 21.31 & 21.37 & 21.47 & 21.60 \\
\hline 43 & 22.82 & 22.43 & 22.09 & 21.78 & 21.51 & 21.28 & 21.09 & 20.93 & 20.82 & 20.74 & 20.69 & 20.68 & 20.71 & 20.78 & 20.88 \\
\hline 44 & 22.54 & 22.13 & 21.75 & 21.41 & 21.11 & 20.85 & 20.63 & 20.44 & 20.30 & 20.18 & 20.11 & 20.07 & 20.07 & 20.11 & 20.18 \\
\hline 45 & 22.28 & 21.83 & 21.42 & 21.06 & 20.73 & 20.43 & 20.18 & 19.97 & 19.79 & 19.64 & 19.54 & 19.47 & 19.44 & 19.44 & 19.48 \\
\hline 46 & 22.02 & 21.54 & 21.10 & 20.70 & 20.34 & 20.02 & 19.74 & 19.49 & 19.29 & 19.11 & 18.98 & 18.88 & 18.82 & 18.79 & 18.80 \\
\hline 47 & 21.77 & 21.26 & 20.79 & 20.36 & 19.97 & 19.62 & 19.31 & 19.03 & 18.79 & 18.59 & 18.43 & 18.30 & 18.21 & 18.15 & 18.13 \\
\hline 48 & 21.53 & 20.98 & 20.48 & 20.02 & 19.60 & 19.22 & 18.88 & 18.57 & 18.31 & 18.08 & 17.88 & 17.73 & 17.60 & 17.52 & 17.47 \\
\hline 49 & 21.28 & 20.71 & 20.18 & 19.69 & 19.24 & 18.83 & 18.45 & 18.12 & 17.82 & 17.56 & 17.34 & 17.16 & 17.01 & 16.89 & 16.81 \\
\hline 50 & 21.04 & 20.43 & 19.87 & 19.35 & 18.87 & 18.43 & 18.03 & 17.67 & 17.34 & 17.05 & 16.80 & 16.59 & 16.41 & 16.27 & 16.16 \\
\hline 51 & 20.80 & 20.16 & 19.57 & 19.02 & 18.51 & 18.04 & 17.61 & 17.22 & 16.86 & 16.55 & 16.27 & 16.02 & 15.82 & 15.65 & 15.51 \\
\hline 52 & 20.55 & 19.88 & 19.26 & 18.68 & 18.14 & 17.64 & 17.18 & 16.76 & 16.38 & 16.04 & 15.73 & 15.46 & 15.22 & 15.03 & 14.86 \\
\hline 53 & 20.29 & 19.60 & 18.94 & 18.33 & 17.76 & 17.24 & 16.75 & 16.30 & 15.89 & 15.52 & 15.19 & 14.89 & 14.63 & 14.40 & 14.21 \\
\hline 54 & 20.03 & 19.30 & 18.62 & 17.98 & 17.38 & 16.83 & 16.31 & 15.83 & 15.40 & 15.00 & 14.64 & 14.31 & 14.03 & 13.77 & 13.56 \\
\hline 55 & 19.76 & 19.00 & 18.29 & 17.62 & 16.99 & 16.41 & 15.86 & 15.36 & 14.89 & 14.47 & 14.08 & 13.73 & 13.42 & 13.14 & 12.89 \\
\hline 56 & 19.47 & 18.68 & 17.94 & 7.24 & 16.58 & 15.97 & 15.40 & 14.87 & 14.38 & 13.93 & 13.51 & 13.13 & 12.79 & 12.49 & 12.22 \\
\hline 57 & 19.16 & 18.34 & 17.57 & 16.84 & 16.16 & 15.52 & 14.92 & 14.36 & 13.85 & 13.37 & 12.93 & 12.52 & 12.16 & 11.83 & 11.53 \\
\hline 58 & 18.83 & 17.98 & 17.18 & 16.42 & 15.71 & 15.05 & 14.42 & 13.84 & 13.29 & 12.79 & 12.32 & 11.90 & 11.51 & 11.15 & 10.83 \\
\hline 59 & 18.47 & 17.60 & 16.77 & 15.98 & 15.24 & 14.55 & 13.90 & 13.29 & 12.72 & 12.19 & 11.70 & 11.25 & 10.83 & 10.45 & 10.11 \\
\hline 60 & 18.09 & 17.18 & 16.32 & 15.51 & 14.74 & 14.02 & 13.35 & 12.71 & 12.12 & 11.56 & 11.05 & 10.57 & 10.13 & 9.73 & 9.36 \\
\hline 61 & 17.66 & 16.73 & 15.84 & 15.00 & 14.21 & 13.46 & 12.76 & 12.10 & 11.48 & 10.90 & 10.37 & 9.86 & 9.40 & 8.98 & 8.59 \\
\hline 62 & 17.19 & 16.23 & 15.32 & 14.45 & 13.64 & 12.86 & 12.14 & 11.45 & 10.81 & 10.21 & 9.65 & 9.12 & 8.64 & 8.19 & 7.78 \\
\hline 63 & 16.68 & 15.69 & 14.75 & 13.86 & 13.02 & 12.22 & 11.47 & 10.76 & 10.10 & 9.47 & 8.89 & 8.34 & 7.84 & 7.37 & 6.93 \\
\hline 64 & 16.11 & 15.09 & 14.12 & 13.21 & 12.34 & 11.52 & 10.75 & 10.02 & 9.33 & 8.69 & 8.08 & 7.51 & 6.99 & 6.50 & 6.04 \\
\hline 65 & 15.47 & 14.43 & 13.44 & 12.50 & 11.61 & 10.77 & 9.97 & 9.22 & 8.51 & 7.85 & 7.22 & 6.63 & 6.09 & 5.58 & 5.10 \\
\hline
\end{tabular}


Table A6. Minimum daily efficiencies (in percent) for latitudes $\left[0^{\circ}, 65^{\circ}\right]$; slope angles $\left[76^{\circ}, 90^{\circ}\right]$.

\begin{tabular}{|c|c|c|c|c|c|c|c|c|c|c|c|c|c|c|c|}
\hline & 76 & 77 & 78 & 79 & 80 & 81 & 82 & 83 & 84 & 85 & 86 & 87 & 88 & 89 & 90 \\
\hline 0 & 89.60 & 92.63 & 95.82 & 99.17 & 102.71 & 106.44 & 110.37 & 114.52 & 118.91 & 123.55 & 128.46 & 133.66 & 139.19 & 145.05 & 151.29 \\
\hline 1 & 86.47 & 89.36 & 92.40 & 95.59 & 98.96 & 102.51 & 106.25 & 110.20 & 114.36 & 118.77 & 123.42 & 128.36 & 133.58 & 139.13 & 145.02 \\
\hline 2 & 83.47 & 86.22 & 89.12 & 92.16 & 95.37 & 98.75 & 102.31 & 106.06 & 110.02 & 114.21 & 118.63 & 123.30 & 128.25 & 133.50 & 139.07 \\
\hline 3 & 80.58 & 83.21 & 85.97 & 88.87 & 91.93 & 95.15 & 98.54 & 102.11 & 105.88 & 109.85 & 114.05 & 118.49 & 123.18 & 128.15 & 133.42 \\
\hline 4 & 77.80 & 80.31 & 82.95 & 85.72 & 88.63 & 91.70 & 94.93 & 98.33 & 101.91 & 105.69 & 109.68 & 113.89 & 118.35 & 123.06 & 128.05 \\
\hline 5 & 75.14 & 77.53 & 80.05 & 82.69 & 85.47 & 88.39 & 91.47 & 94.71 & 98.12 & 101.71 & 105.51 & 109.51 & 113.74 & 118.21 & 122.94 \\
\hline 6 & 72.57 & 74.86 & 77.26 & 79.78 & 82.43 & 85.22 & 88.15 & 91.24 & 94.48 & 97.91 & 101.51 & 105.32 & 109.34 & 113.58 & 118.07 \\
\hline 7 & 70.11 & 72.29 & 74.58 & 76.99 & 79.51 & 82.17 & 84.97 & 87.91 & 91.00 & 94.26 & 97.70 & 101.32 & 105.14 & 109.17 & 113.42 \\
\hline 8 & 67.74 & 69.82 & 72.00 & 74.30 & 76.71 & 79.25 & 81.92 & 84.72 & 87.67 & 90.77 & 94.04 & 97.49 & 101.12 & 104.95 & 108.99 \\
\hline 9 & 65.45 & 67.44 & 69.53 & 71.72 & 74.02 & 76.44 & 78.98 & 81.66 & 84.47 & 87.43 & 90.54 & 93.82 & 97.28 & 100.92 & 104.76 \\
\hline 10 & 63.26 & 65.15 & 67.14 & 69.23 & 71.43 & 73.74 & 76.17 & 78.72 & 81.40 & 84.22 & 87.19 & 90.31 & 93.60 & 97.07 & 100.72 \\
\hline 11 & 61.14 & 62.95 & 64.85 & 66.85 & 68.94 & 71.15 & 73.46 & 75.90 & 78.45 & 81.14 & 83.97 & 86.95 & 90.08 & 93.38 & 96.86 \\
\hline 12 & 59.10 & 60.83 & 62.64 & 64.55 & 66.55 & 68.65 & 70.86 & 73.18 & 75.62 & 78.19 & 80.88 & 83.72 & 86.71 & 89.85 & 93.16 \\
\hline 13 & 57.14 & 58.78 & 60.51 & 62.33 & 64.24 & 66.25 & 68.36 & 70.57 & 72.90 & 75.35 & 77.92 & 80.63 & 83.47 & 86.46 & 89.62 \\
\hline 14 & 55.24 & 56.81 & 58.46 & 60.20 & 62.02 & 63.94 & 65.95 & 68.07 & 70.29 & 72.62 & 75.08 & 77.65 & 80.37 & 83.22 & 86.22 \\
\hline 15 & 53.42 & 54.92 & 56.49 & 58.14 & 59.88 & 61.71 & 63.63 & 65.65 & 67.77 & 70.00 & 72.34 & 74.80 & 77.39 & 80.11 & 82.97 \\
\hline 16 & 51.66 & 53.09 & 54.59 & 56.16 & 57.82 & 59.57 & 61.40 & 63.33 & 65.35 & 67.48 & 69.71 & 72.06 & 74.52 & 77.12 & 79.84 \\
\hline 17 & 49.96 & 51.32 & 52.75 & 54.26 & 55.84 & 57.50 & 59.25 & 61.09 & 63.02 & 65.05 & 67.18 & 69.42 & 71.77 & 74.25 & 76.85 \\
\hline 18 & 48.33 & 49.62 & 50.98 & 52.41 & 53.92 & 55.51 & 57.18 & 58.93 & 60.77 & 62.71 & 64.74 & 66.88 & 69.13 & 71.49 & 73.97 \\
\hline 19 & 46.74 & 47.98 & 49.27 & 50.64 & 52.08 & 53.59 & 55.18 & 56.85 & 58.61 & 60.46 & 62.40 & 64.44 & 66.58 & 68.83 & 71.20 \\
\hline 20 & 45.22 & 46.39 & 47.62 & 48.92 & 50.29 & 51.74 & 53.25 & 54.85 & 56.53 & 58.29 & 60.14 & 62.09 & 64.13 & 66.28 & 68.54 \\
\hline 21 & 43.75 & 44.86 & 46.03 & 47.27 & 48.57 & 49.95 & 51.39 & 52.92 & 54.52 & 56.20 & 57.97 & 59.82 & 61.77 & 63.82 & 65.98 \\
\hline 22 & 42.33 & 43.38 & 44.50 & 45.67 & 46.91 & 48.22 & 49.60 & 51.05 & 52.58 & 54.18 & 55.87 & 57.64 & 59.50 & 61.46 & 63.51 \\
\hline 23 & 40.95 & 41.95 & 43.01 & 44.13 & 45.31 & 46.56 & 47.87 & 49.25 & 50.70 & 52.23 & 53.84 & 55.53 & 57.31 & 59.18 & 61.14 \\
\hline 24 & 39.63 & 40.57 & 41.58 & 42.64 & 43.76 & 44.95 & 46.19 & 47.51 & 48.90 & 50.35 & 51.89 & 53.50 & 55.20 & 56.98 & 58.85 \\
\hline 25 & 38.34 & 39.24 & 40.19 & 41.20 & 42.26 & 43.3 & 44.58 & 45.83 & 47.15 & 48.54 & 50.00 & 51.54 & 53.16 & 54.86 & 56.64 \\
\hline 26 & 37.10 & 37.95 & 38.85 & 39.80 & 40.82 & 41.88 & 43.01 & 44.21 & 45.46 & 46.79 & 48.18 & 49.65 & 51.19 & 52.81 & 54.51 \\
\hline 27 & 35.91 & 36.70 & 37.55 & 38.46 & 39.42 & 40.43 & 41.50 & 42.64 & 43.83 & 45.09 & 46.42 & 47.82 & 49.29 & 50.83 & 52.46 \\
\hline 28 & 34.75 & 35.50 & 36.30 & 37.15 & 38.06 & 39.02 & 40.04 & 41.12 & 42.25 & 43.45 & 44.72 & 46.05 & 47.45 & 48.92 & 50.47 \\
\hline 29 & 33.62 & 34.33 & 35.09 & 35.89 & 36.75 & 37.66 & 38.63 & 39.65 & 40.73 & 41.87 & 43.07 & 44.34 & 45.67 & 47.08 & 48.56 \\
\hline 30 & 32.54 & 33.20 & 33.91 & 34.67 & 35.48 & 36.34 & 37.25 & 38.22 & 39.25 & 40.33 & 41.48 & 42.68 & 43.95 & 45.29 & 46.70 \\
\hline 31 & 31.49 & 32.11 & 32.77 & 33.49 & 34.25 & 35.06 & 35.93 & 36.84 & 37.82 & 38.85 & 39.93 & 41.08 & 42.29 & 43.56 & 44.91 \\
\hline 32 & 30.47 & 31.05 & 31.67 & 32.34 & 33.06 & 33.82 & 34.64 & 35.51 & 36.43 & 37.40 & 38.44 & 39.53 & 40.68 & 41.89 & 43.17 \\
\hline
\end{tabular}




\section{Continued}

\begin{tabular}{|c|c|c|c|c|c|c|c|c|c|c|c|c|c|c|c|}
\hline 33 & 29.48 & 30.02 & 30.60 & 31.23 & 31.90 & 32.62 & 33.39 & 34.21 & 35.08 & 36.01 & 36.99 & 38.02 & 39.12 & 40.27 & 41.49 \\
\hline 34 & 28.53 & 29.02 & 29.56 & 30.15 & 30.78 & 31.46 & 32.18 & 32.95 & 33.78 & 34.65 & 35.58 & 36.56 & 37.60 & 38.70 & 39.86 \\
\hline 35 & 27.60 & 28.06 & 28.56 & 29.10 & 29.69 & 30.33 & 31.01 & 31.73 & 32.51 & 33.34 & 34.22 & 35.15 & 36.13 & 37.18 & 38.28 \\
\hline 36 & 26.70 & 27.12 & 27.58 & 28.08 & 28.63 & 29.23 & 29.86 & 30.55 & 31.28 & 32.06 & 32.89 & 33.77 & 34.71 & 35.70 & 36.74 \\
\hline 37 & 25.82 & 26.20 & 26.63 & 27.10 & 27.60 & 28.16 & 28.75 & 29.39 & 30.08 & 30.82 & 31.60 & 32.44 & 33.32 & 34.26 & 35.25 \\
\hline 38 & 24.97 & 25.32 & 25.70 & 26.13 & 26.60 & 27.12 & 27.67 & 28.27 & 28.92 & 29.61 & 30.35 & 31.14 & 31.97 & 32.86 & 33.81 \\
\hline 39 & 24.14 & 24.45 & 24.80 & 25.20 & 25.63 & 26.10 & 26.62 & 27.18 & 27.78 & 28.43 & 29.13 & 29.87 & 30.66 & 31.50 & 32.40 \\
\hline 40 & 23.33 & 23.61 & 23.93 & 24.28 & 24.68 & 25.12 & 25.59 & 26.11 & 26.68 & 27.29 & 27.94 & 28.64 & 29.38 & 30.18 & 31.02 \\
\hline 41 & 22.54 & 22.79 & 23.07 & 23.39 & 23.75 & 24.15 & 24.59 & 25.08 & 25.60 & 26.17 & 26.78 & 27.44 & 28.14 & 28.89 & 29.69 \\
\hline 42 & 21.78 & 21.99 & 22.23 & 22.52 & 22.85 & 23.21 & 23.61 & 24.06 & 24.55 & 25.08 & 25.65 & 26.26 & 26.92 & 27.63 & 28.38 \\
\hline 43 & 21.02 & 21.20 & 21.42 & 21.67 & 21.96 & 22.29 & 22.66 & 23.07 & 23.52 & 24.01 & 24.54 & 25.12 & 25.74 & 26.40 & 27.11 \\
\hline 44 & 20.29 & 20.43 & 20.61 & 20.83 & 21.09 & 21.39 & 21.72 & 22.09 & 22.51 & 22.96 & 23.46 & 23.99 & 24.57 & 25.20 & 25.86 \\
\hline 45 & 19.56 & 19.68 & 19.83 & 20.01 & 20.24 & 20.50 & 20.80 & 21.14 & 21.52 & 21.94 & 22.39 & 22.89 & 23.43 & 24.02 & 24.65 \\
\hline 46 & 18.85 & 18.93 & 19.05 & 19.21 & 19.40 & 19.63 & 19.90 & 20.20 & 20.55 & 20.93 & 21.35 & 21.81 & 22.32 & 22.86 & 23.45 \\
\hline 47 & 18.15 & 18.20 & 18.29 & 18.42 & 18.58 & 18.78 & 19.01 & 19.28 & 19.59 & 19.94 & 20.33 & 20.75 & 21.22 & 21.73 & 22.28 \\
\hline 48 & 17.46 & 17.48 & 17.54 & 17.63 & 17.76 & 17.93 & 18.13 & 18.37 & 18.65 & 18.97 & 19.32 & 19.71 & 20.14 & 20.61 & 21.12 \\
\hline 49 & 16.77 & 16.77 & 16.79 & 16.86 & 16.96 & 17.10 & 17.27 & 17.48 & 17.72 & 18.00 & 18.32 & 18.68 & 19.08 & 19.51 & 19.99 \\
\hline 50 & 16.09 & 16.06 & 16.06 & 16.09 & 16.16 & 16.27 & 16.41 & 16.59 & 16.80 & 17.05 & 17.34 & 17.66 & 18.03 & 18.43 & 18.87 \\
\hline 51 & 15.41 & 15.35 & 15.32 & 15.33 & 15.37 & 15.45 & 15.56 & 15.71 & 15.89 & 16.11 & 16.36 & 16.66 & 16.99 & 17.35 & 17.76 \\
\hline 52 & 14.74 & 14.65 & 14.59 & 14.57 & 14.58 & 14.63 & 14.71 & 14.83 & 14.98 & 15.17 & 15.40 & 15.66 & 15.96 & 16.29 & 16.66 \\
\hline 53 & 14.06 & 13.94 & 13.86 & 13.81 & 13.79 & 13.81 & 13.87 & 13.96 & 14.08 & 14.24 & 14.43 & 14.66 & 14.93 & 15.23 & 15.57 \\
\hline 54 & 13.38 & 13.23 & 13.12 & 13.04 & 13.00 & 12.99 & 13.02 & 13.08 & 13.18 & 13.31 & 13.47 & 13.67 & 13.91 & 14.18 & 14.49 \\
\hline 55 & 12.69 & 12.51 & 12.38 & 12.27 & 12.21 & 12.17 & 12.17 & 12.20 & 12.27 & 12.37 & 12.51 & 12.68 & 12.89 & 13.13 & 13.41 \\
\hline 56 & 11.99 & 11.79 & 11.63 & 11.50 & 11.40 & 11.34 & 11.31 & 11.32 & 11.36 & 11.44 & 11.55 & 11.69 & 11.87 & 12.08 & 12.33 \\
\hline 57 & 11.28 & 11.05 & 10.86 & 10.71 & 10.59 & 10.50 & 10.45 & 10.43 & 10.45 & 10.49 & 10.58 & 10.69 & 10.84 & 11.02 & 11.24 \\
\hline 58 & 10.55 & 10.30 & 10.09 & 9.91 & 9.76 & 9.65 & 9.57 & 9.53 & 9.52 & 9.54 & 9.59 & 9.68 & 9.81 & 9.96 & 10.15 \\
\hline 59 & 9.80 & 9.53 & 9.29 & 9.09 & 8.92 & 8.78 & 8.68 & 8.61 & 8.57 & 8.57 & 8.60 & 8.66 & 8.76 & 8.89 & 9.05 \\
\hline 60 & 9.03 & 8.74 & 8.47 & 8.25 & 8.05 & 7.89 & 7.77 & 7.67 & 7.61 & 7.59 & 7.59 & 7.63 & 7.70 & 7.80 & 7.94 \\
\hline 61 & 8.23 & 7.91 & 7.63 & 7.38 & 7.16 & 6.98 & 6.83 & 6.71 & 6.63 & 6.58 & 6.56 & 6.57 & 6.62 & 6.70 & 6.81 \\
\hline 62 & 7.40 & 7.06 & 6.76 & 6.48 & 6.25 & 6.04 & 5.87 & 5.73 & 5.62 & 5.55 & 5.50 & 5.49 & 5.52 & 5.57 & 5.66 \\
\hline 63 & 6.54 & 6.17 & 5.85 & 5.55 & 5.29 & 5.06 & 4.87 & 4.71 & 4.58 & 4.48 & 4.42 & 4.39 & 4.38 & 4.41 & 4.48 \\
\hline 64 & 5.62 & 5.24 & 4.89 & 4.58 & 4.30 & 4.05 & 3.84 & 3.65 & 3.50 & 3.38 & 3.30 & 3.24 & 3.22 & 3.23 & 3.27 \\
\hline 65 & 4.66 & 4.26 & 3.89 & 3.56 & 3.26 & 2.99 & 2.76 & 2.55 & 2.38 & 2.25 & 2.14 & 2.06 & 2.02 & 2.00 & 2.02 \\
\hline
\end{tabular}


Table A7. Maximum daily efficiencies for latitudes $\left[0^{\circ}, 65^{\circ}\right]$; slope angles $\left[0^{\circ}, 15^{\circ}\right]$.

\begin{tabular}{|c|c|c|c|c|c|c|c|c|c|c|c|c|c|c|c|c|}
\hline & 0 & 1 & 2 & 3 & 4 & 5 & 6 & 7 & 8 & 9 & 10 & 11 & 12 & 13 & 14 & 15 \\
\hline 0 & 0.71 & 0.73 & 0.76 & 0.78 & 0.80 & 0.83 & 0.85 & 0.88 & 0.91 & 0.94 & 0.98 & 1.01 & 1.05 & 1.09 & 1.13 & 1.17 \\
\hline 1 & 0.72 & 0.72 & 0.74 & 0.76 & 0.79 & 0.81 & 0.84 & 0.86 & 0.89 & 0.92 & 0.95 & 0.99 & 1.02 & 1.06 & 1.10 & 1.14 \\
\hline 2 & 0.74 & 0.72 & 0.73 & 0.75 & 0.77 & 0.80 & 0.82 & 0.85 & 0.87 & 0.90 & 0.93 & 0.96 & 1.00 & 1.03 & 1.07 & 1.11 \\
\hline 3 & 0.75 & 0.73 & 0.72 & 0.74 & 0.76 & 0.78 & 0.81 & 0.83 & 0.86 & 0.88 & 0.91 & 0.94 & 0.98 & 1.01 & 1.04 & 1.08 \\
\hline 4 & 0.77 & 0.74 & 0.72 & 0.73 & 0.75 & 0.77 & 0.79 & 0.81 & 0.84 & 0.87 & 0.89 & 0.92 & 0.95 & 0.99 & 1.02 & 1.06 \\
\hline 5 & 0.78 & 0.76 & 0.73 & 0.71 & 0.73 & 0.75 & 0.78 & 0.80 & 0.82 & 0.85 & 0.88 & 0.90 & 0.93 & 0.96 & 1.00 & 1.03 \\
\hline 6 & 0.80 & 0.77 & 0.75 & 0.73 & 0.72 & 0.74 & 0.76 & 0.79 & 0.81 & 0.83 & 0.86 & 0.89 & 0.91 & 0.94 & 0.98 & 1.01 \\
\hline 7 & 0.82 & 0.79 & 0.76 & 0.74 & 0.72 & 0.73 & 0.75 & 0.77 & 0.80 & 0.82 & 0.84 & 0.87 & 0.90 & 0.93 & 0.96 & 0.99 \\
\hline 8 & 0.83 & 0.81 & 0.78 & 0.76 & 0.73 & 0.72 & 0.74 & 0.76 & 0.78 & 0.80 & 0.83 & 0.85 & 0.88 & 0.91 & 0.94 & 0.97 \\
\hline 9 & 0.85 & 0.82 & 0.80 & 0.77 & 0.75 & 0.72 & 0.73 & 0.75 & 0.77 & 0.79 & 0.81 & 0.84 & 0.86 & 0.89 & 0.92 & 0.95 \\
\hline 10 & 0.87 & 0.84 & 0.82 & 0.79 & 0.76 & 0.74 & 0.72 & 0.74 & 0.76 & 0.78 & 0.80 & 0.82 & 0.85 & 0.87 & 0.90 & 0.93 \\
\hline 11 & 0.89 & 0.86 & 0.83 & 0.81 & 0.78 & 0.75 & 0.73 & 0.73 & 0.75 & 0.77 & 0.79 & 0.81 & 0.83 & 0.86 & 0.88 & 0.91 \\
\hline 12 & 0.92 & 0.88 & 0.85 & 0.82 & 0.80 & 0.77 & 0.75 & 0.72 & 0.74 & 0.76 & 0.78 & 0.80 & 0.82 & 0.84 & 0.87 & 0.90 \\
\hline 13 & 0.94 & 0.91 & 0.87 & 0.84 & 0.82 & 0.79 & 0.76 & 0.74 & 0.73 & 0.75 & 0.77 & 0.79 & 0.81 & 0.83 & 0.85 & 0.88 \\
\hline 14 & 0.97 & 0.93 & 0.90 & 0.86 & 0.83 & 0.81 & 0.78 & 0.75 & 0.73 & 0.74 & 0.76 & 0.78 & 0.80 & 0.82 & 0.84 & 0.87 \\
\hline 15 & 0.99 & 0.95 & 0.92 & 0.89 & 0.85 & 0.83 & 0.80 & 0.77 & 0.74 & 0.73 & 0.75 & 0.77 & 0.79 & 0.81 & 0.83 & 0.85 \\
\hline 16 & 1.02 & 0.98 & 0.94 & 0.91 & 0.88 & 0.85 & 0.82 & 0.79 & 0.76 & 0.74 & 0.74 & 0.76 & 0.78 & 0.80 & 0.82 & 0.84 \\
\hline 17 & 1.05 & 1.01 & 0.97 & 0.93 & 0.90 & 0.87 & 0.84 & 0.81 & 0.78 & 0.75 & 0.73 & 0.75 & 0.77 & 0.79 & 0.81 & 0.83 \\
\hline 18 & 1.08 & 1.04 & 1.00 & 0.96 & 0.92 & 0.89 & 0.86 & 0.83 & 0.80 & 0.77 & 0.74 & 0.74 & 0.76 & 0.78 & 0.80 & 0.82 \\
\hline 19 & 1.11 & 1.07 & 1.02 & 0.98 & 0.95 & 0.91 & 0.88 & 0.85 & 0.82 & 0.79 & 0.76 & 0.73 & 0.75 & 0.77 & 0.79 & 0.81 \\
\hline 20 & 1.14 & 1.10 & 1.05 & 1.01 & 0.97 & 0.94 & 0.90 & 0.87 & 0.84 & 0.81 & 0.78 & 0.75 & 0.74 & 0.76 & 0.78 & 0.80 \\
\hline 21 & 1.18 & 1.13 & 1.08 & 1.04 & 1.00 & 0.96 & 0.92 & 0.89 & 0.86 & 0.83 & 0.80 & 0.77 & 0.74 & 0.75 & 0.77 & 0.79 \\
\hline 22 & 1.22 & 1.16 & 1.12 & 1.07 & 1.03 & 0.99 & 0.95 & 0.91 & 0.88 & 0.85 & 0.82 & 0.79 & 0.76 & 0.75 & 0.76 & 0.78 \\
\hline 23 & 1.25 & 1.20 & 1.15 & 1.10 & 1.06 & 1.02 & 0.98 & 0.94 & 0.90 & 0.87 & 0.84 & 0.81 & 0.78 & 0.75 & 0.76 & 0.77 \\
\hline 24 & 1.30 & 1.24 & 1.19 & 1.14 & 1.09 & 1.05 & 1.00 & 0.96 & 0.93 & 0.89 & 0.86 & 0.83 & 0.80 & 0.77 & 0.75 & 0.77 \\
\hline 25 & 1.34 & 1.28 & 1.22 & 1.17 & 1.12 & 1.08 & 1.03 & 0.99 & 0.95 & 0.92 & 0.88 & 0.85 & 0.82 & 0.79 & 0.76 & 0.76 \\
\hline 26 & 1.39 & 1.32 & 1.27 & 1.21 & 1.16 & 1.11 & 1.06 & 1.02 & 0.98 & 0.94 & 0.90 & 0.87 & 0.84 & 0.81 & 0.78 & 0.76 \\
\hline 27 & 1.43 & 1.37 & 1.31 & 1.25 & 1.20 & 1.14 & 1.10 & 1.05 & 1.01 & 0.97 & 0.93 & 0.89 & 0.86 & 0.83 & 0.79 & 0.77 \\
\hline 28 & 1.49 & 1.42 & 1.35 & 1.29 & 1.23 & 1.18 & 1.13 & 1.08 & 1.04 & 1.00 & 0.95 & 0.92 & 0.88 & 0.85 & 0.81 & 0.78 \\
\hline 29 & 1.54 & 1.47 & 1.40 & 1.34 & 1.27 & 1.22 & 1.17 & 1.12 & 1.07 & 1.02 & 0.98 & 0.94 & 0.91 & 0.87 & 0.84 & 0.80 \\
\hline 30 & 1.60 & 1.52 & 1.45 & 1.38 & 1.32 & 1.26 & 1.20 & 1.15 & 1.10 & 1.05 & 1.01 & 0.97 & 0.93 & 0.89 & 0.86 & 0.82 \\
\hline 31 & 1.66 & 1.58 & 1.50 & 1.43 & 1.36 & 1.30 & 1.24 & 1.19 & 1.14 & 1.09 & 1.04 & 1.00 & 0.96 & 0.92 & 0.88 & 0.85 \\
\hline 32 & 1.73 & 1.64 & 1.56 & 1.48 & 1.41 & 1.35 & 1.28 & 1.23 & 1.17 & 1.12 & 1.07 & 1.03 & 0.98 & 0.94 & 0.91 & 0.87 \\
\hline
\end{tabular}




\section{Continued}

\begin{tabular}{|c|c|c|c|c|c|c|c|c|c|c|c|c|c|c|c|c|}
\hline 33 & 1.80 & 1.70 & 1.62 & 1.54 & 1.46 & 1.39 & 1.33 & 1.27 & 1.21 & 1.16 & 1.11 & 1.06 & 1.01 & 0.97 & 0.93 & 0.89 \\
\hline 34 & 1.87 & 1.77 & 1.68 & 1.60 & 1.52 & 1.44 & 1.37 & 1.31 & 1.25 & 1.19 & 1.14 & 1.09 & 1.04 & 1.00 & 0.96 & 0.92 \\
\hline 35 & 1.95 & 1.84 & 1.75 & 1.66 & 1.57 & 1.50 & 1.42 & 1.36 & 1.29 & 1.23 & 1.18 & 1.12 & 1.07 & 1.03 & 0.98 & 0.94 \\
\hline 36 & 2.03 & 1.92 & 1.82 & 1.72 & 1.63 & 1.55 & 1.47 & 1.40 & 1.34 & 1.27 & 1.21 & 1.16 & 1.11 & 1.06 & 1.01 & 0.97 \\
\hline 37 & 2.13 & 2.01 & 1.89 & 1.79 & 1.70 & 1.61 & 1.53 & 1.45 & 1.38 & 1.32 & 1.26 & 1.20 & 1.14 & 1.09 & 1.04 & 1.00 \\
\hline 38 & 2.22 & 2.09 & 1.98 & 1.87 & 1.77 & 1.67 & 1.59 & 1.51 & 1.43 & 1.36 & 1.30 & 1.24 & 1.18 & 1.13 & 1.08 & 1.03 \\
\hline 39 & 2.33 & 2.19 & 2.06 & 1.95 & 1.84 & 1.74 & 1.65 & 1.56 & 1.48 & 1.41 & 1.34 & 1.28 & 1.22 & 1.16 & 1.11 & 1.06 \\
\hline 40 & 2.44 & 2.29 & 2.16 & 2.03 & 1.92 & 1.81 & 1.71 & 1.62 & 1.54 & 1.46 & 1.39 & 1.32 & 1.26 & 1.20 & 1.14 & 1.09 \\
\hline 41 & 2.57 & 2.40 & 2.26 & 2.12 & 2.00 & 1.89 & 1.78 & 1.69 & 1.60 & 1.51 & 1.44 & 1.37 & 1.30 & 1.24 & 1.18 & 1.13 \\
\hline 42 & 2.70 & 2.52 & 2.36 & 2.22 & 2.09 & 1.97 & 1.85 & 1.75 & 1.66 & 1.57 & 1.49 & 1.42 & 1.35 & 1.28 & 1.22 & 1.16 \\
\hline 43 & 2.84 & 2.65 & 2.48 & 2.32 & 2.18 & 2.05 & 1.93 & 1.82 & 1.72 & 1.63 & 1.55 & 1.47 & 1.39 & 1.32 & 1.26 & 1.20 \\
\hline 44 & 3.00 & 2.79 & 2.60 & 2.44 & 2.28 & 2.14 & 2.02 & 1.90 & 1.79 & 1.69 & 1.60 & 1.52 & 1.44 & 1.37 & 1.30 & 1.24 \\
\hline 45 & 3.18 & 2.95 & 2.74 & 2.56 & 2.39 & 2.24 & 2.11 & 1.98 & 1.87 & 1.76 & 1.67 & 1.58 & 1.49 & 1.42 & 1.34 & 1.28 \\
\hline 46 & 3.37 & 3.11 & 2.89 & 2.69 & 2.51 & 2.35 & 2.20 & 2.07 & 1.95 & 1.83 & 1.73 & 1.64 & 1.55 & 1.47 & 1.39 & 1.32 \\
\hline 47 & 3.58 & 3.30 & 3.05 & 2.83 & 2.64 & 2.46 & 2.30 & 2.16 & 2.03 & 1.91 & 1.80 & 1.70 & 1.61 & 1.52 & 1.44 & 1.37 \\
\hline 48 & 3.81 & 3.50 & 3.23 & 2.99 & 2.77 & 2.58 & 2.41 & 2.26 & 2.12 & 1.99 & 1.87 & 1.77 & 1.67 & 1.58 & 1.49 & 1.41 \\
\hline 49 & 4.07 & 3.72 & 3.42 & 3.16 & 2.92 & 2.72 & 2.53 & 2.36 & 2.21 & 2.08 & 1.95 & 1.84 & 1.73 & 1.63 & 1.54 & 1.46 \\
\hline 50 & 4.36 & 3.97 & 3.64 & 3.34 & 3.09 & 2.86 & 2.66 & 2.48 & 2.31 & 2.17 & 2.03 & 1.91 & 1.80 & 1.70 & 1.60 & 1.51 \\
\hline 51 & 4.69 & 4.25 & 3.88 & 3.55 & 3.27 & 3.02 & 2.80 & 2.60 & 2.42 & 2.27 & 2.12 & 1.99 & 1.87 & 1.76 & 1.66 & 1.57 \\
\hline 52 & 5.07 & 4.57 & 4.14 & 3.78 & 3.46 & 3.19 & 2.95 & 2.73 & 2.54 & 2.37 & 2.22 & 2.07 & 1.95 & 1.83 & 1.72 & 1.63 \\
\hline 53 & 5.50 & 4.92 & 4.44 & 4.03 & 3.68 & 3.38 & 3.11 & 2.88 & 2.67 & 2.48 & 2.32 & 2.16 & 2.03 & 1.90 & 1.79 & 1.69 \\
\hline 54 & 6.01 & 5.33 & 4.78 & 4.31 & 3.92 & 3.58 & 3.29 & 3.03 & 2.80 & 2.60 & 2.42 & 2.26 & 2.11 & 1.98 & 1.86 & 1.75 \\
\hline 55 & 6.60 & 5.80 & 5.16 & 4.63 & 4.19 & 3.81 & 3.48 & 3.20 & 2.95 & 2.73 & 2.54 & 2.36 & 2.20 & 2.06 & 1.93 & 1.81 \\
\hline 56 & 7.30 & 6.35 & 5.60 & 4.99 & 4.49 & 4.06 & 3.70 & 3.38 & 3.11 & 2.87 & 2.66 & 2.47 & 2.30 & 2.15 & 2.01 & 1.88 \\
\hline 57 & 8.15 & 7.00 & 6.11 & 5.40 & 4.82 & 4.34 & 3.93 & 3.59 & 3.29 & 3.02 & 2.79 & 2.59 & 2.40 & 2.24 & 2.09 & 1.96 \\
\hline 58 & 9.20 & 7.78 & 6.71 & 5.88 & 5.21 & 4.66 & 4.20 & 3.81 & 3.48 & 3.19 & 2.93 & 2.71 & 2.51 & 2.34 & 2.18 & 2.03 \\
\hline 59 & 10.53 & 8.74 & 7.43 & 6.43 & 5.65 & 5.01 & 4.49 & 4.05 & 3.68 & 3.36 & 3.09 & 2.84 & 2.63 & 2.44 & 2.27 & 2.11 \\
\hline 60 & 12.27 & 9.92 & 8.29 & 7.08 & 6.15 & 5.42 & 4.82 & 4.33 & 3.91 & 3.56 & 3.25 & 2.99 & 2.75 & 2.55 & 2.36 & 2.20 \\
\hline 61 & 14.63 & 11.45 & 9.35 & 7.86 & 6.74 & 5.88 & 5.19 & 4.63 & 4.16 & 3.77 & 3.43 & 3.14 & 2.89 & 2.66 & 2.47 & 2.29 \\
\hline 62 & 18.03 & 13.48 & 10.69 & 8.80 & 7.44 & 6.42 & 5.61 & 4.97 & 4.44 & 4.00 & 3.63 & 3.31 & 3.03 & 2.79 & 2.57 & 2.38 \\
\hline 63 & 23.35 & 16.31 & 12.43 & 9.98 & 8.28 & 7.04 & 6.10 & 5.35 & 4.75 & 4.25 & 3.84 & 3.49 & 3.18 & 2.92 & 2.68 & 2.48 \\
\hline 64 & 32.84 & 20.53 & 14.79 & 11.47 & 9.31 & 7.78 & 6.66 & 5.79 & 5.10 & 4.53 & 4.07 & 3.68 & 3.34 & 3.05 & 2.80 & 2.58 \\
\hline 65 & 54.58 & 27.49 & 18.16 & 13.44 & 10.58 & 8.68 & 7.31 & 6.28 & 5.48 & 4.84 & 4.32 & 3.89 & 3.52 & 3.20 & 2.93 & 2.69 \\
\hline
\end{tabular}


Table A8. Maximum daily efficiencies for latitudes $\left[0^{\circ}, 65^{\circ}\right]$; slope angles $\left[16^{\circ}, 30^{\circ}\right]$.

\begin{tabular}{|c|c|c|c|c|c|c|c|c|c|c|c|c|c|c|c|}
\hline & 16 & 17 & 18 & 19 & 20 & 21 & 22 & 23 & 24 & 25 & 26 & 27 & 28 & 29 & 30 \\
\hline 0 & 1.21 & 1.26 & 1.31 & 1.37 & 1.42 & 1.48 & 1.55 & 1.62 & 1.69 & 1.77 & 1.85 & 1.94 & 2.04 & 2.15 & 2.26 \\
\hline 1 & 1.18 & 1.23 & 1.28 & 1.33 & 1.38 & 1.44 & 1.50 & 1.57 & 1.63 & 1.71 & 1.79 & 1.87 & 1.97 & 2.06 & 2.17 \\
\hline 2 & 1.15 & 1.19 & 1.24 & 1.29 & 1.34 & 1.40 & 1.45 & 1.52 & 1.58 & 1.65 & 1.73 & 1.81 & 1.90 & 1.99 & 2.09 \\
\hline 3 & 1.12 & 1.16 & 1.21 & 1.25 & 1.30 & 1.36 & 1.41 & 1.47 & 1.53 & 1.60 & 1.67 & 1.75 & 1.83 & 1.92 & 2.01 \\
\hline 4 & 1.09 & 1.13 & 1.18 & 1.22 & 1.27 & 1.32 & 1.37 & 1.43 & 1.49 & 1.55 & 1.62 & 1.69 & 1.77 & 1.85 & 1.94 \\
\hline 5 & 1.07 & 1.11 & 1.15 & 1.19 & 1.23 & 1.28 & 1.33 & 1.39 & 1.44 & 1.50 & 1.57 & 1.64 & 1.71 & 1.79 & 1.87 \\
\hline 6 & 1.04 & 1.08 & 1.12 & 1.16 & 1.20 & 1.25 & 1.30 & 1.35 & 1.40 & 1.46 & 1.52 & 1.59 & 1.66 & 1.73 & 1.81 \\
\hline 7 & 1.02 & 1.06 & 1.09 & 1.13 & 1.17 & 1.22 & 1.26 & 1.31 & 1.36 & 1.42 & 1.48 & 1.54 & 1.60 & 1.67 & 1.75 \\
\hline 8 & 1.00 & 1.03 & 1.07 & 1.11 & 1.15 & 1.19 & 1.23 & 1.28 & 1.33 & 1.38 & 1.43 & 1.49 & 1.56 & 1.62 & 1.69 \\
\hline 9 & 0.98 & 1.01 & 1.04 & 1.08 & 1.12 & 1.16 & 1.20 & 1.25 & 1.29 & 1.34 & 1.40 & 1.45 & 1.51 & 1.57 & 1.64 \\
\hline 10 & 0.96 & 0.99 & 1.02 & 1.06 & 1.09 & 1.13 & 1.17 & 1.21 & 1.26 & 1.31 & 1.36 & 1.41 & 1.47 & 1.53 & 1.59 \\
\hline 11 & 0.94 & 0.97 & 1.00 & 1.03 & 1.07 & 1.11 & 1.14 & 1.19 & 1.23 & 1.27 & 1.32 & 1.37 & 1.43 & 1.49 & 1.55 \\
\hline 12 & 0.92 & 0.95 & 0.98 & 1.01 & 1.05 & 1.08 & 1.12 & 1.16 & 1.20 & 1.24 & 1.29 & 1.34 & 1.39 & 1.45 & 1.50 \\
\hline 13 & 0.91 & 0.93 & 0.96 & 0.99 & 1.03 & 1.06 & 1.10 & 1.13 & 1.17 & 1.21 & 1.26 & 1.31 & 1.36 & 1.41 & 1.46 \\
\hline 14 & 0.89 & 0.92 & 0.95 & 0.97 & 1.01 & 1.04 & 1.07 & 1.11 & 1.15 & 1.19 & 1.23 & 1.27 & 1.32 & 1.37 & 1.42 \\
\hline 15 & 0.88 & 0.90 & 0.93 & 0.96 & 0.99 & 1.02 & 1.05 & 1.09 & 1.12 & 1.16 & 1.20 & 1.24 & 1.29 & 1.34 & 1.39 \\
\hline 16 & 0.86 & 0.89 & 0.91 & 0.94 & 0.97 & 1.00 & 1.03 & 1.06 & 1.10 & 1.14 & 1.18 & 1.22 & 1.26 & 1.31 & 1.35 \\
\hline 17 & 0.85 & 0.87 & 0.90 & 0.93 & 0.95 & 0.98 & 1.01 & 1.04 & 1.08 & 1.11 & 1.15 & 1.19 & 1.23 & 1.28 & 1.32 \\
\hline 18 & 0.84 & 0.86 & 0.89 & 0.91 & 0.94 & 0.97 & 0.99 & 1.03 & 1.06 & 1.09 & 1.13 & 1.17 & 1.21 & 1.25 & 1.29 \\
\hline 19 & 0.83 & 0.85 & 0.87 & 0.90 & 0.92 & 0.95 & 0.98 & 1.01 & 1.04 & 1.07 & 1.11 & 1.14 & 1.18 & 1.22 & 1.26 \\
\hline 20 & 0.82 & 0.84 & 0.86 & 0.88 & 0.91 & 0.93 & 0.96 & 0.99 & 1.02 & 1.05 & 1.09 & 1.12 & 1.16 & 1.20 & 1.24 \\
\hline 21 & 0.81 & 0.83 & 0.85 & 0.87 & 0.90 & 0.92 & 0.95 & 0.98 & 1.00 & 1.03 & 1.07 & 1.10 & 1.14 & 1.17 & 1.21 \\
\hline 22 & 0.80 & 0.82 & 0.84 & 0.86 & 0.88 & 0.91 & 0.93 & 0.96 & 0.99 & 1.02 & 1.05 & 1.08 & 1.12 & 1.15 & 1.19 \\
\hline 23 & 0.79 & 0.81 & 0.83 & 0.85 & 0.87 & 0.90 & 0.92 & 0.95 & 0.97 & 1.00 & 1.03 & 1.06 & 1.10 & 1.13 & 1.17 \\
\hline 24 & 0.78 & 0.80 & 0.82 & 0.84 & 0.86 & 0.89 & 0.91 & 0.93 & 0.96 & 0.99 & 1.02 & 1.05 & 1.08 & 1.11 & 1.15 \\
\hline 25 & 0.78 & 0.80 & 0.81 & 0.83 & 0.86 & 0.88 & 0.90 & 0.92 & 0.95 & 0.98 & 1.00 & 1.03 & 1.06 & 1.10 & 1.13 \\
\hline 26 & 0.77 & 0.79 & 0.81 & 0.83 & 0.85 & 0.87 & 0.89 & 0.91 & 0.94 & 0.96 & 0.99 & 1.02 & 1.05 & 1.08 & 1.11 \\
\hline 27 & 0.77 & 0.78 & 0.80 & 0.82 & 0.84 & 0.86 & 0.88 & 0.90 & 0.93 & 0.95 & 0.98 & 1.00 & 1.03 & 1.06 & 1.10 \\
\hline 28 & 0.76 & 0.78 & 0.80 & 0.81 & 0.83 & 0.85 & 0.87 & 0.89 & 0.92 & 0.94 & 0.97 & 0.99 & 1.02 & 1.05 & 1.08 \\
\hline 29 & 0.77 & 0.78 & 0.79 & 0.81 & 0.83 & 0.85 & 0.87 & 0.89 & 0.91 & 0.93 & 0.96 & 0.98 & 1.01 & 1.04 & 1.07 \\
\hline 30 & 0.79 & 0.77 & 0.79 & 0.81 & 0.82 & 0.84 & 0.86 & 0.88 & 0.90 & 0.92 & 0.95 & 0.97 & 1.00 & 1.02 & 1.05 \\
\hline 31 & 0.81 & 0.78 & 0.79 & 0.80 & 0.82 & 0.84 & 0.85 & 0.87 & 0.89 & 0.92 & 0.94 & 0.96 & 0.99 & 1.01 & 1.04 \\
\hline 32 & 0.83 & 0.80 & 0.78 & 0.80 & 0.82 & 0.83 & 0.85 & 0.87 & 0.89 & 0.91 & 0.93 & 0.96 & 0.98 & 1.01 & 1.03 \\
\hline
\end{tabular}




\section{Continued}

\begin{tabular}{|c|c|c|c|c|c|c|c|c|c|c|c|c|c|c|c|}
\hline 33 & 0.86 & 0.82 & 0.79 & 0.80 & 0.81 & 0.83 & 0.85 & 0.87 & 0.88 & 0.90 & 0.93 & 0.95 & 0.97 & 1.00 & 1.02 \\
\hline 34 & 0.88 & 0.84 & 0.81 & 0.80 & 0.81 & 0.83 & 0.84 & 0.86 & 0.88 & 0.90 & 0.92 & 0.94 & 0.97 & 0.99 & 1.01 \\
\hline 35 & 0.90 & 0.87 & 0.83 & 0.80 & 0.81 & 0.83 & 0.84 & 0.86 & 0.88 & 0.90 & 0.92 & 0.94 & 0.96 & 0.98 & 1.01 \\
\hline 36 & 0.93 & 0.89 & 0.85 & 0.82 & 0.81 & 0.83 & 0.84 & 0.86 & 0.88 & 0.89 & 0.91 & 0.93 & 0.96 & 0.98 & 1.00 \\
\hline 37 & 0.96 & 0.92 & 0.88 & 0.84 & 0.81 & 0.83 & 0.84 & 0.86 & 0.88 & 0.89 & 0.91 & 0.93 & 0.95 & 0.97 & 1.00 \\
\hline 38 & 0.98 & 0.94 & 0.90 & 0.86 & 0.83 & 0.83 & 0.84 & 0.86 & 0.88 & 0.89 & 0.91 & 0.93 & 0.95 & 0.97 & 0.99 \\
\hline 39 & 1.01 & 0.97 & 0.93 & 0.89 & 0.85 & 0.83 & 0.84 & 0.86 & 0.88 & 0.89 & 0.91 & 0.93 & 0.95 & 0.97 & 0.99 \\
\hline 40 & 1.04 & 1.00 & 0.95 & 0.91 & 0.87 & 0.84 & 0.85 & 0.86 & 0.88 & 0.89 & 0.91 & 0.93 & 0.95 & 0.97 & 0.99 \\
\hline 41 & 1.07 & 1.03 & 0.98 & 0.94 & 0.90 & 0.86 & 0.85 & 0.87 & 0.88 & 0.90 & 0.91 & 0.93 & 0.95 & 0.97 & 0.99 \\
\hline 42 & 1.11 & 1.06 & 1.01 & 0.96 & 0.92 & 0.88 & 0.86 & 0.87 & 0.89 & 0.90 & 0.92 & 0.94 & 0.95 & 0.97 & 0.99 \\
\hline 43 & 1.14 & 1.09 & 1.04 & 0.99 & 0.95 & 0.91 & 0.87 & 0.88 & 0.89 & 0.91 & 0.92 & 0.94 & 0.96 & 0.98 & 1.00 \\
\hline 44 & 1.18 & 1.12 & 1.07 & 1.02 & 0.97 & 0.93 & 0.89 & 0.88 & 0.90 & 0.91 & 0.93 & 0.95 & 0.96 & 0.98 & 1.00 \\
\hline 45 & 1.22 & 1.16 & 1.10 & 1.05 & 1.00 & 0.96 & 0.91 & 0.89 & 0.91 & 0.92 & 0.94 & 0.95 & 0.97 & 0.99 & 1.01 \\
\hline 46 & 1.26 & 1.19 & 1.14 & 1.08 & 1.03 & 0.98 & 0.94 & 0.90 & 0.91 & 0.93 & 0.94 & 0.96 & 0.98 & 1.00 & 1.01 \\
\hline 47 & 1.30 & 1.23 & 1.17 & 1.11 & 1.06 & 1.01 & 0.96 & 0.92 & 0.93 & 0.94 & 0.95 & 0.97 & 0.99 & 1.00 & 1.02 \\
\hline 48 & 1.34 & 1.27 & 1.21 & 1.15 & 1.09 & 1.04 & 0.99 & 0.95 & 0.94 & 0.95 & 0.97 & 0.98 & 1.00 & 1.02 & 1.03 \\
\hline 49 & 1.39 & 1.31 & 1.25 & 1.18 & 1.13 & 1.07 & 1.02 & 0.97 & 0.95 & 0.97 & 0.98 & 1.00 & 1.01 & 1.03 & 1.05 \\
\hline 50 & 1.43 & 1.36 & 1.29 & 1.22 & 1.16 & 1.10 & 1.05 & 1.00 & 0.97 & 0.98 & 1.00 & 1.01 & 1.03 & 1.04 & 1.06 \\
\hline 51 & 1.48 & 1.40 & 1.33 & 1.26 & 1.20 & 1.14 & 1.08 & 1.03 & 0.98 & 1.00 & 1.01 & 1.03 & 1.04 & 1.06 & 1.08 \\
\hline 52 & 1.53 & 1.45 & 1.37 & 1.30 & 1.23 & 1.17 & 1.11 & 1.06 & 1.00 & 1.02 & 1.03 & 1.05 & 1.06 & 1.08 & 1.10 \\
\hline 53 & 1.59 & 1.50 & 1.42 & 1.34 & 1.27 & 1.20 & 1.14 & 1.09 & 1.03 & 1.04 & 1.05 & 1.07 & 1.09 & 1.10 & 1.12 \\
\hline 54 & 1.65 & 1.55 & 1.47 & 1.38 & 1.31 & 1.24 & 1.18 & 1.12 & 1.06 & 1.07 & 1.08 & 1.10 & 1.11 & 1.13 & 1.15 \\
\hline 55 & 1.71 & 1.61 & 1.51 & 1.43 & 1.35 & 1.28 & 1.21 & 1.15 & 1.09 & 1.09 & 1.11 & 1.12 & 1.14 & 1.16 & 1.18 \\
\hline 56 & 1.77 & 1.66 & 1.57 & 1.48 & 1.39 & 1.32 & 1.25 & 1.18 & 1.12 & 1.13 & 1.14 & 1.16 & 1.17 & 1.19 & 1.21 \\
\hline 57 & 1.83 & 1.72 & 1.62 & 1.53 & 1.44 & 1.36 & 1.28 & 1.21 & 1.15 & 1.16 & 1.18 & 1.19 & 1.21 & 1.23 & 1.25 \\
\hline 58 & 1.90 & 1.78 & 1.68 & 1.58 & 1.48 & 1.40 & 1.32 & 1.25 & 1.19 & 1.20 & 1.22 & 1.24 & 1.25 & 1.27 & 1.29 \\
\hline 59 & 1.98 & 1.85 & 1.73 & 1.63 & 1.53 & 1.44 & 1.36 & 1.28 & 1.24 & 1.25 & 1.27 & 1.29 & 1.30 & 1.32 & 1.34 \\
\hline 60 & 2.05 & 1.92 & 1.79 & 1.68 & 1.58 & 1.49 & 1.40 & 1.32 & 1.29 & 1.31 & 1.33 & 1.34 & 1.36 & 1.38 & 1.41 \\
\hline 61 & 2.13 & 1.99 & 1.86 & 1.74 & 1.63 & 1.53 & 1.44 & 1.35 & 1.36 & 1.38 & 1.39 & 1.41 & 1.43 & 1.46 & 1.48 \\
\hline 62 & 2.21 & 2.06 & 1.92 & 1.79 & 1.68 & 1.58 & 1.48 & 1.42 & 1.44 & 1.46 & 1.48 & 1.50 & 1.52 & 1.54 & 1.57 \\
\hline 63 & 2.30 & 2.13 & 1.99 & 1.85 & 1.73 & 1.62 & 1.52 & 1.51 & 1.53 & 1.55 & 1.58 & 1.60 & 1.63 & 1.66 & 1.68 \\
\hline 64 & 2.39 & 2.21 & 2.05 & 1.91 & 1.79 & 1.67 & 1.61 & 1.63 & 1.66 & 1.68 & 1.71 & 1.74 & 1.77 & 1.80 & 1.83 \\
\hline 65 & 2.48 & 2.29 & 2.12 & 1.97 & 1.84 & 1.75 & 1.77 & 1.80 & 1.83 & 1.86 & 1.90 & 1.93 & 1.97 & 2.01 & 2.05 \\
\hline
\end{tabular}


Table A9. Maximum daily efficiencies for latitudes $\left[0^{\circ}, 65^{\circ}\right]$; slope angles $\left[31^{\circ}, 45^{\circ}\right]$.

\begin{tabular}{|c|c|c|c|c|c|c|c|c|c|c|c|c|c|c|c|}
\hline & 31 & 32 & 33 & 34 & 35 & 36 & 37 & 38 & 39 & 40 & 41 & 42 & 43 & 44 & 45 \\
\hline 0 & 2.38 & 2.52 & 2.66 & 2.82 & 3.00 & 3.19 & 3.41 & 3.65 & 3.92 & 4.22 & 4.56 & 4.96 & 5.42 & 5.96 & 6.60 \\
\hline 1 & 2.29 & 2.41 & 2.55 & 2.69 & 2.86 & 3.03 & 3.23 & 3.45 & 3.69 & 3.97 & 4.28 & 4.63 & 5.04 & 5.51 & 6.06 \\
\hline 2 & 2.20 & 2.31 & 2.44 & 2.58 & 2.73 & 2.89 & 3.07 & 3.27 & 3.50 & 3.74 & 4.02 & 4.34 & 4.70 & 5.11 & 5.60 \\
\hline 3 & 2.11 & 2.22 & 2.34 & 2.47 & 2.61 & 2.76 & 2.93 & 3.11 & 3.32 & 3.54 & 3.80 & 4.08 & 4.40 & 4.77 & 5.19 \\
\hline 4 & 2.03 & 2.14 & 2.25 & 2.37 & 2.50 & 2.64 & 2.79 & 2.96 & 3.15 & 3.36 & 3.59 & 3.85 & 4.14 & 4.47 & 4.84 \\
\hline 5 & 1.96 & 2.06 & 2.16 & 2.27 & 2.40 & 2.53 & 2.67 & 2.83 & 3.00 & 3.19 & 3.40 & 3.64 & 3.90 & 4.20 & 4.53 \\
\hline 6 & 1.89 & 1.98 & 2.08 & 2.19 & 2.30 & 2.42 & 2.56 & 2.70 & 2.86 & 3.04 & 3.23 & 3.45 & 3.69 & 3.96 & 4.26 \\
\hline 7 & 1.83 & 1.92 & 2.01 & 2.11 & 2.21 & 2.33 & 2.45 & 2.59 & 2.74 & 2.90 & 3.08 & 3.28 & 3.50 & 3.74 & 4.01 \\
\hline 8 & 1.77 & 1.85 & 1.94 & 2.03 & 2.13 & 2.24 & 2.36 & 2.48 & 2.62 & 2.77 & 2.94 & 3.12 & 3.32 & 3.54 & 3.79 \\
\hline 9 & 1.71 & 1.79 & 1.87 & 1.96 & 2.06 & 2.16 & 2.27 & 2.39 & 2.52 & 2.66 & 2.81 & 2.98 & 3.16 & 3.37 & 3.59 \\
\hline 10 & 1.66 & 1.73 & 1.81 & 1.90 & 1.98 & 2.08 & 2.18 & 2.30 & 2.42 & 2.55 & 2.69 & 2.85 & 3.02 & 3.21 & 3.41 \\
\hline 11 & 1.61 & 1.68 & 1.75 & 1.83 & 1.92 & 2.01 & 2.11 & 2.21 & 2.32 & 2.45 & 2.58 & 2.73 & 2.88 & 3.06 & 3.25 \\
\hline 12 & 1.57 & 1.63 & 1.70 & 1.78 & 1.86 & 1.94 & 2.03 & 2.13 & 2.24 & 2.35 & 2.48 & 2.61 & 2.76 & 2.92 & 3.10 \\
\hline 13 & 1.52 & 1.58 & 1.65 & 1.72 & 1.80 & 1.88 & 1.97 & 2.06 & 2.16 & 2.27 & 2.38 & 2.51 & 2.65 & 2.80 & 2.96 \\
\hline 14 & 1.48 & 1.54 & 1.60 & 1.67 & 1.74 & 1.82 & 1.90 & 1.99 & 2.09 & 2.19 & 2.30 & 2.42 & 2.54 & 2.68 & 2.84 \\
\hline 15 & 1.44 & 1.50 & 1.56 & 1.62 & 1.69 & 1.77 & 1.84 & 1.93 & 2.02 & 2.11 & 2.22 & 2.33 & 2.45 & 2.58 & 2.72 \\
\hline 16 & 1.41 & 1.46 & 1.52 & 1.58 & 1.64 & 1.71 & 1.79 & 1.87 & 1.95 & 2.04 & 2.14 & 2.25 & 2.36 & 2.48 & 2.61 \\
\hline 17 & 1.37 & 1.42 & 1.48 & 1.54 & 1.60 & 1.67 & 1.74 & 1.81 & 1.89 & 1.98 & 2.07 & 2.17 & 2.28 & 2.39 & 2.52 \\
\hline 18 & 1.34 & 1.39 & 1.44 & 1.50 & 1.56 & 1.62 & 1.69 & 1.76 & 1.84 & 1.92 & 2.00 & 2.10 & 2.20 & 2.31 & 2.42 \\
\hline 19 & 1.31 & 1.36 & 1.41 & 1.46 & 1.52 & 1.58 & 1.64 & 1.71 & 1.78 & 1.86 & 1.94 & 2.03 & 2.13 & 2.23 & 2.34 \\
\hline 20 & 1.28 & 1.33 & 1.38 & 1.43 & 1.48 & 1.54 & 1.60 & 1.67 & 1.73 & 1.81 & 1.89 & 1.97 & 2.06 & 2.16 & 2.26 \\
\hline 21 & 1.26 & 1.30 & 1.35 & 1.39 & 1.45 & 1.50 & 1.56 & 1.62 & 1.69 & 1.76 & 1.83 & 1.91 & 2.00 & 2.09 & 2.19 \\
\hline 22 & 1.23 & 1.27 & 1.32 & 1.36 & 1.41 & 1.47 & 1.52 & 1.58 & 1.64 & 1.71 & 1.78 & 1.86 & 1.94 & 2.03 & 2.12 \\
\hline 23 & 1.21 & 1.25 & 1.29 & 1.34 & 1.38 & 1.43 & 1.49 & 1.54 & 1.60 & 1.67 & 1.74 & 1.81 & 1.89 & 1.97 & 2.06 \\
\hline 24 & 1.19 & 1.22 & 1.27 & 1.31 & 1.35 & 1.40 & 1.45 & 1.51 & 1.57 & 1.63 & 1.69 & 1.76 & 1.84 & 1.91 & 2.00 \\
\hline 25 & 1.16 & 1.20 & 1.24 & 1.28 & 1.33 & 1.37 & 1.42 & 1.48 & 1.53 & 1.59 & 1.65 & 1.72 & 1.79 & 1.86 & 1.94 \\
\hline 26 & 1.15 & 1.18 & 1.22 & 1.26 & 1.30 & 1.35 & 1.40 & 1.45 & 1.50 & 1.55 & 1.61 & 1.68 & 1.74 & 1.82 & 1.89 \\
\hline 27 & 1.13 & 1.16 & 1.20 & 1.24 & 1.28 & 1.32 & 1.37 & 1.42 & 1.47 & 1.52 & 1.58 & 1.64 & 1.70 & 1.77 & 1.85 \\
\hline 28 & 1.11 & 1.15 & 1.18 & 1.22 & 1.26 & 1.30 & 1.34 & 1.39 & 1.44 & 1.49 & 1.55 & 1.60 & 1.67 & 1.73 & 1.80 \\
\hline 29 & 1.10 & 1.13 & 1.16 & 1.20 & 1.24 & 1.28 & 1.32 & 1.37 & 1.41 & 1.46 & 1.52 & 1.57 & 1.63 & 1.69 & 1.76 \\
\hline 30 & 1.08 & 1.12 & 1.15 & 1.18 & 1.22 & 1.26 & 1.30 & 1.34 & 1.39 & 1.44 & 1.49 & 1.54 & 1.60 & 1.66 & 1.72 \\
\hline 31 & 1.07 & 1.10 & 1.13 & 1.17 & 1.20 & 1.24 & 1.28 & 1.32 & 1.37 & 1.41 & 1.46 & 1.51 & 1.57 & 1.63 & 1.69 \\
\hline 32 & 1.06 & 1.09 & 1.12 & 1.15 & 1.19 & 1.22 & 1.26 & 1.30 & 1.34 & 1.39 & 1.44 & 1.49 & 1.54 & 1.59 & 1.65 \\
\hline
\end{tabular}




\section{Continued}

\begin{tabular}{|c|c|c|c|c|c|c|c|c|c|c|c|c|c|c|c|}
\hline 33 & 1.05 & 1.08 & 1.11 & 1.14 & 1.17 & 1.21 & 1.25 & 1.28 & 1.33 & 1.37 & 1.41 & 1.46 & 1.51 & 1.57 & 1.62 \\
\hline 34 & 1.04 & 1.07 & 1.10 & 1.13 & 1.16 & 1.20 & 1.23 & 1.27 & 1.31 & 1.35 & 1.39 & 1.44 & 1.49 & 1.54 & 1.60 \\
\hline 35 & 1.03 & 1.06 & 1.09 & 1.12 & 1.15 & 1.18 & 1.22 & 1.25 & 1.29 & 1.33 & 1.38 & 1.42 & 1.47 & 1.52 & 1.57 \\
\hline 36 & 1.03 & 1.05 & 1.08 & 1.11 & 1.14 & 1.17 & 1.21 & 1.24 & 1.28 & 1.32 & 1.36 & 1.40 & 1.45 & 1.50 & 1.55 \\
\hline 37 & 1.02 & 1.05 & 1.07 & 1.10 & 1.13 & 1.16 & 1.20 & 1.23 & 1.27 & 1.30 & 1.34 & 1.39 & 1.43 & 1.48 & 1.53 \\
\hline 38 & 1.02 & 1.04 & 1.07 & 1.10 & 1.13 & 1.16 & 1.19 & 1.22 & 1.26 & 1.29 & 1.33 & 1.37 & 1.42 & 1.46 & 1.51 \\
\hline 39 & 1.02 & 1.04 & 1.06 & 1.09 & 1.12 & 1.15 & 1.18 & 1.21 & 1.25 & 1.28 & 1.32 & 1.36 & 1.40 & 1.45 & 1.49 \\
\hline 40 & 1.01 & 1.04 & 1.06 & 1.09 & 1.12 & 1.14 & 1.17 & 1.21 & 1.24 & 1.27 & 1.31 & 1.35 & 1.39 & 1.43 & 1.48 \\
\hline 41 & 1.01 & 1.04 & 1.06 & 1.09 & 1.11 & 1.14 & 1.17 & 1.20 & 1.23 & 1.27 & 1.30 & 1.34 & 1.38 & 1.42 & 1.47 \\
\hline 42 & 1.01 & 1.04 & 1.06 & 1.09 & 1.11 & 1.14 & 1.17 & 1.20 & 1.23 & 1.26 & 1.30 & 1.34 & 1.37 & 1.41 & 1.46 \\
\hline 43 & 1.02 & 1.04 & 1.06 & 1.09 & 1.11 & 1.14 & 1.17 & 1.20 & 1.23 & 1.26 & 1.29 & 1.33 & 1.37 & 1.41 & 1.45 \\
\hline 44 & 1.02 & 1.04 & 1.07 & 1.09 & 1.11 & 1.14 & 1.17 & 1.20 & 1.23 & 1.26 & 1.29 & 1.33 & 1.37 & 1.40 & 1.45 \\
\hline 45 & 1.03 & 1.05 & 1.07 & 1.09 & 1.12 & 1.14 & 1.17 & 1.20 & 1.23 & 1.26 & 1.29 & 1.33 & 1.36 & 1.40 & 1.44 \\
\hline 46 & 1.03 & 1.05 & 1.08 & 1.10 & 1.12 & 1.15 & 1.18 & 1.20 & 1.23 & 1.26 & 1.30 & 1.33 & 1.37 & 1.40 & 1.44 \\
\hline 47 & 1.04 & 1.06 & 1.08 & 1.11 & 1.13 & 1.16 & 1.18 & 1.21 & 1.24 & 1.27 & 1.30 & 1.33 & 1.37 & 1.41 & 1.45 \\
\hline 48 & 1.05 & 1.07 & 1.09 & 1.12 & 1.14 & 1.16 & 1.19 & 1.22 & 1.25 & 1.28 & 1.31 & 1.34 & 1.38 & 1.41 & 1.45 \\
\hline 49 & 1.07 & 1.09 & 1.11 & 1.13 & 1.15 & 1.18 & 1.20 & 1.23 & 1.26 & 1.29 & 1.32 & 1.35 & 1.38 & 1.42 & 1.46 \\
\hline 50 & 1.08 & 1.10 & 1.12 & 1.14 & 1.17 & 1.19 & 1.21 & 1.24 & 1.27 & 1.30 & 1.33 & 1.36 & 1.40 & 1.43 & 1.47 \\
\hline 51 & 1.10 & 1.12 & 1.14 & 1.16 & 1.18 & 1.21 & 1.23 & 1.26 & 1.29 & 1.32 & 1.35 & 1.38 & 1.41 & 1.45 & 1.49 \\
\hline 52 & 1.12 & 1.14 & 1.16 & 1.18 & 1.20 & 1.23 & 1.25 & 1.28 & 1.31 & 1.33 & 1.37 & 1.40 & 1.43 & 1.47 & 1.50 \\
\hline 53 & 1.14 & 1.16 & 1.18 & 1.20 & 1.22 & 1.25 & 1.27 & 1.30 & 1.33 & 1.36 & 1.39 & 1.42 & 1.45 & 1.49 & 1.53 \\
\hline 54 & 1.16 & 1.18 & 1.21 & 1.23 & 1.25 & 1.27 & 1.30 & 1.33 & 1.36 & 1.38 & 1.42 & 1.45 & 1.48 & 1.52 & 1.56 \\
\hline 55 & 1.19 & 1.21 & 1.24 & 1.26 & 1.28 & 1.31 & 1.33 & 1.36 & 1.39 & 1.42 & 1.45 & 1.48 & 1.52 & 1.55 & 1.59 \\
\hline 56 & 1.23 & 1.25 & 1.27 & 1.29 & 1.32 & 1.34 & 1.37 & 1.40 & 1.42 & 1.46 & 1.49 & 1.52 & 1.56 & 1.59 & 1.63 \\
\hline 57 & 1.27 & 1.29 & 1.31 & 1.33 & 1.36 & 1.38 & 1.41 & 1.44 & 1.47 & 1.50 & 1.53 & 1.57 & 1.60 & 1.64 & 1.68 \\
\hline 58 & 1.31 & 1.33 & 1.36 & 1.38 & 1.41 & 1.43 & 1.46 & 1.49 & 1.52 & 1.55 & 1.59 & 1.62 & 1.66 & 1.70 & 1.74 \\
\hline 59 & 1.37 & 1.39 & 1.41 & 1.44 & 1.46 & 1.49 & 1.52 & 1.55 & 1.58 & 1.62 & 1.65 & 1.69 & 1.73 & 1.77 & 1.81 \\
\hline 60 & 1.43 & 1.45 & 1.48 & 1.50 & 1.53 & 1.56 & 1.59 & 1.62 & 1.66 & 1.69 & 1.73 & 1.77 & 1.81 & 1.86 & 1.90 \\
\hline 61 & 1.50 & 1.53 & 1.56 & 1.59 & 1.62 & 1.65 & 1.68 & 1.71 & 1.75 & 1.79 & 1.83 & 1.87 & 1.92 & 1.96 & 2.01 \\
\hline 62 & 1.60 & 1.63 & 1.65 & 1.69 & 1.72 & 1.75 & 1.79 & 1.83 & 1.87 & 1.91 & 1.95 & 2.00 & 2.05 & 2.10 & 2.15 \\
\hline 63 & 1.71 & 1.75 & 1.78 & 1.81 & 1.85 & 1.89 & 1.93 & 1.97 & 2.02 & 2.06 & 2.11 & 2.16 & 2.22 & 2.28 & 2.34 \\
\hline 64 & 1.87 & 1.90 & 1.94 & 1.98 & 2.03 & 2.07 & 2.12 & 2.17 & 2.22 & 2.27 & 2.33 & 2.39 & 2.45 & 2.52 & 2.59 \\
\hline 65 & 2.09 & 2.14 & 2.18 & 2.23 & 2.29 & 2.34 & 2.40 & 2.46 & 2.52 & 2.59 & 2.66 & 2.74 & 2.82 & 2.90 & 2.99 \\
\hline
\end{tabular}


Table A10. Maximum daily efficiencies for latitudes $\left[0^{\circ}, 65^{\circ}\right]$; slope angles $\left[46^{\circ}, 60^{\circ}\right]$.

\begin{tabular}{|c|c|c|c|c|c|c|c|c|c|c|c|c|c|c|c|}
\hline & 46 & 47 & 48 & 49 & 50 & 51 & 52 & 53 & 54 & 55 & 56 & 57 & 58 & 59 & 60 \\
\hline 0 & 7.37 & 8.32 & 9.52 & 11.07 & 13.17 & 16.16 & 20.75 & 28.70 & 45.85 & 109.93 & $23,736.88$ & 3082.76 & 3438.41 & 3931.16 & 1442.46 \\
\hline 1 & 6.72 & 7.51 & 8.49 & 9.73 & 11.35 & 13.54 & 16.69 & 21.60 & 30.27 & 49.79 & 134.17 & 4780.64 & $12,660.18$ & 6690.16 & 2315.88 \\
\hline 2 & 6.16 & 6.84 & 7.66 & 8.67 & 9.95 & 11.64 & 13.94 & 17.27 & 22.52 & 32.03 & 54.47 & 172.11 & 8046.00 & 2437.19 & $25,225.07$ \\
\hline 3 & 5.69 & 6.27 & 6.96 & 7.81 & 8.85 & 10.19 & 11.94 & 14.36 & 17.89 & 23.53 & 34.01 & 60.12 & 239.97 & 4683.88 & 5907.59 \\
\hline 4 & 5.28 & 5.78 & 6.38 & 7.09 & 7.96 & 9.05 & 10.43 & 12.26 & 14.80 & 18.55 & 24.63 & 36.24 & 67.09 & 396.44 & $16,404.51$ \\
\hline 5 & 4.92 & 5.36 & 5.88 & 6.49 & 7.23 & 8.12 & 9.25 & 10.69 & 12.60 & 15.27 & 19.26 & 25.85 & 38.79 & 75.90 & 1142.37 \\
\hline 6 & 4.60 & 4.99 & 5.45 & 5.98 & 6.61 & 7.37 & 8.29 & 9.46 & 10.95 & 12.96 & 15.78 & 20.03 & 27.19 & 41.74 & 87.39 \\
\hline 7 & 4.32 & 4.67 & 5.07 & 5.54 & 6.09 & 6.73 & 7.51 & 8.47 & 9.68 & 11.24 & 13.34 & 16.32 & 20.87 & 28.68 & 45.17 \\
\hline 8 & 4.07 & 4.39 & 4.74 & 5.16 & 5.63 & 6.19 & 6.86 & 7.66 & 8.66 & 9.91 & 11.54 & 13.74 & 16.90 & 21.78 & 30.35 \\
\hline 9 & 3.85 & 4.13 & 4.45 & 4.82 & 5.24 & 5.73 & 6.31 & 6.99 & 7.82 & 8.85 & 10.15 & 11.85 & 14.18 & 17.53 & 22.79 \\
\hline 10 & 3.64 & 3.90 & 4.19 & 4.52 & 4.90 & 5.33 & 5.83 & 6.42 & 7.13 & 7.99 & 9.05 & 10.41 & 12.19 & 14.64 & 18.20 \\
\hline 11 & 3.46 & 3.70 & 3.96 & 4.26 & 4.59 & 4.98 & 5.42 & 5.94 & 6.55 & 7.28 & 8.16 & 9.27 & 10.68 & 12.55 & 15.13 \\
\hline 12 & 3.29 & 3.51 & 3.75 & 4.02 & 4.32 & 4.67 & 5.06 & 5.52 & 6.05 & 6.67 & 7.43 & 8.35 & 9.49 & 10.97 & 12.93 \\
\hline 13 & 3.14 & 3.34 & 3.56 & 3.81 & 4.08 & 4.39 & 4.75 & 5.15 & 5.62 & 6.16 & 6.81 & 7.58 & 8.54 & 9.73 & 11.27 \\
\hline 14 & 3.00 & 3.19 & 3.39 & 3.62 & 3.87 & 4.15 & 4.46 & 4.83 & 5.24 & 5.72 & 6.28 & 6.95 & 7.75 & 8.74 & 9.98 \\
\hline 15 & 2.88 & 3.05 & 3.23 & 3.44 & 3.67 & 3.93 & 4.21 & 4.54 & 4.91 & 5.33 & 5.83 & 6.40 & 7.09 & 7.93 & 8.95 \\
\hline 16 & 2.76 & 2.92 & 3.09 & 3.28 & 3.49 & 3.73 & 3.99 & 4.28 & 4.62 & 5.00 & 5.43 & 5.94 & 6.54 & 7.25 & 8.11 \\
\hline 17 & 2.65 & 2.80 & 2.96 & 3.14 & 3.33 & 3.55 & 3.79 & 4.05 & 4.36 & 4.70 & 5.09 & 5.53 & 6.06 & 6.67 & 7.41 \\
\hline 18 & 2.55 & 2.69 & 2.84 & 3.00 & 3.18 & 3.38 & 3.60 & 3.85 & 4.12 & 4.43 & 4.78 & 5.18 & 5.64 & 6.18 & 6.82 \\
\hline 19 & 2.46 & 2.59 & 2.73 & 2.88 & 3.05 & 3.23 & 3.44 & 3.66 & 3.91 & 4.19 & 4.51 & 4.87 & 5.28 & 5.75 & 6.31 \\
\hline 20 & 2.37 & 2.50 & 2.63 & 2.77 & 2.93 & 3.10 & 3.29 & 3.49 & 3.72 & 3.98 & 4.27 & 4.59 & 4.96 & 5.38 & 5.87 \\
\hline 21 & 2.29 & 2.41 & 2.53 & 2.67 & 2.81 & 2.97 & 3.15 & 3.34 & 3.55 & 3.79 & 4.05 & 4.34 & 4.68 & 5.06 & 5.49 \\
\hline 22 & 2.22 & 2.33 & 2.45 & 2.57 & 2.71 & 2.86 & 3.02 & 3.20 & 3.39 & 3.61 & 3.85 & 4.12 & 4.42 & 4.77 & 5.16 \\
\hline 23 & 2.15 & 2.25 & 2.36 & 2.48 & 2.61 & 2.75 & 2.90 & 3.07 & 3.25 & 3.45 & 3.67 & 3.92 & 4.20 & 4.51 & 4.86 \\
\hline 24 & 2.09 & 2.19 & 2.29 & 2.40 & 2.52 & 2.65 & 2.80 & 2.95 & 3.12 & 3.31 & 3.51 & 3.74 & 3.99 & 4.28 & 4.60 \\
\hline 25 & 2.03 & 2.12 & 2.22 & 2.33 & 2.44 & 2.56 & 2.70 & 2.84 & 3.00 & 3.18 & 3.37 & 3.58 & 3.81 & 4.07 & 4.36 \\
\hline 26 & 1.97 & 2.06 & 2.16 & 2.26 & 2.36 & 2.48 & 2.61 & 2.74 & 2.89 & 3.05 & 3.23 & 3.43 & 3.64 & 3.88 & 4.15 \\
\hline 27 & 1.92 & 2.01 & 2.10 & 2.19 & 2.29 & 2.40 & 2.52 & 2.65 & 2.79 & 2.94 & 3.11 & 3.29 & 3.49 & 3.71 & 3.96 \\
\hline 28 & 1.88 & 1.95 & 2.04 & 2.13 & 2.23 & 2.33 & 2.44 & 2.57 & 2.70 & 2.84 & 3.00 & 3.17 & 3.35 & 3.56 & 3.79 \\
\hline 29 & 1.83 & 1.91 & 1.99 & 2.07 & 2.17 & 2.27 & 2.37 & 2.49 & 2.61 & 2.75 & 2.89 & 3.05 & 3.23 & 3.42 & 3.63 \\
\hline 30 & 1.79 & 1.86 & 1.94 & 2.02 & 2.11 & 2.21 & 2.31 & 2.42 & 2.53 & 2.66 & 2.80 & 2.95 & 3.11 & 3.29 & 3.49 \\
\hline 31 & 1.75 & 1.82 & 1.90 & 1.97 & 2.06 & 2.15 & 2.25 & 2.35 & 2.46 & 2.58 & 2.71 & 2.85 & 3.01 & 3.18 & 3.36 \\
\hline 32 & 1.72 & 1.78 & 1.85 & 1.93 & 2.01 & 2.10 & 2.19 & 2.29 & 2.39 & 2.51 & 2.63 & 2.77 & 2.91 & 3.07 & 3.24 \\
\hline
\end{tabular}




\section{Continued}

\begin{tabular}{|c|c|c|c|c|c|c|c|c|c|c|c|c|c|c|c|}
\hline 33 & 1.68 & 1.75 & 1.82 & 1.89 & 1.97 & 2.05 & 2.14 & 2.23 & 2.33 & 2.44 & 2.56 & 2.69 & 2.82 & 2.97 & 3.13 \\
\hline 34 & 1.65 & 1.72 & 1.78 & 1.85 & 1.93 & 2.00 & 2.09 & 2.18 & 2.28 & 2.38 & 2.49 & 2.61 & 2.74 & 2.88 & 3.04 \\
\hline 35 & 1.63 & 1.69 & 1.75 & 1.82 & 1.89 & 1.96 & 2.04 & 2.13 & 2.22 & 2.32 & 2.43 & 2.54 & 2.67 & 2.80 & 2.95 \\
\hline 36 & 1.60 & 1.66 & 1.72 & 1.79 & 1.85 & 1.93 & 2.00 & 2.09 & 2.18 & 2.27 & 2.37 & 2.48 & 2.60 & 2.73 & 2.87 \\
\hline 37 & 1.58 & 1.64 & 1.69 & 1.76 & 1.82 & 1.89 & 1.97 & 2.05 & 2.13 & 2.22 & 2.32 & 2.43 & 2.54 & 2.66 & 2.79 \\
\hline 38 & 1.56 & 1.61 & 1.67 & 1.73 & 1.80 & 1.86 & 1.94 & 2.01 & 2.09 & 2.18 & 2.28 & 2.38 & 2.48 & 2.60 & 2.72 \\
\hline 39 & 1.54 & 1.59 & 1.65 & 1.71 & 1.77 & 1.84 & 1.91 & 1.98 & 2.06 & 2.14 & 2.23 & 2.33 & 2.43 & 2.54 & 2.66 \\
\hline 40 & 1.53 & 1.58 & 1.63 & 1.69 & 1.75 & 1.81 & 1.88 & 1.95 & 2.03 & 2.11 & 2.20 & 2.29 & 2.39 & 2.49 & 2.61 \\
\hline 41 & 1.51 & 1.56 & 1.62 & 1.67 & 1.73 & 1.79 & 1.86 & 1.93 & 2.00 & 2.08 & 2.16 & 2.25 & 2.35 & 2.45 & 2.56 \\
\hline 42 & 1.50 & 1.55 & 1.60 & 1.66 & 1.71 & 1.77 & 1.84 & 1.90 & 1.97 & 2.05 & 2.13 & 2.22 & 2.31 & 2.41 & 2.52 \\
\hline 43 & 1.50 & 1.54 & 1.59 & 1.64 & 1.70 & 1.76 & 1.82 & 1.88 & 1.95 & 2.03 & 2.11 & 2.19 & 2.28 & 2.38 & 2.48 \\
\hline 44 & 1.49 & 1.53 & 1.58 & 1.63 & 1.69 & 1.75 & 1.81 & 1.87 & 1.94 & 2.01 & 2.09 & 2.17 & 2.25 & 2.35 & 2.45 \\
\hline 45 & 1.49 & 1.53 & 1.58 & 1.63 & 1.68 & 1.74 & 1.80 & 1.86 & 1.92 & 1.99 & 2.07 & 2.15 & 2.23 & 2.32 & 2.42 \\
\hline 46 & 1.48 & 1.53 & 1.58 & 1.62 & 1.68 & 1.73 & 1.79 & 1.85 & 1.91 & 1.98 & 2.06 & 2.13 & 2.22 & 2.30 & 2.40 \\
\hline 47 & 1.49 & 1.53 & 1.58 & 1.62 & 1.67 & 1.73 & 1.78 & 1.84 & 1.91 & 1.98 & 2.05 & 2.12 & 2.20 & 2.29 & 2.38 \\
\hline 48 & 1.49 & 1.53 & 1.58 & 1.63 & 1.68 & 1.73 & 1.79 & 1.84 & 1.91 & 1.97 & 2.04 & 2.12 & 2.20 & 2.28 & 2.37 \\
\hline 49 & 1.50 & 1.54 & 1.59 & 1.63 & 1.68 & 1.73 & 1.79 & 1.85 & 1.91 & 1.98 & 2.04 & 2.12 & 2.20 & 2.28 & 2.37 \\
\hline 50 & 1.51 & 1.55 & 1.60 & 1.64 & 1.69 & 1.74 & 1.80 & 1.86 & 1.92 & 1.98 & 2.05 & 2.12 & 2.20 & 2.28 & 2.37 \\
\hline 51 & 1.53 & 1.57 & 1.61 & 1.66 & 1.71 & 1.76 & 1.81 & 1.87 & 1.93 & 1.99 & 2.06 & 2.13 & 2.21 & 2.29 & 2.38 \\
\hline 52 & 1.54 & 1.59 & 1.63 & 1.68 & 1.72 & 1.78 & 1.83 & 1.89 & 1.95 & 2.01 & 2.08 & 2.15 & 2.23 & 2.31 & 2.40 \\
\hline 53 & 1.57 & 1.61 & 1.65 & 1.70 & 1.75 & 1.80 & 1.85 & 1.91 & 1.97 & 2.04 & 2.10 & 2.18 & 2.25 & 2.33 & 2.42 \\
\hline 54 & 1.60 & 1.64 & 1.68 & 1.73 & 1.78 & 1.83 & 1.89 & 1.94 & 2.00 & 2.07 & 2.14 & 2.21 & 2.29 & 2.37 & 2.45 \\
\hline 55 & 1.63 & 1.67 & 1.72 & 1.77 & 1.82 & 1.87 & 1.92 & 1.98 & 2.04 & 2.11 & 2.18 & 2.25 & 2.33 & 2.41 & 2.50 \\
\hline 56 & 1.67 & 1.72 & 1.76 & 1.81 & 1.86 & 1.91 & 1.97 & 2.03 & 2.09 & 2.16 & 2.23 & 2.30 & 2.38 & 2.47 & 2.56 \\
\hline 57 & 1.72 & 1.77 & 1.82 & 1.86 & 1.92 & 1.97 & 2.03 & 2.09 & 2.15 & 2.22 & 2.30 & 2.37 & 2.45 & 2.54 & 2.63 \\
\hline 58 & 1.79 & 1.83 & 1.88 & 1.93 & 1.98 & 2.04 & 2.10 & 2.16 & 2.23 & 2.30 & 2.38 & 2.46 & 2.54 & 2.63 & 2.73 \\
\hline 59 & 1.86 & 1.91 & 1.96 & 2.01 & 2.07 & 2.13 & 2.19 & 2.26 & 2.33 & 2.40 & 2.48 & 2.56 & 2.65 & 2.74 & 2.85 \\
\hline 60 & 1.95 & 2.00 & 2.05 & 2.11 & 2.17 & 2.23 & 2.30 & 2.37 & 2.44 & 2.52 & 2.61 & 2.70 & 2.79 & 2.89 & 3.00 \\
\hline 61 & 2.06 & 2.12 & 2.18 & 2.24 & 2.30 & 2.37 & 2.44 & 2.52 & 2.60 & 2.68 & 2.77 & 2.87 & 2.97 & 3.08 & 3.20 \\
\hline 62 & 2.21 & 2.27 & 2.33 & 2.40 & 2.47 & 2.54 & 2.62 & 2.71 & 2.80 & 2.89 & 2.99 & 3.10 & 3.21 & 3.34 & 3.47 \\
\hline 63 & 2.40 & 2.47 & 2.54 & 2.61 & 2.69 & 2.78 & 2.87 & 2.97 & 3.07 & 3.18 & 3.29 & 3.42 & 3.55 & 3.69 & 3.84 \\
\hline 64 & 2.67 & 2.75 & 2.83 & 2.92 & 3.02 & 3.12 & 3.23 & 3.34 & 3.46 & 3.60 & 3.74 & 3.89 & 4.05 & 4.23 & 4.42 \\
\hline 65 & 3.09 & 3.19 & 3.30 & 3.41 & 3.53 & 3.66 & 3.80 & 3.96 & 4.12 & 4.29 & 4.48 & 4.68 & 4.91 & 5.15 & 5.41 \\
\hline
\end{tabular}


Table A11. Maximum daily efficiencies for latitudes $\left[0^{\circ}, 65^{\circ}\right]$; slope angles $\left[61^{\circ}, 75^{\circ}\right]$.

\begin{tabular}{|c|c|c|c|c|c|c|c|c|c|c|c|c|c|c|c|}
\hline & 61 & 62 & 63 & 64 & 65 & 66 & 67 & 68 & 69 & 70 & 71 & 72 & 73 & 74 & 75 \\
\hline 0 & 1147 & 2027 & 1044 & 15,029 & 5112 & 6542 & 915 & 15,020 & 1167 & 775 & 724 & 876 & 1695 & 3872 & 721 \\
\hline 1 & 1900 & 5494 & 1750 & 31,407 & 1171 & 954 & 1581 & 2676 & 1374 & 772 & 1006 & 971 & 719 & 1013 & 10,915 \\
\hline 2 & 1558 & 17,036 & 1848 & 2674 & 977 & 2017 & 2070 & 929 & 2886 & 773 & 1436 & 2226 & 1857 & 1053 & 722 \\
\hline 3 & 25,458 & 1521 & 2265 & 1671 & 1450 & 6906 & 2367 & 2730 & 3398 & 935 & 1572 & 10,442 & 10,421 & 21,559 & 1969 \\
\hline 4 & 4319 & 11,375 & 2411 & 1286 & 6248 & 3425 & 1182 & 1037 & 831 & 2041 & 1217 & 43,977 & 1975 & 1555 & 2236 \\
\hline 5 & 46,851 & 1912 & 9359 & 7071 & 4100 & 1052 & 1293 & 2795 & 21,550 & 2829 & 793 & 9858 & 1461 & 900 & 838 \\
\hline 6 & 219,219 & 10,569 & 4792 & 10,345 & 6174 & 3124 & 5260 & 4123 & 1576 & 1142 & 2005 & 2101 & 1608 & 741 & 916 \\
\hline 7 & 103 & 9194 & 3561 & 12,730 & 17,245 & 2020 & 1106 & 1582 & 1210 & 1659 & 1823 & 902 & 3210 & 764 & 1386 \\
\hline 8 & 49 & 126 & 5374 & 2719 & 2946 & 116,455 & 1229 & 3220 & 1942 & 4525 & 2541 & 2657 & 3947 & 905 & 1713 \\
\hline 9 & 32 & 54 & 161 & 8139 & 70,588 & 1740 & 9760 & 2203 & 3205 & 2969 & 1170 & 1142 & 925 & 1649 & 1458 \\
\hline 10 & 24 & 34 & 60 & 225 & 3210 & 2213 & 1904 & 4484 & 14,971 & 1082 & 1220 & 4492 & 7856 & 6676 & 966 \\
\hline 11 & 19 & 25 & 37 & 68 & 372 & 54,480 & 15,297 & 2694 & 2705 & 2878 & 3626 & 16,757 & 1160 & 878 & 1269 \\
\hline 12 & 16 & 20 & 26 & 40 & 77 & 1102 & 6004 & 1782 & 4149 & 1845 & 1268 & 2501 & 1034 & 4123 & 6266 \\
\hline 13 & 13 & 16 & 21 & 28 & 43 & 91 & 12,175 & 4110 & 8082 & 7461 & 1353 & 1824 & 1169 & 4881 & 1171 \\
\hline 14 & 12 & 14 & 17 & 22 & 30 & 47 & 109 & 12,569 & 4718 & 3348 & 20,197 & 1443 & 11,398 & 6346 & 1377 \\
\hline 15 & 10 & 12 & 14 & 18 & 23 & 32 & 52 & 138 & 8888 & 13,789 & 1819 & 75,144 & 2080 & 2518 & 1769 \\
\hline 16 & 9 & 11 & 12 & 15 & 18 & 24 & 34 & 58 & 188 & 3357 & 5720 & 5052 & 18,114 & 4061 & 1103 \\
\hline 17 & 8 & 9 & 11 & 13 & 15 & 19 & 25 & 37 & 66 & 296 & 4292 & 1886 & 7372 & 12,992 & 98,564 \\
\hline 18 & 8 & 9 & 10 & 11 & 13 & 16 & 20 & 27 & 40 & 76 & 701 & 11,989 & 7546 & 2211 & 12,593 \\
\hline 19 & 7 & 8 & 9 & 10 & 12 & 14 & 17 & 21 & 28 & 43 & 90 & 15,661 & 5202 & 2653 & 1519 \\
\hline 20 & 6 & 7 & 8 & 9 & 10 & 12 & 14 & 17 & 22 & 30 & 48 & 111 & 8274 & 2735 & 4413 \\
\hline 21 & 6 & 7 & 7 & 8 & 9 & 11 & 12 & 15 & 18 & 23 & 33 & 54 & 145 & 13,830 & 2288 \\
\hline 22 & 6 & 6 & 7 & 7 & 8 & 9 & 11 & 13 & 15 & 19 & 25 & 35 & 61 & 211 & 5245 \\
\hline 23 & 5 & 6 & 6 & 7 & 8 & 9 & 10 & 11 & 13 & 16 & 20 & 26 & 39 & 71 & 388 \\
\hline 24 & 5 & 5 & 6 & 6 & 7 & 8 & 9 & 10 & 12 & 14 & 17 & 21 & 28 & 43 & 85 \\
\hline 25 & 5 & 5 & 5 & 6 & 7 & 7 & 8 & 9 & 10 & 12 & 14 & 17 & 22 & 30 & 48 \\
\hline 26 & 4 & 5 & 5 & 6 & 6 & 7 & 7 & 8 & 9 & 11 & 13 & 15 & 18 & 24 & 33 \\
\hline 27 & 4 & 5 & 5 & 5 & 6 & 6 & 7 & 8 & 9 & 10 & 11 & 13 & 16 & 19 & 25 \\
\hline 28 & 4 & 4 & 5 & 5 & 5 & 6 & 6 & 7 & 8 & 9 & 10 & 12 & 14 & 16 & 20 \\
\hline 29 & 4 & 4 & 4 & 5 & 5 & 6 & 6 & 7 & 7 & 8 & 9 & 10 & 12 & 14 & 17 \\
\hline 30 & 4 & 4 & 4 & 5 & 5 & 5 & 6 & 6 & 7 & 8 & 8 & 9 & 11 & 13 & 15 \\
\hline 31 & 4 & 4 & 4 & 4 & 5 & 5 & 5 & 6 & 6 & 7 & 8 & 9 & 10 & 11 & 13 \\
\hline 32 & 3 & 4 & 4 & 4 & 4 & 5 & 5 & 6 & 6 & 7 & 7 & 8 & 9 & 10 & 12 \\
\hline
\end{tabular}




\section{Continued}

\begin{tabular}{|c|c|c|c|c|c|c|c|c|c|c|c|c|c|c|c|}
\hline 33 & 3 & 4 & 4 & 4 & 4 & 5 & 5 & 5 & 6 & 6 & 7 & 7 & 8 & 9 & 11 \\
\hline 34 & 3 & 3 & 4 & 4 & 4 & 4 & 5 & 5 & 5 & 6 & 6 & 7 & 8 & 9 & 10 \\
\hline 35 & 3 & 3 & 3 & 4 & 4 & 4 & 4 & 5 & 5 & 6 & 6 & 7 & 7 & 8 & 9 \\
\hline 36 & 3 & 3 & 3 & 4 & 4 & 4 & 4 & 5 & 5 & 5 & 6 & 6 & 7 & 7 & 8 \\
\hline 37 & 3 & 3 & 3 & 3 & 4 & 4 & 4 & 4 & 5 & 5 & 5 & 6 & 6 & 7 & 8 \\
\hline 38 & 3 & 3 & 3 & 3 & 4 & 4 & 4 & 4 & 5 & 5 & 5 & 6 & 6 & 7 & 7 \\
\hline 39 & 3 & 3 & 3 & 3 & 3 & 4 & 4 & 4 & 4 & 5 & 5 & 5 & 6 & 6 & 7 \\
\hline 40 & 3 & 3 & 3 & 3 & 3 & 4 & 4 & 4 & 4 & 4 & 5 & 5 & 6 & 6 & 7 \\
\hline 41 & 3 & 3 & 3 & 3 & 3 & 3 & 4 & 4 & 4 & 4 & 5 & 5 & 5 & 6 & 6 \\
\hline 42 & 3 & 3 & 3 & 3 & 3 & 3 & 4 & 4 & 4 & 4 & 5 & 5 & 5 & 6 & 6 \\
\hline 43 & 3 & 3 & 3 & 3 & 3 & 3 & 3 & 4 & 4 & 4 & 4 & 5 & 5 & 5 & 6 \\
\hline 44 & 3 & 3 & 3 & 3 & 3 & 3 & 3 & 4 & 4 & 4 & 4 & 5 & 5 & 5 & 6 \\
\hline 45 & 3 & 3 & 3 & 3 & 3 & 3 & 3 & 4 & 4 & 4 & 4 & 4 & 5 & 5 & 5 \\
\hline 46 & 2 & 3 & 3 & 3 & 3 & 3 & 3 & 3 & 4 & 4 & 4 & 4 & 5 & 5 & 5 \\
\hline 47 & 2 & 3 & 3 & 3 & 3 & 3 & 3 & 3 & 4 & 4 & 4 & 4 & 5 & 5 & 5 \\
\hline 48 & 2 & 3 & 3 & 3 & 3 & 3 & 3 & 3 & 4 & 4 & 4 & 4 & 4 & 5 & 5 \\
\hline 49 & 2 & 3 & 3 & 3 & 3 & 3 & 3 & 3 & 4 & 4 & 4 & 4 & 4 & 5 & 5 \\
\hline 50 & 2 & 3 & 3 & 3 & 3 & 3 & 3 & 3 & 4 & 4 & 4 & 4 & 4 & 5 & 5 \\
\hline 51 & 2 & 3 & 3 & 3 & 3 & 3 & 3 & 3 & 4 & 4 & 4 & 4 & 4 & 5 & 5 \\
\hline 52 & 2 & 3 & 3 & 3 & 3 & 3 & 3 & 3 & 4 & 4 & 4 & 4 & 4 & 5 & 5 \\
\hline 53 & 3 & 3 & 3 & 3 & 3 & 3 & 3 & 3 & 4 & 4 & 4 & 4 & 4 & 5 & 5 \\
\hline 54 & 3 & 3 & 3 & 3 & 3 & 3 & 3 & 3 & 4 & 4 & 4 & 4 & 4 & 5 & 5 \\
\hline 55 & 3 & 3 & 3 & 3 & 3 & 3 & 3 & 3 & 4 & 4 & 4 & 4 & 4 & 5 & 5 \\
\hline 56 & 3 & 3 & 3 & 3 & 3 & 3 & 3 & 4 & 4 & 4 & 4 & 4 & 5 & 5 & 5 \\
\hline 57 & 3 & 3 & 3 & 3 & 3 & 3 & 3 & 4 & 4 & 4 & 4 & 4 & 5 & 5 & 5 \\
\hline 58 & 3 & 3 & 3 & 3 & 3 & 3 & 4 & 4 & 4 & 4 & 4 & 5 & 5 & 5 & 6 \\
\hline 59 & 3 & 3 & 3 & 3 & 3 & 4 & 4 & 4 & 4 & 4 & 5 & 5 & 5 & 5 & 6 \\
\hline 60 & 3 & 3 & 3 & 4 & 4 & 4 & 4 & 4 & 4 & 5 & 5 & 5 & 5 & 6 & 6 \\
\hline 61 & 3 & 3 & 4 & 4 & 4 & 4 & 4 & 5 & 5 & 5 & 5 & 6 & 6 & 6 & 7 \\
\hline 62 & 4 & 4 & 4 & 4 & 4 & 4 & 5 & 5 & 5 & 6 & 6 & 6 & 7 & 7 & 8 \\
\hline 63 & 4 & 4 & 4 & 5 & 5 & 5 & 5 & 6 & 6 & 6 & 7 & 7 & 8 & 8 & 9 \\
\hline 64 & 5 & 5 & 5 & 5 & 6 & 6 & 6 & 7 & 7 & 8 & 8 & 9 & 10 & 11 & 12 \\
\hline 65 & 6 & 6 & 6 & 7 & 7 & 8 & 8 & 9 & 10 & 11 & 12 & 13 & 14 & 16 & 19 \\
\hline
\end{tabular}


Table A12. Maximum daily efficiencies for latitudes $\left[0^{\circ}, 65^{\circ}\right]$; slope angles $\left[76^{\circ}, 90^{\circ}\right]$.

\begin{tabular}{|c|c|c|c|c|c|c|c|c|c|c|c|c|c|c|c|}
\hline & 76 & 77 & 78 & 79 & 80 & 81 & 82 & 83 & 84 & 85 & 86 & 87 & 88 & 89 & 90 \\
\hline 0 & 2365 & 861 & 3170 & 602 & 2109 & 1650 & 630 & 1033 & 5628 & 1835 & 825 & 595 & 908 & 1835 & Inf \\
\hline 1 & 924 & 1444 & 1074 & 1948 & 666 & 2962 & 1393 & 596 & 1110 & 7786 & 1725 & 810 & 599 & 912 & 1839 \\
\hline 2 & 2262 & 1290 & 1039 & 1426 & 1405 & 747 & 4977 & 1205 & 601 & 1198 & 12,638 & 1628 & 795 & 604 & 917 \\
\hline 3 & 785 & 1260 & 2136 & 811 & 2126 & 1098 & 852 & 15,630 & 1061 & 634 & 1302 & 33,612 & 1541 & 781 & 608 \\
\hline 4 & 15,263 & 1137 & 872 & 6224 & 665 & 4181 & 901 & 991 & 13,649 & 948 & 671 & 1426 & 50,691 & 1462 & 768 \\
\hline 5 & 1060 & 2644 & 2063 & 666 & 6765 & 687 & 135,471 & 764 & 1186 & 4740 & 856 & 712 & 1577 & 14,420 & 1391 \\
\hline 6 & 876 & 694 & 1213 & 11,271 & 780 & 2185 & 843 & 4438 & 662 & 1476 & 2864 & 781 & 760 & 1763 & 8393 \\
\hline 7 & 2048 & 1684 & 977 & 786 & 3235 & 1074 & 1300 & 1089 & 2178 & 619 & 1957 & 2049 & 717 & 814 & 2001 \\
\hline 8 & 17,482 & 8490 & 22,804 & 1898 & 760 & 1410 & 1729 & 924 & 1544 & 1440 & 705 & 2909 & 1593 & 663 & 876 \\
\hline 9 & 6920 & 1636 & 1374 & 1963 & 35,388 & 1150 & 899 & 4464 & 715 & 2658 & 1074 & 820 & 5696 & 1302 & 616 \\
\hline 10 & 9133 & 1139 & 778 & 745 & 937 & 2111 & 2381 & 659 & 7551 & 665 & 9696 & 855 & 982 & 155,860 & 1100 \\
\hline 11 & 5903 & 1097 & 918 & 1213 & 1108 & 763 & 1020 & 30,935 & 835 & 2034 & 840 & 5819 & 710 & 1223 & 6102 \\
\hline 12 & 1430 & 1423 & 1097 & 2845 & 6749 & 3492 & 1332 & 671 & 2048 & 1263 & 1171 & 1142 & 2227 & 606 & 1627 \\
\hline 13 & 1161 & 4051 & 1032 & 9654 & 2549 & 1874 & 2984 & 5337 & 982 & 1054 & 2605 & 820 & 1795 & 1372 & 699 \\
\hline 14 & 3628 & 2161 & 831 & 11,424 & 1405 & 874 & 818 & 1038 & 2625 & 1976 & 707 & 36,058 & 629 & 4231 & 989 \\
\hline 15 & 2162 & 1197 & 1751 & 3226 & 1246 & 878 & 1170 & 1088 & 760 & 1046 & 95,019 & 819 & 2255 & 801 & 11,433 \\
\hline 16 & 1398 & 2044 & 3157 & 1286 & 1520 & 1117 & 3179 & 10,432 & 4472 & 1465 & 686 & 1877 & 1326 & 1158 & 1138 \\
\hline 17 & 2131 & 39,921 & 1390 & 1231 & 3674 & 1137 & 139,992 & 1926 & 1489 & 2087 & 23,535 & 1140 & 942 & 3543 & 776 \\
\hline 18 & 3477 & 18,836 & 1267 & 3990 & 2777 & 910 & 9608 & 1086 & 737 & 739 & 839 & 1652 & 3441 & 675 & 5132 \\
\hline 19 & 15,781 & 1708 & 1674 & 1904 & 1018 & 1266 & 33,633 & 908 & 1253 & 1964 & 1743 & 1024 & 792 & 3302 & 1056 \\
\hline 20 & 2460 & 33,648 & 1107 & 1220 & 3974 & 259,577 & 2500 & 949 & 2204 & 7638 & 2865 & 4644 & 3931 & 957 & 1105 \\
\hline 21 & 2298 & 5900 & 48,489 & 1615 & 3744 & 952 & 988 & 1291 & 3037 & 1780 & 931 & 819 & 984 & 2094 & 2645 \\
\hline 22 & 2461 & 8873 & 18,475 & 11,531 & 3344 & 3285 & 3260 & 3873 & 2326 & 1286 & 989 & 1513 & 1490 & 973 & 819 \\
\hline 23 & 5860 & 3647 & 1535 & 3767 & 5967 & 26,438 & 1117 & 2002 & 1315 & 1293 & 1611 & 36,203 & 3312 & 4602 & 4750 \\
\hline 24 & 2685 & 2501 & 24,536 & 4919 & 2147 & 3814 & 2561 & 1696 & 1117 & 1840 & 2258 & 1947 & 922 & 780 & 892 \\
\hline 25 & 106 & 8170 & 54,839 & 3407 & 3505 & 1524 & 2707 & 1319 & $1,353,239$ & 11,821 & 2074 & 1261 & 1115 & 2015 & 2157 \\
\hline 26 & 54 & 141 & 31,368 & 2927 & 1902 & 1398 & 1193 & 2048 & 1154 & 1648 & 1346 & 1163 & 2319 & 3719 & 1700 \\
\hline 27 & 36 & 62 & 214 & 10,982 & 2200 & 18,221 & 3679 & 1420 & 1620 & 1971 & 1059 & 1405 & 5926 & 1188 & 869 \\
\hline 28 & 27 & 40 & 74 & 448 & 3229 & 2608 & 1942 & 5941 & 1749 & 1323 & 7288 & 2900 & 8859 & 823 & 2586 \\
\hline 29 & 22 & 29 & 45 & 92 & 22,006 & 3145 & 2464 & 4240 & 1646 & 2251 & 1109 & 4119 & 3753 & 1050 & 5275 \\
\hline 30 & 18 & 23 & 32 & 51 & 121 & 235,491 & 2861 & 1920 & 2151 & 1638 & 3125 & 1067 & 1583 & 1124 & 1737 \\
\hline 31 & 16 & 19 & 25 & 36 & 60 & 181 & 9727 & 18,303 & 1787 & 3855 & 4859 & 5071 & 1223 & 972 & 1301 \\
\hline 32 & 14 & 17 & 21 & 27 & 40 & 72 & 361 & 5395 & 2641 & 3047 & 1761 & 4938 & 13,477 & 1439 & 1302 \\
\hline
\end{tabular}




\section{Continued}

\begin{tabular}{|c|c|c|c|c|c|c|c|c|c|c|c|c|c|c|c|}
\hline 33 & 12 & 14 & 18 & 22 & 30 & 45 & 92 & 89,563 & 5180 & 2365 & 133,504 & 7300 & 1620 & 19,624 & 1750 \\
\hline 34 & 11 & 13 & 15 & 19 & 24 & 33 & 53 & 127 & 22,796 & 10,803 & 4507 & 2489 & 1232 & 1252 & 5545 \\
\hline 35 & 10 & 12 & 14 & 16 & 20 & 26 & 37 & 64 & 208 & 6143 & 4331 & 9815 & 1585 & 8455 & 2471 \\
\hline 36 & 9 & 11 & 12 & 14 & 17 & 22 & 29 & 43 & 80 & 600 & 10,645 & 1985 & 16,323 & 8529 & 1687 \\
\hline 37 & 9 & 10 & 11 & 13 & 15 & 19 & 24 & 32 & 50 & 110 & 10,432 & 9255 & 73,402 & 10,164 & 2081 \\
\hline 38 & 8 & 9 & 10 & 12 & 14 & 16 & 20 & 26 & 37 & 61 & 177 & 8818 & 4361 & 1945 & 4727 \\
\hline 39 & 8 & 8 & 9 & 11 & 12 & 15 & 17 & 22 & 29 & 43 & 79 & 473 & $1,789,923$ & 2958 & 2625 \\
\hline 40 & 7 & 8 & 9 & 10 & 11 & 13 & 16 & 19 & 24 & 33 & 51 & 112 & 459,66 & 4059 & 4592 \\
\hline 41 & 7 & 8 & 8 & 9 & 11 & 12 & 14 & 17 & 21 & 27 & 38 & 64 & 199 & 6405 & 26,894 \\
\hline 42 & 7 & 7 & 8 & 9 & 10 & 11 & 13 & 15 & 18 & 23 & 31 & 46 & 88 & 968 & 7542 \\
\hline 43 & 6 & 7 & 8 & 8 & 9 & 10 & 12 & 14 & 16 & 20 & 26 & 36 & 57 & 141 & 373,279 \\
\hline 44 & 6 & 7 & 7 & 8 & 9 & 10 & 11 & 13 & 15 & 18 & 22 & 30 & 43 & 78 & 377 \\
\hline 45 & 6 & 6 & 7 & 8 & 8 & 9 & 10 & 12 & 14 & 16 & 20 & 25 & 35 & 55 & 123 \\
\hline 46 & 6 & 6 & 7 & 7 & 8 & 9 & 10 & 11 & 13 & 15 & 18 & 22 & 29 & 43 & 76 \\
\hline 47 & 6 & 6 & 6 & 7 & 8 & 9 & 9 & 11 & 12 & 14 & 17 & 20 & 26 & 35 & 56 \\
\hline 48 & 5 & 6 & 6 & 7 & 7 & 8 & 9 & 10 & 12 & 13 & 16 & 19 & 23 & 31 & 45 \\
\hline 49 & 5 & 6 & 6 & 7 & 7 & 8 & 9 & 10 & 11 & 13 & 15 & 17 & 21 & 27 & 38 \\
\hline 50 & 5 & 6 & 6 & 7 & 7 & 8 & 9 & 10 & 11 & 12 & 14 & 16 & 20 & 25 & 34 \\
\hline 51 & 5 & 6 & 6 & 6 & 7 & 8 & 8 & 9 & 10 & 12 & 14 & 16 & 19 & 24 & 31 \\
\hline 52 & 5 & 6 & 6 & 6 & 7 & 8 & 8 & 9 & 10 & 12 & 13 & 15 & 18 & 22 & 29 \\
\hline 53 & 5 & 6 & 6 & 6 & 7 & 8 & 8 & 9 & 10 & 11 & 13 & 15 & 18 & 22 & 28 \\
\hline 54 & 5 & 6 & 6 & 6 & 7 & 8 & 8 & 9 & 10 & 11 & 13 & 15 & 18 & 21 & 27 \\
\hline 55 & 5 & 6 & 6 & 7 & 7 & 8 & 8 & 9 & 10 & 12 & 13 & 15 & 18 & 22 & 27 \\
\hline 56 & 5 & 6 & 6 & 7 & 7 & 8 & 9 & 10 & 11 & 12 & 13 & 16 & 18 & 22 & 28 \\
\hline 57 & 6 & 6 & 6 & 7 & 8 & 8 & 9 & 10 & 11 & 12 & 14 & 16 & 19 & 24 & 30 \\
\hline 58 & 6 & 6 & 7 & 7 & 8 & 9 & 9 & 10 & 12 & 13 & 15 & 17 & 21 & 26 & 34 \\
\hline 59 & 6 & 7 & 7 & 8 & 8 & 9 & 10 & 11 & 13 & 14 & 16 & 19 & 24 & 30 & 41 \\
\hline 60 & 7 & 7 & 8 & 8 & 9 & 10 & 11 & 12 & 14 & 16 & 19 & 23 & 28 & 38 & 56 \\
\hline 61 & 7 & 8 & 9 & 9 & 10 & 11 & 13 & 14 & 16 & 19 & 23 & 29 & 38 & 56 & 106 \\
\hline 62 & 8 & 9 & 10 & 11 & 12 & 13 & 15 & 18 & 21 & 25 & 32 & 44 & 68 & 151 & 7135 \\
\hline 63 & 10 & 11 & 12 & 13 & 15 & 18 & 21 & 25 & 31 & 42 & 63 & 125 & 3445 & 3465 & 4183 \\
\hline 64 & 13 & 15 & 17 & 19 & 23 & 28 & 37 & 51 & 84 & 235 & 4167 & 1858 & 4692 & 2196 & 2169 \\
\hline 65 & 22 & 27 & 33 & 45 & 68 & 136 & 15,967 & 102,916 & 3060 & 1838 & 2304 & 1718 & 11,459 & 27,719 & 2484 \\
\hline
\end{tabular}

